April 1994

INFN PI/AE 94/004

\title{
SEARCH FOR THE TOP QUARK AT CDF STUDYING THE STRUCTURE OF EVENTS WITH ONE LEPTON, A NEUTRINO AND JETS
}

\author{
Marina Cobal \\ INFN, Sezione di Pisa
}

University of Pisa Ph. D. Thesis

Profs. G. Bellettini and H. Grassmann, Supervisors

February 1994

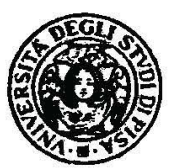

UNIVERSITÃ DEGLI STUDI DI PISA

DIPARTIMENTO DI FISICA

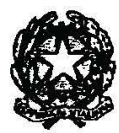

Istituto Nazionale di Fisica Nucleare

Sezione di Pisa 


\title{
UNIVERSITY OF PISA \\ FACULTY OF SCIENCE
}

\author{
Ph.D. Thesis
}

\section{SEARCH FOR THE TOP QUARK AT CDF STUDYING THE STRUCTURE OF EVENTS WITH ONE LEPTON, A NEUTRINO AND JETS}

by Marina Cobal

\author{
Advisor: \\ Prof. G. Bellettini
}

Coordinator:

Prof. P. Menotti

February 1994 


\section{FOREWO RD}

This thesis develops an analysis which looks for the top quark in $p \bar{p} \rightarrow t \bar{t}$ by studying the event structure in the decay channel with one charged lepton, a neutrino and jets. Data collected at the Tevatron by the CDF detector in the 1992-93 run $\left(21.4 p b^{-1}\right)$ were used in this search.

After more than 10 years of planning, construction, calibration and tests, the detector during this run worked in an excellent way. The analysis was done with the supervision of Prof. G. Bellettini and Dr. H. Grassmann, in the context of the heavy flavor working group who periodically discussed the progress. The exchange of information with Dr. S. Leone, who in the same period wrote a thesis on the top search in a different decay channel, was very frequent. 


\section{ACKNOWLEDGMENTS}

First of all I would like to thank Prof. Giorgio Bellettini, who gave me the opportunity to fully dedicate myself to this project. I greatly appreciate his important contribution from the very beginning. Many thanks also for his careful reading of the thesis.

I also would like to thank Dr. Hans Grassmann. This thesis was developed in collaboration with him and completed with the help of his teaching, his ideas, his strenght and enthusiasm.

Thanks to my colleague and friend Sandra Leone. We spent three years working together, exchanging information, discussions, and helping each other. I really hope that we can continue our working relationship also in the future.

Many others helped me during this work. I am particularly grateful to Morris Binkley, Franco Bedeschi and Giovanni Busetto.

I am grateful to Luciana Crovato, Mark Dickson, John Walsh and David Smith for their patient reading of the English translation of my thesis.

But my special thanks go to my parents, who always encouraged and believed in me. I learned from them that one should not take things, not even physics, too seriously. This thesis is dedicated to my father. 


\section{Index}

1 Theoretical Framework $\quad 1$

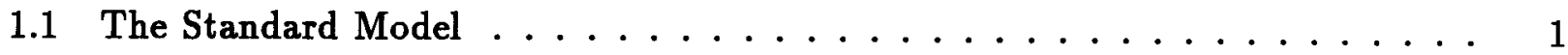

1.2 Parton-Parton Collisions ...................... 4

1.3 The Top Quark .......................... 6

1.3.1 Indirect evidence of the existence of top ............ 6

1.3.2 Experimental hints for the top quark mass . . . . . . . . . . 7

1.3.3 Direct top searches ................... 8

1.3.4 Production and decay of the top quark . . . . . . . . . . . 9

1.4 Exploiting the Single Lepton Event Structure . . . . . . . . . . . . . . . 14

$1.4 .1 \mathrm{~W}+$ jets from $\mathrm{QCD} \ldots \ldots \ldots \ldots \ldots$

1.4.2 Montecarlo simulations . . . . . . . . . . . . . 17

1.4.3 Angular correlation between proton beam and hadronic activity . . . 18

1.4.4 Classification of the event sample . . . . . . . . . . . . . 19

1.4.5 Signal - Background Separation ................ 19

1.4.6 Estimate of the systematic errors . . . . . . . . . . . . 20

2 The Experimental Apparatus $\quad 23$

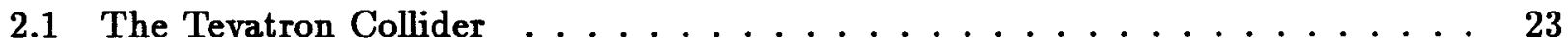

2.2 The CDF Detector . . . . . . . . . . . . . . . . 26

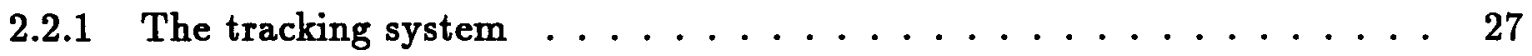

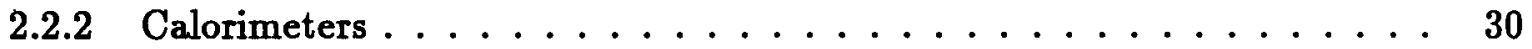

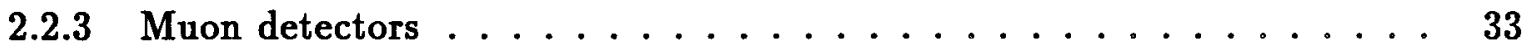


$2.2 .4 \quad$ Trigger $\ldots \ldots \ldots \ldots \ldots \ldots \ldots \ldots \ldots \ldots \ldots \ldots$

3 Event Selection and Reconstruction $\quad 37$

3.1 Electron Identification $\ldots \ldots \ldots \ldots \ldots \ldots \ldots \ldots$

3.2 Muon Identification $\ldots \ldots \ldots \ldots \ldots \ldots \ldots \ldots \ldots$

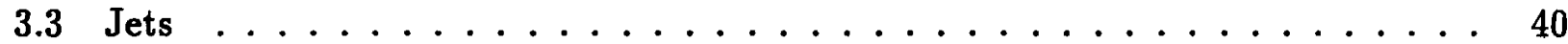

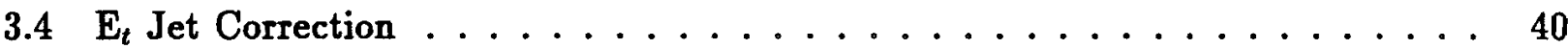

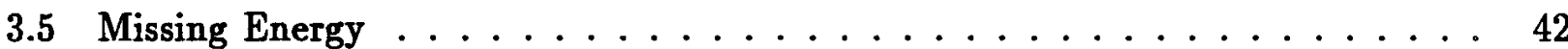

3.6 Missing Energy Correction . . . . . . . . . . . . . . . . . 42

3.7 W Identification . . . . . . . . . . . . . . . . . . . . . . . . . . . 44

4 Montecarlo Simulations $\quad 49$

4.1 VECBOS ......................... 49

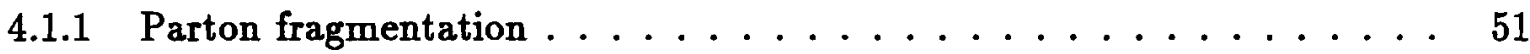

4.1 .2 Reliability of VECBOS + SETPRT $\ldots \ldots \ldots \ldots \ldots \ldots$. . . . . 52

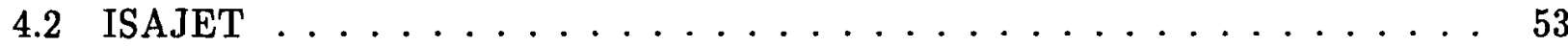

4.3 Detector Simulation . . . . . . . . . . . . 55

5 Search for Top in the CDF Data $\quad 57$

5.1 Selection of $\mathrm{W}+3$ Jet Events . . . . . . . . . . . . . . . 57

5.1 .1 Signal enriched sample $\ldots \ldots \ldots \ldots \ldots \ldots$

5.1 .2 Control sample $\ldots \ldots \ldots \ldots \ldots \ldots \ldots \ldots$

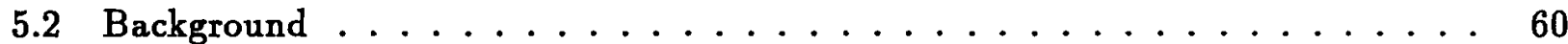

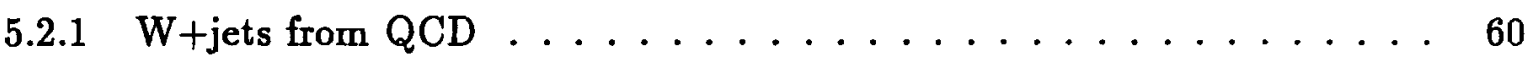

5.2 .2 Non-W background $\ldots \ldots \ldots \ldots \ldots \ldots$

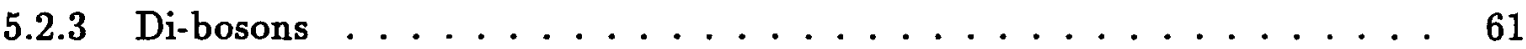

5.3 Choice of Kinematical Variables . . . . . . . . . . . . . . . . 63

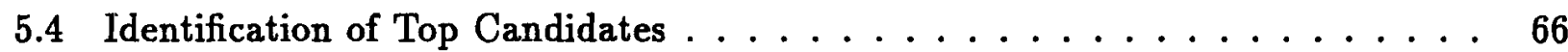

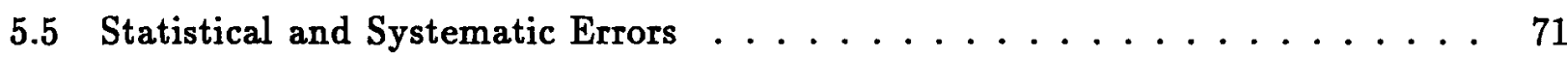

5.5.1 Effects from changing VECBOS parameters on the predictions . . . . 71 
5.5.2 Uncertainty on the ISAJET Montecarlo predictions . . . . . . . 72

5.5.3 Uncertainty in the calorimeter response linearity . . . . . . . . 74

5.5.4 Background distribution in $\ln (\mathrm{rL}) \ldots \ldots \ldots \ldots \ldots$

6 Compatibility with Top Hypothesis $\quad 79$

6.1 Identification of b Quarks in the Selected Events . . . . . . . . . 79

6.2 Four Jet Events . . . . . . . . . . . . . . . . . 85

6.3 Di-lepton Candidates . . . . . . . . . . . . . . . 85

6.4 Reconstruction of the $t \bar{t}$ Mass ...................... 88

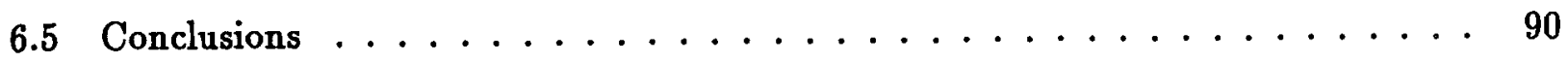

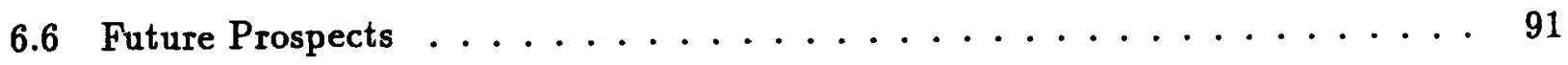

A Effects of the CMX Trigger on W+jet Events 93

$\begin{array}{lr}\text { B Choice of Kinematical Cuts } & 97\end{array}$ 


\section{Chapter 1}

\section{Theoretical Framework}

This thesis is devoted to the top quark search within the Standard Model. Therefore, the theoretical framework within which one expects the top quark to exist and to be important will be briefly explained in the following.

\subsection{The Standard Model}

The Standard Model (SM) is the present theory to describe the elementary particles which constitute our Universe, and the interactions among them. Within the Standard Model there are two kinds of particles: fermions and vector (gauge) bosons. In the "minimal SM" there is also a scalar boson: the Higgs[1], which gives mass to the gauge bosons by the spontaneous symmetry breaking mechanism.

The fermions, pointlike objects of spin $\frac{1}{2}$, are in turn organized in quarks and leptons. There are three kinds (flavors) of quarks, and the quarks exist in three "families" (see table 1.1). Each quark is characterized by its mass, it has fractional electric charge and an additional degree of freedom called "color".

Leptons are the second group of fermions. The electron $(e)$, the muon $(\mu)$ and the tau $(\tau)$ have a non-zero mass and charge 1. Each charged lepton has a corresponding neutrino. The neutrinos have a very small and possibly zero mass, and do not carry electric charge. Measurements performed at LEP confirm the predictions of the SM which requires only 3 light neutrinos[2]. 
All left-handed fermions (spin oriented opposite to momentum) are doublets under the weak isospin group transformations (third component of the weak isospin $\mathrm{T}_{3 L}=\frac{1}{2}$ ). The right handed ones instead are singlets $\left(\mathrm{T}_{3 R}=0\right)$. Table 1.1 summarizes the elementary particle properties. Leaving gravitation aside, there are three different interactions between these particles: weak

\begin{tabular}{|c|c|c|c|c|c|}
\hline Family: & 1 & 2 & 3 & $T_{3}$ & $\bar{Q}$ \\
\hline Leptons: & $\begin{array}{c}\nu_{e} \\
e\end{array}$ & 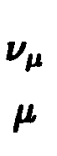 & $\left.\begin{array}{c}\nu_{\tau} ? \\
\tau\end{array}\right)_{L}$ & $\begin{array}{c}1 / 2 \\
-1 / 2\end{array}$ & $\begin{array}{c}0 \\
-1\end{array}$ \\
\hline Quarks: & $\left.\begin{array}{l}u \\
d\end{array}\right)$ & $\begin{array}{l}c \\
s\end{array}$ & $\left(\begin{array}{c}t ? \\
b\end{array}\right)$ & $\begin{array}{c}1 / 2 \\
-1 / 2 \\
\end{array}$ & $\begin{array}{r}2 / 3 \\
-1 / 3 \\
\end{array}$ \\
\hline Higgs: & & $=$ & & $\begin{array}{c}1 / 2 \\
-1 / 2\end{array}$ & $\begin{array}{l}1 \\
0\end{array}$ \\
\hline
\end{tabular}

Table 1.1: Leptons, quarks and the Higgs boson as described in the SM. Also shown are the quantum numbers of $T_{3}$ (third component of the weak isospin) and $Q$ (electric charge).

(for all fermions), electromagnetic (for charged fermions) and strong (for quarks). Two gauge theories describe these interactions: the Electroweak Theory (EW)[3] and Quantum Chromodynamics (QCD[4]). QCD describes the strong interactions and is based on the non-abelian SU(3) group. The Electroweak Theory, which includes Quantum Electrodynamics (QED), describes weak and electromagnetic interactions, and is based on the $\mathrm{SU}(2)_{L} \times \mathrm{U}(1)$ group. The gauge bosons mediate these forces. The photon $(\gamma)$, the $\mathrm{W}$ and the $\mathrm{Z}^{0}$ mediate the electroweak force. Eight coloured gluons carry the strong force between particles with color. Table 1.2 shows some properties of the gauge bosons. With a variety of 6 flavors, the weak interactions are described by unitary transformations between three doublets. These unitary transformations can be represented by a matrix with three Euler angles and six phases (Kobayashi-Maskawa matrix[5]). The existence of the sixth quark, the top, is necessary in the basic structure of the theory. QED is based on the invariance of the interaction under a 


\begin{tabular}{|c|c|r|c|c|}
\hline Bosons & Spin & Charge & Color & Mass $\left(\mathrm{GeV} / \mathrm{c}^{2}\right)$ \\
\hline \hline$\gamma$ & 1 & 0 & no & 0 \\
\hline$Z^{\circ}$ & 1 & 0 & no & $91.187 \pm 0.007[2]$ \\
$W^{ \pm}$ & 1 & \pm 1 & no & $80.22 \pm 0.26[2]$ \\
\hline$g_{i}(i=1, \ldots, 8)$ & 1 & 0 & yes & 0 \\
\hline
\end{tabular}

Table 1.2: The gauge bosons of the SM and their properties.

local, one-dimensional phase rotation (U(1), electric charge). QCD on the contrary is a local, non-abelian gauge theory described by a three-dimensional gauge symmetry ( $\mathrm{SU}(3)$, color) in which the generators do not commute. This difference between QCD and QED manifests itself in the different behavior of their intermediate bosons. In QED photons interact only with fermion-antifermion pairs but not with each other, since a photon does not carry electric charge. In QCD gluons carry color charge and can interact with each other, not only with quarks. These interactions determine the behavior of the strong ("running") coupling constant, as a function of $\mathrm{Q}^{2}$ (the squared four-momentum $\left(\mathrm{q}^{2}\right)$ transferred, with opposite sign). The effective coupling in $\mathrm{QCD}$, at the first order in $1 / \ln \left(Q^{2}\right)$ ("leading $\log$ " or "LO"), is given by:

$$
\alpha_{s}\left(Q^{2}\right)=\frac{1}{\frac{b^{0}}{4 \pi} \ln \frac{Q^{2}}{\Lambda_{Q C D}^{2}}}
$$

$b^{0}$ (defined as $\frac{11}{3} N_{c}-\frac{2}{3} N_{f}$, with $N_{c}=$ number of colors and $N_{f}=$ number of flavors) is a positive number. $\Lambda_{Q C D}^{2}$ is a scale constant whose value is not predicted by the theory, which appears because of the LO approximation. In practice, $\Lambda_{Q C D}$ is a free parameter which needs to be determined by the experiment. $\alpha_{s}\left(Q^{2}\right) \rightarrow \infty$ for $Q^{2} \rightarrow \Lambda_{Q C D}^{2}$. This aspect of the theory corresponds to the experimental observation that free quarks are not found. From expression 1.1.1 one can see that $\alpha_{s} \rightarrow 0$ as $Q^{2} \rightarrow \infty$. In this limit the effective coupling becomes zero, and one reaches the region of the so-called asymptotic freedom. The closer one comes to this region, the better one can use perturbative techniques, like for instance in the calculation of cross sections. 


\subsection{Parton-Parton Collisions}

Hadron colliders allow tests of QCD predictions for many processes, like the production of heavy quarks, including the top quark. In $p \bar{p}$ collisions the proton and the antiproton do not act as pointlike particles, but rather as if they were composed by three ("valence") quarks and by pairs of virtual quarks and gluons. Most frequently the partons undergo interactions at relatively low momentum transfer. Occasionally one can have a high momentum transfer and production of highly energetic states away from the beam, including $t \bar{t}$ states. Final

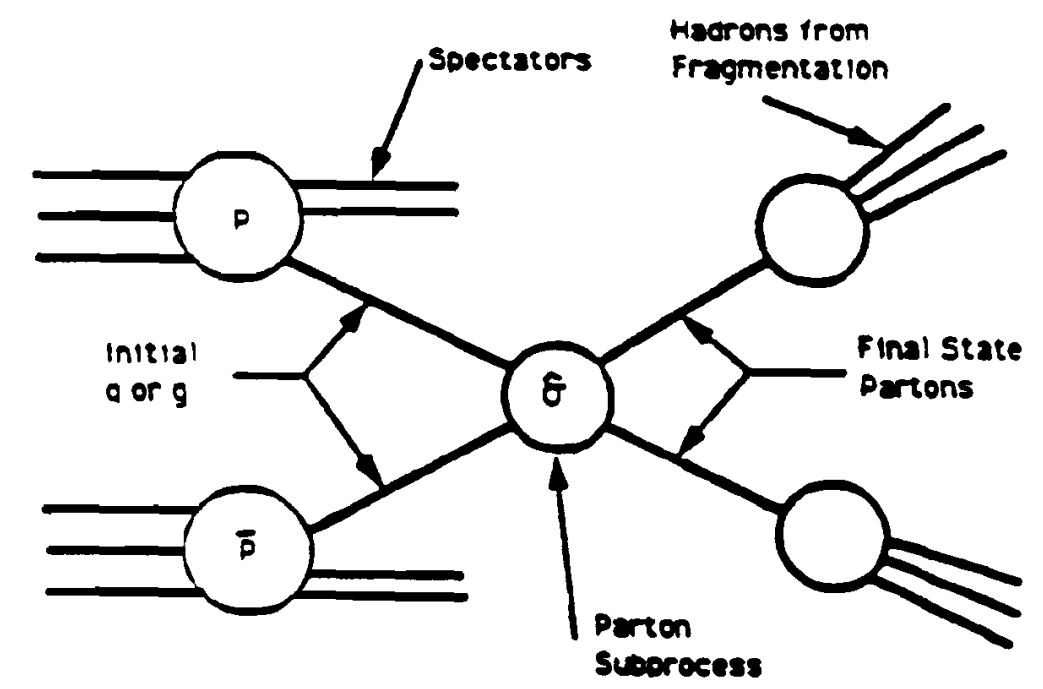

Figure 1.1: Schematic view of a hard scattering process in a $p \bar{p}$ collision.

state gluons and quarks fragment into hadrons approximately collinear with the direction of the parent parton. These particles which emerge from the fragmentation of a parton form a "jet". Partons which do not participate in the hard scattering are called "spectators". Their contribution to the event consists in the production of low-energy particles, which form the so called "underlying event".

In order to calculate the cross section for a process, one needs to convolute the parton cross section with the momentum distribution of the partons inside the $p$ and the $\bar{p}$ :

$$
\sigma=\Sigma_{i, j} \int d x_{i} d x_{j} f\left(x_{i}\right) f\left(x_{j}\right) \tilde{\sigma}\left(p_{i} p_{j}\right)
$$


$\tilde{\sigma}\left(p_{i} p_{j}\right)$ is the cross section with which parton $i$ with momentum $p_{i}$ and parton $j$ with momentum $p_{j}$ create the final state of interest. The sum over all possible partons gives the total cross section $\sigma$ of the process. The function $f\left(x_{i}\right)\left(f\left(x_{j}\right)\right)$ gives the probability for parton $i(j)$ to carry the fraction $x_{i}\left(x_{j}\right)$ of the proton (antiproton) momentum. The $f\left(x_{i}\right)$ functions depend on the kind of parton and are measured primarily in lepton-nucleon scattering experiments. Their evolution at high $Q^{2}$ can be determined by using the Altarelli- Parisi equation[6].

The momentum of two colliding partons is not known a priori. Furthermore the products of the collision which are emitted at small angles can escape detection. For this reason the momentum component of the event parallel to the proton beam cannot be measured and cannot be used to describe the event. One can instead use the transverse component, which is essentially zero for the initial state of the interaction.

Some of the variables which are used in this thesis are :

- $\mathrm{P}_{t}=\mathrm{p} \cdot \sin (\theta)=$ transverse momentum, when $\mathrm{p}=$ momentum and $\theta=$ polar angle measured assuming the beam as $z$ axis and the center of the detector as origin $(z=0)$;

- $\mathrm{E}_{t}=$ transverse energy;

- $\mathrm{M}_{\text {trans }}^{i, j}=\left(\left(\mathrm{P}_{x}^{i}+\mathrm{P}_{x}^{j}\right)^{2}+\left(\mathrm{P}_{y}^{i}+\mathrm{P}_{y}^{j}\right)^{2}\right)^{\frac{1}{2}}$, transverse mass of two particles or two jets, $i$ and $j$, with $x$ and $y$ being two orthogonal components normal to the beam direction. This quantity is invariant under transformations ("boosts") along the beam direction;

- $\eta=-\log \left(\tan \frac{\theta}{2}\right)=$ pseudorapidity.

The large center of mass energy of the hadron colliders (the $S p \bar{p} S$ at CERN and the Tevatron at Fermilab) made it possible to identify processes involving quarks and gluons as those with highly energetic jets. Some of the more recent examples are:

- Study of the transverse energy distribution for multijet events (up to 6 partons in the final state)[7];

- Measurement of the inclusive jet cross section as a function of $P_{t}[8],[9]$; 
- Measurement of the two-jet cross section as a function of their invariant mass[10];

- Study of the jet angular distribution[11];

- Measurement of the $b$ quark production cross section[12].

A measurement precision up to a few $\%$ is achieved and one can do QCD tests which, though less accurate than QED tests, give a good confirmation of the theory.

\subsection{The Top Quark}

The top quark, the "up" element of the third quark doublet, is an essential component of the SM (see table 1.1). Together with the $b$ quark, the $\tau$ lepton and the $\nu_{\tau}$, it completes the third family and completes the symmetry of the theory. This symmetry is necessary to cancel the so called "chiral anomalies". A SM free of anomalies requires that the sum of the charges within a family be 0 . Given the $b$ quark and the $\tau$, there has to be another component of the third family with charge $\frac{2}{3}$.

\subsubsection{Indirect evidence of the existence of top}

There are several indirect evidences for the existence of the top quark and the most important ones are the following:

- Supression of flavor changing neutral currents (GIM mechanism).

The GIM mechanism provides the suppression of flavor changing neutral currents (" $F C N C^{\prime \prime}$ ), if each family has the same singlet + doublet isospin structure. Many experimental evidences show that the flavor changing neutral coupling is very weak. For example, if this coupling would be significant we should observe the $F C N C$ contribution in several processes involving the $B$ meson. The following experimental limits exist :

a) Branching ratio of mesons with $b$ quarks into lepton pairs.

If the $b$ quark were an $\mathrm{SU}(2)_{L}$ singlet, one would expect a branching ratio of the $B$ meson in lepton pairs $>1.3 \times 10^{-2}[14]$. The value predicted by the SM is instead $10^{-4}$ times smaller. 
The CLEO experiment measured that this branching ratio is $<1.2 \times 10^{-3}$ al $90 \%$ C.L.[15] b) $B_{d}^{0}-\bar{B}_{d}^{0}$ oscillations.

. The $B_{d}^{0}-\bar{B}_{d}^{0}$ oscillations are a second order weak process. If the $b$ were a singlet, the $F C N C$ would make the oscillations very likely[16]. The experimentally observed mixing between $B_{d}^{0}$ and $\bar{B}_{d}^{0}[17]$ confirms the existence of a weak isospin partner.

- Forward - backward asymmetry in the process $e^{+} e^{-} \rightarrow b^{+} b^{-}$.

This asymmetry is proportional to [18]:

$$
T_{3 L}^{b}-T_{3 R}^{b}
$$

Were $b$ a singlet (and top would not exist), the asymmetry would be 0 . Data from LEP[19] give a value for $T_{3 L}^{b}-T_{3 R}^{b}$ equal to $-0.504_{-0.011}^{+0.018}$, not consistent with zero and consistent with $T_{3 L}^{b}=-\frac{1}{2}$ e $T_{3 R}^{b}=0$, and with the $b$ being a member of a doublet.

- Decay of the $\mathrm{Z}^{0}$ into $b$ pairs.

If $b$ were a singlet, the partial width of the decay $Z^{0} \rightarrow b \bar{b}\left(\Gamma_{b \bar{b}}\right)$ would need to be about 15 times smaller than observed [20].

\subsubsection{Experimental hints for the top quark mass}

Several lower limits on the top mass $\left(\mathrm{M}_{\text {top }}\right)$ have been given. It is also possible to indirectly give an estimate of $\mathrm{M}_{\text {top }}$.

- Measurement of the $R$ parameter

If we define $R$ as the ratio of the total hadronic cross section and the point-like cross section $\left(e^{+} e^{-} \rightarrow \mu^{+} \mu^{-}\right):$

$$
R=\frac{\sigma\left(e^{+} e^{-} \rightarrow \text { hadrons }\right)}{\sigma\left(e^{+} e^{-} \rightarrow \mu^{+} \mu^{-}\right)}
$$

one can show that to a first order $R=\Sigma_{i} e_{i}^{2}$, that is to the sum of the squared charges of the quarks which contribute to the process. The presence of an additional light quark would increase the value of $R$ over what has been measured. With this technique a limit $\mathrm{M}_{\text {top }}>30.4$ $\mathrm{GeV} / \mathrm{c}^{2}[21]$ has been obtained at the Tristan $e^{+} e^{-}$collider. 
- Width of the W

CDF[22], UA1[23] and UA2[24] have measured the width of the $\mathrm{W}$ boson $\left(\Gamma_{W}\right)$. If a top of sufficiently small mass existed, then the decay $W \rightarrow t \bar{b}$ would increase $\Gamma_{W}$. By combining the measurements of UA1, UA2 and CDF a limit $\mathrm{M}_{\text {top }}>55 \mathrm{GeV} / \mathrm{c}^{2}$ has been obtained. This limit is independent of the top decay mode.

- Combined fits.

Many parameters within the SM depend on $M_{\text {top }}$ through radiative corrections. During the last data taking period, up to the end of 1992 , the LEP experiments collected about $5 \times 10^{6}$ $Z^{0}$. The precision measurements done with these data have been used to confirm the validity of the Standard Model and to determine the values of its fundamental parameters. The accuracy of these measurements makes them sensitive to $M_{t o p}$ (and to a smaller extent to $M_{\text {Higgs }}$ ) through radiative corrections. The dependence of these corrections on the top mass is quadratic and can provide an indirect determination of $M_{\text {top }}$. The dependence on the Higgs mass is logarithmic, and the available data do not provide a significative constraint on $M_{\text {Higgs. }}$ A fit which combines all LEP measurements yields $\mathrm{M}_{\text {top }}=166_{-19-22}^{+17+19} \mathrm{GeV} / \mathrm{c}^{2}[25]$. If one also takes into account the measurements by UA2 [26]: $\mathrm{M}_{W} / \mathrm{M}_{Z}=0.8813 \pm 0.0041$, by $\mathrm{CDF}[27]: \mathrm{M}_{W}=79.91 \pm 0.39 \mathrm{GeV} / \mathrm{c}^{2}$ and by the neutrino experiments CDHS[28], CHARM[29] and CCFR[30]: $1-\mathrm{M}_{W}^{2} / \mathrm{M}_{Z}^{2}=0.2256 \pm 0.0047$, one finds a value of $\mathrm{M}_{\text {top }}=164_{-17-21}^{+16+18} \mathrm{GeV} / \mathrm{c}^{2}$. Because the measurements are not very sensitive to $M_{\text {Higgs }}$, the fit has been repeated for $M_{H_{i g g s}}=60,300$ e $1000 \mathrm{GeV} / \mathrm{c}^{2}$ and the differences of the resulting values for $\mathbf{M}_{\text {top }}$ has been used to determine the above-quoted systematic uncertainty.

\subsubsection{Direct top searches}

The search for the top quark started in 1977, immediately after the discovery of the $b$ quark at the Tevatron. The apparent geometrical progression of the quark masses $\left(M_{s}=0.5 \mathrm{GeV} / \mathrm{c}^{2}\right.$, $M_{c}=1.5 \mathrm{GeV} / \mathrm{c}^{2}, \mathrm{M}_{b}=5 \mathrm{GeV} / \mathrm{c}^{2}$ ), led initially to an expectation for $\mathrm{M}_{\text {top }}$ around $15 \mathrm{GeV} / \mathrm{c}^{2}$. However, the measurement of $R$ in $e^{+} e^{-}$experiments failed to find evidence for top in this mass region. Many experiments have increased the lower limit on $\mathbf{M}_{\text {top }}$ since then. The 
$\mathrm{UA1}[31]$ and UA2[32] collaborations have set limits at 61 e $69 \mathrm{GeV} / \mathrm{c}^{2}$, respectively. These limits were obtained assuming that top decays via weak charged current, as predicted by the Standard Model. The study of the process $Z^{0} \rightarrow t \bar{t}$ allowed the LEP experiments to reach a top limit of $\mathrm{M}_{\text {top }}>46 \mathrm{GeV} / \mathrm{c}^{2}[34]$, independent of the top decay mode.

Presently (January 1994) the highest limit on the top mass comes from CDF[35] which sets a limit of $M_{\text {top }}>113 \mathrm{GeV} / \mathrm{c}^{2}$ from the $92-93$ data. Within the frame of the $\mathrm{SM}$ one must therefore assume that top decays into a real $\mathrm{W}: t \rightarrow W b$.

\subsubsection{Production and decay of the top quark}

In perturbative QCD, pairs of heavy quark and antiquark are produced directly in partonparton collisions $[36,37]$. At the lowest order in the strong coupling constant $\alpha_{s}$ there are two sub-processes of interest[38]:

1.) Annihilation $q+\bar{q} \rightarrow Q+\bar{Q}$;

2.) Gluon-gluon fusion $g+g \rightarrow Q+\bar{Q}$;

Figure 1.2 shows the first order Feynman diagrams for top production in these sub-processes. For $\mathbf{M}_{\text {top }} \sim 100 \mathrm{GeV} / \mathrm{c}^{2}$ they contribute approximately equally. If instead $\mathbf{M}_{\text {top }} \gg 100 \mathrm{GeV} / \mathrm{c}^{2}$.

s-channel
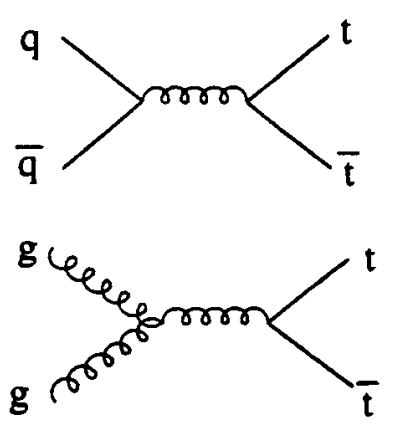

u-channel

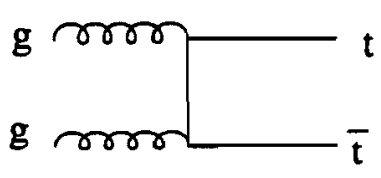

t-channel

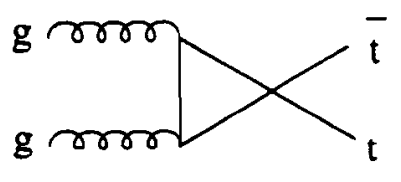

Figure 1.2: Lowest order Feynman diagrams for top production in $p \bar{p}$ collisions. 
process 1.) dominates, since quarks have a harder momentum distribution in the proton compared to gluons. Including higher order corrections introduces new additional production mechanisms. At the $\alpha_{s}^{3}$ order there are the following parton-parton processes :

3.) $q+\bar{q} \rightarrow Q+\bar{Q}+g$;

4.) $g+g \rightarrow Q+\bar{Q}+g$

5.) $g+q(\bar{q}) \rightarrow Q+\bar{Q}+q(\bar{q})$,

together with the virtual corrections to the $2 \rightarrow 2$ processes described by 1.) and 2.). The theoretical study[39] of these processes showed that radiative corrections contribute significantly to the cross section for $t \bar{t}$ production, especially through process 4.). One finds a significant $(\mathrm{O}(30 \%))$ increase in the cross section with respect to the predictions at lowest order. This increase is mostly due to the emission of low energy, collinear gluons in the initial state. These corrections become important especially near "threshold", that is when the energy in the center of mass system $(\sqrt{s})$ is equal to $2 \cdot \mathrm{M}_{\text {top }}$. On the basis of the above-mentioned mass limits, one observes that the available sub-process energy at the Tevatron is not much larger than the threshold energy. The role of these contributions should therefore be important. In order to get a conservative limit on the top mass one would refer to the lowest theoretically predicted value for the production cross section. If one finds a signal, one would use the best available prediction for the central value of the cross section in order to give an estimate of the mass. It is therefore important to evaluate the systematic errors of the theory. They are obtained from a comparison of the predictions with different values of the renormalization scale as expressed by the $\Lambda_{Q C D}$ parameter. Recently[37] the production cross section has been calculated taking into account the emission of soft and collinear gluons from the initial quarks and gluons. These contributions have been calculated to all $\alpha_{s}$ orders. The uncertainty on $\sigma_{t \bar{t}}$ has been estimated to be about $30 \%$. Figure 1.3 shows these new values of the cross section. Within the SM top decays practically always as:

$$
t \rightarrow b W
$$




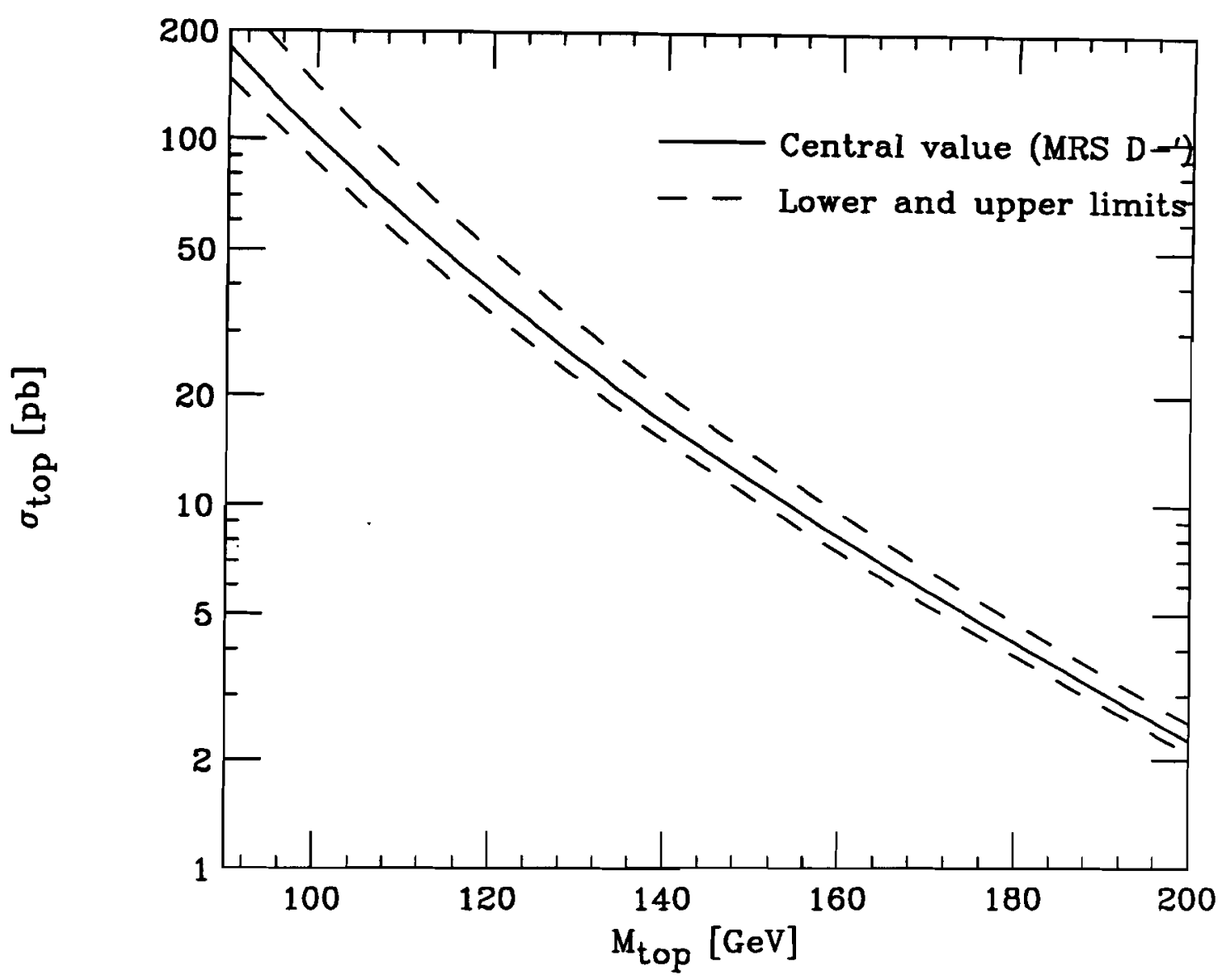

Figure 1.3: Production cross section for $t \bar{t}$ pairs as a function of $M_{\text {top }}$.

Other charged current decays into $W d$ or $W s$ are suppressed because the corresponding elements of the Kobayashi-Maskawa matrix are small[2]. Neutral current decays into $u Z$ or $c Z$ are suppressed by the GIM mechanism. The SM predicts that if $M_{\text {top }} \sim M_{W}+M_{b}$ the top lifetime $\tau=1 / \Gamma$ would be long enough to allow for the fragmentation process to take place, so that a top hadron be formed before the top decays. On the other hand, if $M_{\text {top }} \gg M_{W}$, then top would decay as a free quark. In this regime its width becomes

$$
\Gamma(t \rightarrow W b) \sim 0.17\left(\frac{M_{\text {top }}}{M_{W}}\right)^{3} G e V
$$

The $\mathrm{W}$ from top decay can decay into three different kinds of leptons and two different quark families with three different colors, which makes a total of 9 possible final states. These states occur with the same probability (if we ignore the small phase space effects), and the branching ratio for each of these modes is about $\frac{1}{9}$ (see table 1.4). Figure 1.4 illustrates the production 


\begin{tabular}{|c|c|c|}
\hline \multicolumn{2}{|c|}{ Decay Mode } & Decay Branching Ratio \\
\hline hadronic & $t \bar{t} \longrightarrow q \bar{q} b q \bar{q} \bar{b}$ & $36 / 81(44.5 \%)$ \\
lepton + jets & $t \bar{t} \longrightarrow q \bar{q} b l \nu \bar{b}$ & $36 / 81(44.5 \%)$ \\
dilepton & $t \bar{t} \longrightarrow l \nu b l \nu \bar{b}$ & $9 / 81(11 \%)$ \\
\hline
\end{tabular}

Table 1.3: Decay modes for a $t \bar{t}$ pair and their branching ratio assuming charged currents for the decay. The symbol $q$ means a light quark : u,d,c,s. The symbol l means a charged lepton: $e, \mu$ or $\tau$.

and decay of a $t \bar{t}$ pair. Top events are therefore of the type :

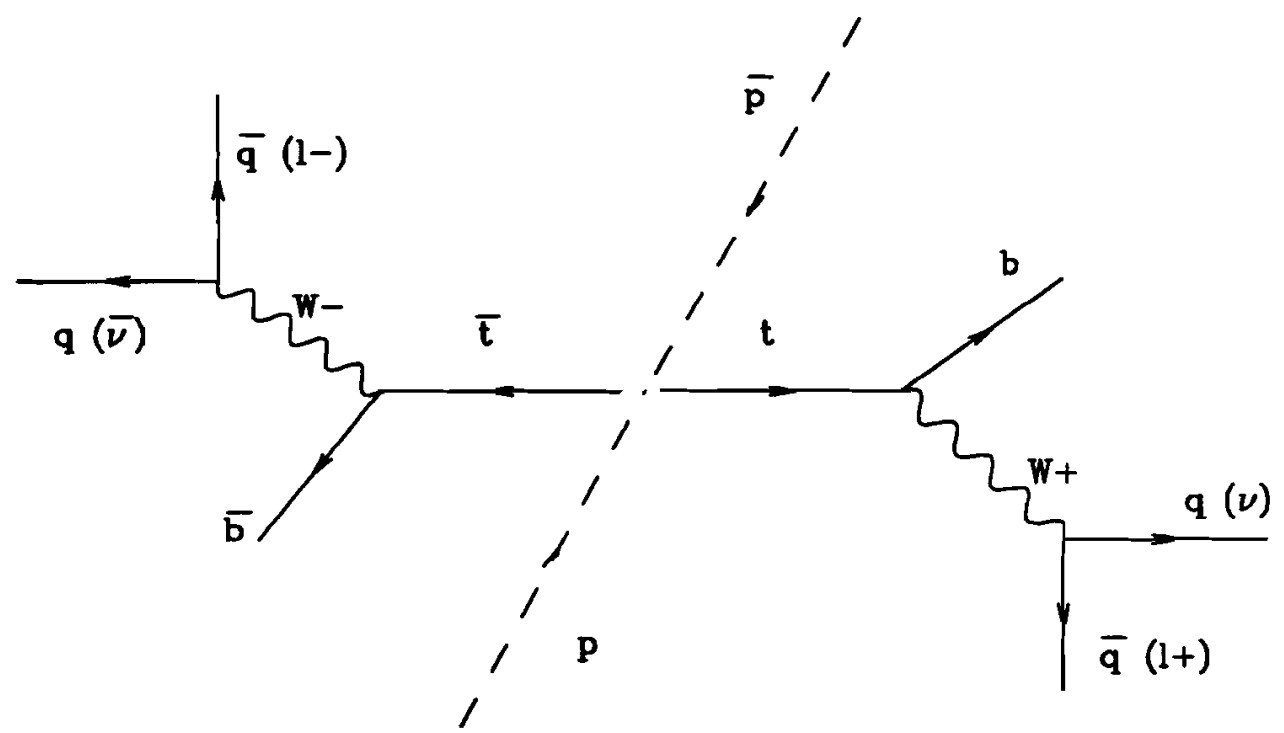

Figure 1.4: Top production in $q \bar{q}$ annihilation followed by the decay chain predicted by the Standard Model.

$$
p \bar{p} \rightarrow t \bar{t} \rightarrow\left(b W^{+}\right)\left(\bar{b} W^{-}\right)
$$

where the quarks appear as jets, and each $W$ boson (real for $M_{\text {top }}>M_{W}$ ) leads to either an energetic charged lepton and a neutrino, or to a pair of jets.

The top search splits naturally into three channels, determined by the decay modes of the two W bosons. If both W's decay hadronically, the event will contain only jets (hadronic mode). If only one of the W's decays hadronically, the event contains a high $\mathrm{P}_{t}$ charged lepton, a neutrino and jets (lepton + jets mode). If both W's decay leptonically, the event contains two high $\mathrm{P}_{t}$ charged leptons, two neutrinos and two jets (dilepton mode). 
The hadronic mode

A large fraction of the events occurs in the final state with only jets (branching ratio of $44 \%$ ). However, this mode suffers from a large QCD background, and estimates show that one may hope to exploit it only if at least one of the $b$ jets can be identified. If this is possible, one might achieve a signal/background ratio of about one third. An attempt to study the purely hadronic channel was done by CDF based on the 1992-93 data[40].

\section{The dilepton mode}

This channel has the smallest branching ratio, but it has the advantage of a relatively small background. If the top mass is very large, the background from WW production can become important. One possibility to solve this problem is to require two jets (from the decays of the $b$ quarks). The $b$ jets are expected to be of increasingly high $\mathrm{P}_{t}$ for increasing top masses. Additional signatures can come from the identification of $b$ quarks in the jets, either by identifying $b$-decay vertices, or semileptonic decays of the $b$.

\section{The lepton + jets channel}

In this channel, the charged leptons which allow a relatively easy detection are the electron and the muon. As a consequence, the usefull rate lies between those of the hadronic and of the dilepton channel, with a branching ratio of about $15 \%$ for each type of charged lepton. The physical background comes from pair production of lighter quarks, mostly $b$ quarks, and from QCD production of W's with associated jets. It is relatively easy to eliminate the $b \bar{b}$ background, for example by requiring the presence of energetic neutrinos ( $F_{t}$ or missing transverse energy), since the mass difference of $b$ and top quarks is large. The remaining background is then from QCD W+jets production[41]. When the number of jets is equal to the one predicted for the process $t \bar{t}$, these events are 2 to 10 times more frequent then $t \bar{t}$ events, depending on the top mass and on the cuts which are imposed on $E_{t}$ (jets). A detailed study of the kinematics of these events is necessary in order to identify the signal. The most recent top limit from $\mathrm{CDF}\left(\mathrm{M}_{\text {top }}>113 \mathrm{GeV} / \mathrm{c}^{2}\right.$ at $95 \%$ C.L.[35]) comes from the 
(small) number of observed di-lepton events. Also the lepton + jets channel has been used in the past, yielding a limit of $\mathrm{M}_{\text {top }}>77 \mathrm{GeV} / \mathrm{c}^{2}[42]$. That study was based on a comparison of the measured distribution $\mathrm{M}_{\text {trans }}$ of the system $e+W_{t}$ with the one expected for a mixture of top events and QCD W events. This kind of analysis had also been previously done by $\mathrm{UA2}$ [32] and gave a limit of $\mathrm{M}_{\text {top }}>69 \mathrm{GeV} / \mathrm{c}^{2}$. If $\mathrm{M}_{\text {top }}<\mathrm{M}_{W}$, it will decay into a virtual $\mathrm{W}$. The transverse mass distribution of the virtual W's will be different from that coming from a real W. This analysis is not applicable when $M_{t o p} \geq M_{W}+M_{b}$, because in this case also the top-decay $\mathrm{W}$ is real. For this reason the single lepton channel in the past has produced less restrictive limits than the dilepton channel, in spite of the larger number of expected events. This channel becomes again important for the search of a very heavy top. One can make use of the event structure which is different for QCD W and top events[33]. In addition, also in this channel, as for the dilepton channel, the identification of $b$ quarks in the jets can be of great help.

This approach is the subject of this thesis. In the rest of this chapter we present the basic idea, and describe a first feasibility study.

\subsection{Exploiting the Single Lepton Event Structure}

We expect that the structure of $\mathrm{W}+$ jet events from QCD and from $t \bar{t}$ decays will be different because the matrix elements of the two processes are different.

\subsubsection{W+jets from QCD}

We begin with a more detailed look at the process which generates the main background for the top search in the single lepton channel: QCD production of W's with jets. In parton collisions the $\mathrm{W}$ can be produced together with one or more energetic quarks or gluons. Some of the contributing diagrams are shown in figure 1.5. The diagrams 1.5 (b) show the $\mathrm{W}$ recoiling against a quark or gluon. The diagrams of higher order can have a second parton in the final state, which can generate more than one jet in the event. The matrix element of these processes is proportional to a power of the coupling constant $\alpha_{s}\left(\mathrm{Q}^{2}\right)$. This coupling constant 


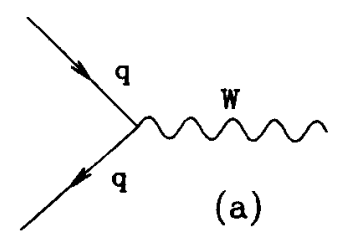

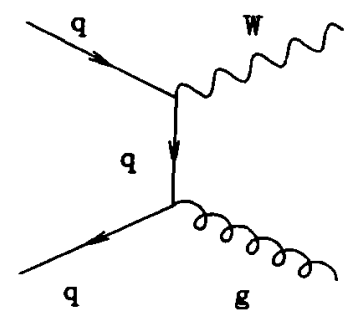

(b)

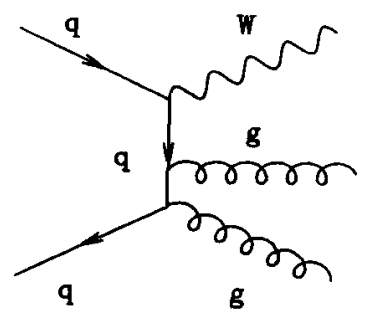

(d)

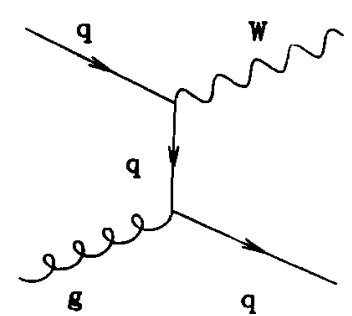

(c)

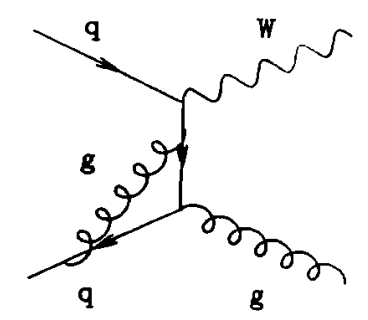

(e)

Figure 1.5: Some of the Feynman diagrams for $W$ production. The process at lowest order is shown in (a). Some examples for higher order processes are shown in (b), (c), (d), (e).

depends, as we have discussed, on the $Q^{2}$ scale at which the parton collision happens. Since one is dealing here with a large momentum transfer, one can get a fair approximation by using perturbative QCD. A good reference point for the constant $\alpha_{s}$ is the mean value of the LEP measurements, i.e. $\alpha_{s}\left(M_{Z}{ }^{2}\right)=0.1134 \pm 0.0035$.

From what has been previously discussed it is clear that a reliable calculation for the production of multi-jet final states is very important for the top search. Unfortunately the calculation of these processes, which are the physics background for the top search, is complicated. Recently new mathematical methods have been developed which make it possible to simplify the calculation of the matrix elements in lowest order ('tree level') for processes with $n$ partons, allowing to perform the calcultion up to $n=6[43]$. In these calculations it is also necessary to apply cuts on the phase space of the outgoing partons. To understand why, one can consider 
the simplest process $g g \rightarrow g g$. The scattering of the four gluons at tree level (i.e. without taking into account loops) is a lowest order process $\left(O\left(\alpha_{s}^{2}\right)\right)$. To calculate the next order in $\alpha$ one needs to take into account contributions from the scattering of the four gluons (but now with loop corrections) and the scattering of 5 gluons at tree level. The contributions from loops to the four gluons scattering contain divergencies. The ultraviolet divergencies can be absorbed in the renormalization of the coupling constant. However, there are also singularities in the five-gluon matrix element. They occur when two gluons are collinear, or when the energy of one of them comes close to zero. In a more experimentally oriented language this means that two of the outgoing gluons will create a single jet, or that the jet is too close to the beam, or it is not energetic enough to be observed. As a consequence not all of the 5 gluons scattering processes will be observed as events with 3 jets. Some will rather generate events with 2 jets in the final state. Therefore, if one wants to determine the cross section for the two jets final state, one needs to add the 5 gluons process to the loop corrections of the 4 gluons processes. The infrared singularities cancel, while the divergencies due to the initial state collinearities remain. These divergencies are reabsorbed in the initial state parton structure functions. It follows therefore, that the resulting cross section will depend on how jets are defined, for example on what angle of separation is required to identify two partons as two different jets. These jet definitions need to be considered when choosing the cuts in phase space so that it is adequate to the experimental situation. Let's suppose, for example, that we calculate the 3 jets final state and require some minimum separation between the outgoing partons and a minimum energy for each of these partons. There are events which will not pass these cuts and will therefore contribute to the missing higher order corrections for the two jets case. The events which pass the cuts will be instead part of the lowest order contributions to three jets production. One finds that corrections introduced at the tree level influence mostly the absolute value of the cross section, and have little effect on the shape of distributions. By accounting for higher order corrections, one can reduce the dependence on the $Q^{2}$ scale. This scale needs to be chosen, according to the process of interest. Once we want to compare the results of the calculations to the experimental data we have to remember that: 
- The overall normalization is not known

- The results depend on the choice of the $\mathrm{Q}^{2}$ scale.

To quantify the uncertainty in the normalization, one introduces the " $k$ factor". This factor is a constant by which one multiplies the cross section at tree level to obtain the correct absolute value for the cross section. This factor depends on the process, on the $Q^{2}$ scale, on the center of mass energy and possibly on other parameters. For example, for 4-jet production at CERN, the $\mathbf{k}$ factor obtained from comparing the experimental results with tree level calculations was $\mathrm{k}=1.35 \pm 0.08[44]$. If one multiplies the theoretical predictions at tree level by this factor, one gets an excellent agreement between theory and experiment for all relevant distributions of the 4 -jet production process.

\subsubsection{Montecarlo simulations}

We need to find out in more detail how useful the kinematical differences between top and QCD are for the top search. As a first step we did a Montecarlo study of the event structure at the parton level[45], without simulating the detector response. Two Montecarlo programs were used: Papageno[46] to simulate $t \bar{t}$ events (without the production of additional jets), and Vecbos[47] for the simulation of the QCD background. Both Montecarlos are based on the matrix element calculated at lowest order, and were extensively used in the past for many different studies. The development of Vecbos, the first Montecarlo which provides the matrix element for $W+j e t$ events with up to four (partons) jets, was possible because of the development of the mathematical techniques previously described. This analysis makes frequent use of it, and will be described in more detail in Chapter 4. We generated $10^{5}$ top events at a mass of $100 \mathrm{GeV} / \mathrm{c}^{2}, 10^{6} \mathrm{~W}+3$ parton events and $5 \cdot 10^{5} \mathrm{~W}+4$ parton events. The following cuts on phase space were applied during generation:

- $\mathrm{P}_{t}($ parton $)>10 \mathrm{GeV} / \mathrm{c}$;

- $\Delta \mathrm{R}($ parton-parton $)>0.6^{1}$

\footnotetext{
${ }^{1} \Delta \mathrm{R}$ is defined as $\sqrt{\Delta \eta^{2}+\Delta \phi^{2}}$, where $\eta$ is the pseudorapidity and $\phi$ the azimuthal angle around the beam axis.
} 
- $\mid \eta($ parton $) \mid<3.5$.

After event generation we required:

- $\mathrm{P}_{t}$ (charged lepton $)>20 \mathrm{GeV} / \mathrm{c}$;

- $\mathrm{M}_{\text {trans }}(e+\nu)>40 \mathrm{GeV} / \mathrm{c}^{2}$;

- $\mathrm{P}_{t}(\nu)>20 \mathrm{GeV} / \mathrm{c}$

- $\mid \eta($ charged lepton $) \mid<1.2$.

\subsubsection{Angular correlation between proton beam and hadronic ac- tivity}

In events $t \bar{t} \rightarrow b \bar{b} l \nu j j$ two of the jets will have the invariant mass of the W. In QCD processes this correlation does not exist. However, it has been claimed[48] that this method by itself is not sufficient to beat the background because of the bad signal/noise ratio and because of experimental uncertainties.

On several occasions it has been shown [49] that in QCD jet production the jets tend to be emitted in the forward direction, close to the beam axis. On the contrary in the decay of a centrally produced heavy object (like $t \bar{t}$ ) the decay products are less correlated with the beam direction. This difference has already been used for various studies, for example in the search for leptoquarks[50]. Those studies showed that a useful variable to express this effect is $\cos \theta^{*}$, where $\theta^{*}$ is the angle between the outgoing particle (in our case a parton) and the incoming proton beam direction in the event rest system.

In this thesis we study W's which decayed leptonically. The longitudinal component of the neutrino momentum cannot be measured at a hadron collider, therefore the system of the $W$ and the three leading jets cannot be fully reconstructed. The jet angular distributions in top events and in pure QCD events were compared in three different systems:

- The true rest system, which is known in Montecarlo events;

- The rest system, not accounting for the neutrino longitudinal momentum; 
- The laboratory system.

One finds that the analysis described here can be done in any of these systems: the differences in the discrimination power between signal and background are small.

\subsubsection{Classification of the event sample}

In top events, the number of jets passing the analysis cuts is not always the same. In this study we discuss $W$ events with three or more partons. The fraction of events which will contain four (or more) jets depends on the top mass. We began with a study of QCD W events with three partons. These events are expected to dominate the inclusive sample of $\mathrm{W}+n$ partons, $n \geq 3$. In a next step we analyzed what happens when we include $W+4$ parton events. At the time being it is not possible to study $W+5$ parton events because there is no Montecarlo with $\mathrm{W}+5$ parton matrix elements.

\subsubsection{Signal - Background Separation}

The partons were ordered according to their values in $\mathrm{P}_{t}$, parton $_{1}$ being the one with the highest $P_{t}$. A cut $P_{t}$ (parton) $>15 \mathrm{GeV}$ was applied: at this value of $\mathrm{P}_{t}$ the experimental jet reconstruction is already reasonably good. Initially, possible additional jets were not considered. The effects from additional partons will be discussed later. Figure 1.6 shows the distribution of $\cos \theta_{m a x}^{*}$, which is the maximum of $\mid \cos \theta^{*}\left(\right.$ parton $\left._{1}\right) \mid$ and $\mid \cos \theta^{*}\left(\right.$ parton $\left._{2}\right) \mid$. The distributions shown are for $Q C D W+3$ parton events and for top. As expected, the partons in $Q C D$ events tend to be emitted into the forward direction, the decay products of top are more central. If one requires $\cos \theta_{\max }^{*}<0.8$, the signal to noise ratio improves by a factor of 1.85 (for the $W+3$ jet sample the statistical error on the quoted numbers is always less then $10 \%$ ). The $\mid \cos \theta^{*}\left(\right.$ parton $\left._{3}\right) \mid$ distribution is shown in figure 1.7 , after applying the cut $\cos \theta_{\text {max }}^{*}<0.8$. Again, the top events are more central. An additional cut at $\mid \cos \theta^{*}\left(\right.$ parton $\left._{3}\right) \mid<0.8$ improves the signal to noise ratio further, by a factor of 1.7. The relative normalization of top and QCD events in figures 1.6 and 1.7 is done in such a way that after applying the cuts in $\cos \theta^{*}$ the number of top and QCD events is the same. Applying these cuts reduces the number of QCD events by 


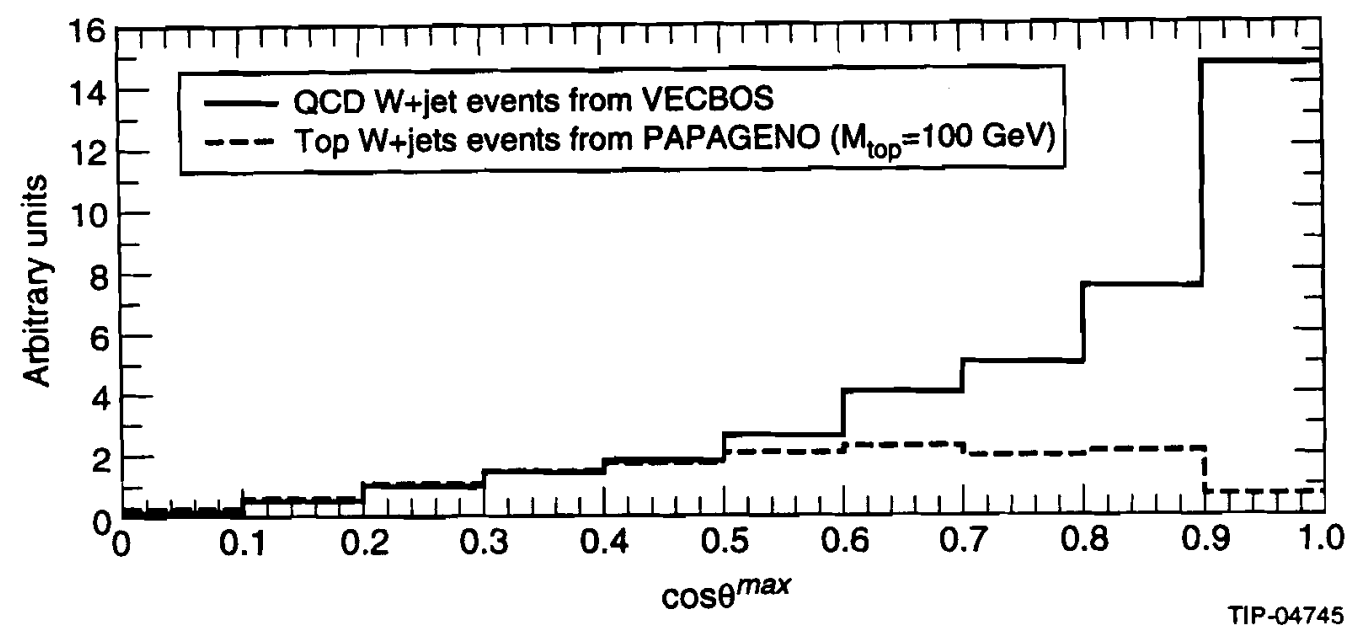

Figure 1.6: Distribution of $\cos \theta_{\max }^{*}$, which is the maximum of $\mid \cos \theta^{*}($ parton 1$) \mid$ and $\mid \cos \theta^{*}\left(\right.$ parton $\left._{2}\right) \mid$

a factor of 4 , while only $30 \%$ of top events is lost. The efficiencies of the cuts are summarized in table 1.4. This study has been done for a top mass of $100 \mathrm{GeV} / \mathrm{c}^{2}$, but the same principle also works for higher top masses.

\begin{tabular}{|l|lc|}
\hline Cut & QCD & top \\
\hline $\cos \theta^{\max }<0.8$ & 2.3 & 1.3 \\
\hline $\cos \theta^{\star}\left(\right.$ jet $\left._{3}\right)<0.8$ & 1.7 & 1.1 \\
\hline \hline Total & 3.9 & 1.4 \\
\hline
\end{tabular}

Table 1.4: Reduction factors of the $\cos \theta^{\star}$ cuts.

\subsubsection{Estimate of the systematic errors}

\section{Experimental jet reconstruction}

In an experiment it can happen that a shower from a parton of $P_{t}$ (parton) $>15 \mathrm{GeV}$ is reconstructed as a jet of $E_{t}(j e t)<15 \mathrm{GeV}$, or viceversa. $E_{t}$ (jet) and $\cos \theta^{*}$ (jet) are correlated in the sense that jets of different $E_{t}$ may have different distributions in $\cos \theta^{*}$. Therefore the effect of the $\cos \theta^{*}$ cut on the signal to noise ratio may be different for experimentally reconstructed jets. This problem has been studied using again the $W+3$ partons sample and asking again 


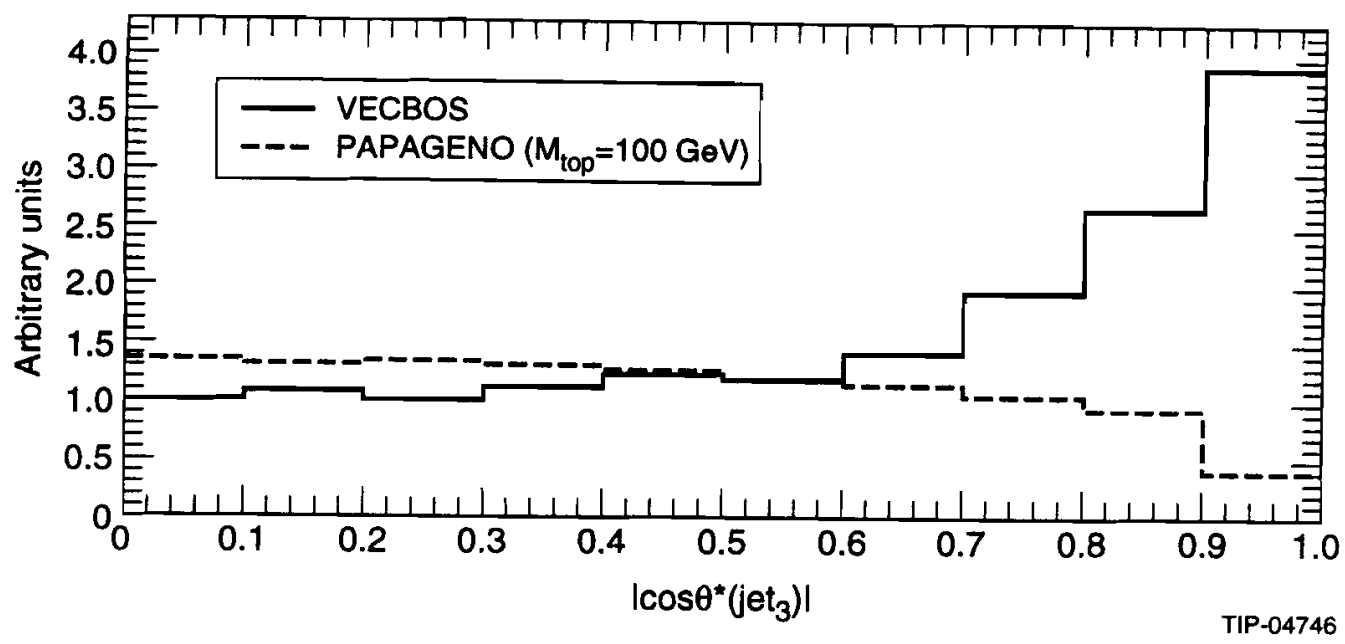

Figure 1.7: Distribution of $\mid \cos \theta^{*}\left(\right.$ partone $\left._{3}\right) \mid$ after applying the cut $\cos \theta_{\max }^{*}<0.8$. Vecbos and top are normalized to each other in the region $\mid \cos \theta^{*}\left(\right.$ parton $\left._{3}\right) \mid<0.8$.

$\mathrm{P}_{t}\left(\right.$ parton $\left._{1,2}\right)>15 \mathrm{GeV}$, but now with the requirement $10 \mathrm{GeV}<\mathrm{P}_{t}\left(\right.$ parton $\left._{3}\right)<15 \mathrm{GeV}$ (instead of $\mathrm{P}_{t}\left(\right.$ (parton $\left._{3}\right)>15 \mathrm{GeV}$ as before). With these cuts on $\mathrm{P}_{t}$ (parton), the $\cos \theta^{*}$ cuts reduce the sample by a factor of 4.5. The background reduction has therefore increased: it was 3.9 before. For top events the spectrum $\mathrm{P}_{t}\left(\right.$ parton $\left._{3}\right)$ does not fall so much towards higher $\mathrm{P}_{t}$. Therefore the energy smearing does not affect the top events.

\section{Jet multiplicity}

One also needs to take into account $W$ events with more than 3 partons for two reasons:

1) As the top mass increases, more and more top events will have a fourth jet. If one does not include $\mathrm{W}+4$ jet events in the analysis, a large fraction of the signal may get lost.

2) In the experimental data an event with 4 jets (partons) may appear as a $W+3$ jet event if one of the jets is not reconstructed.

We limited our investigation of this problem to $\mathrm{W}+4$ jet events, since there is no $\mathrm{W}+5$ partons matrix element Montecarlo presently available. The $\cos \theta^{*}$ cuts, as described before, have been applied to $\mathrm{W}+4$ parton events. A reduction factor of 3.7 has been obtained, with a statistical error of $25 \%$ (the Montecarlo production of $W+4$ jet events requires much computer time and 
so we were not able to produce a sample large enough to ignore statistical errors). Within the statistical uncertainties, one can conclude that $W+4$ jet events get reduced by about as much as $\mathrm{W}+3$ jet events, after applying the $\cos \theta^{*}$ cuts. Therefore it should be possible to enrich a $\mathrm{W}+3$ (or more) jets sample with top events, making use of the differences in the event structure. One could imagine looking for top in the experimental data simply by comparing the number of events with central jets to the number of events with forward jets. However, one would obtain uncertainties on the expected number of events which presumably would be too large to arrive at a conclusive result.

A more promising strategy would be to first apply the cuts in $\cos \theta^{*}$ in order to suppress the background. In the remaining events one could study other variables which distinguish between signal and background. Examples are $M_{j j}, E_{t}\left(\right.$ jet $\left._{3}\right)$ or $E_{t}\left(\right.$ jet $\left._{4}\right)$. It should be possible to obtain information on the top quark in this way. The limitation of this method will be given by the systematic errors of the available Montecarlo simulations. A further improvement of these already sophisticated programs will require much effort and time. However, significant progress could come in the meantime from a comparison between existing calculations and experimental data. To this purpose, the analysis described in the following was applied to the CDF data. Such an analysis was initially designed to search for a top of $100 \mathrm{GeV} / \mathrm{c}^{2}$ or more. The results obtained with the 88-89 data[51] showed some events which are difficult to explain with QCD, and suggested that top quark must be heavy (see Appendix B). For this reason in the 92-93 data analysis, the subject of this thesis, we restricted ourselves to the search of a top with $\mathrm{M}_{\text {top }}>140 \mathrm{GeV} / \mathrm{c}^{2}$. 


\section{Chapter 2}

\section{The Experimental Apparatus}

In this chapter the hadronic collider Tevatron at Fermilab is described. This thesis uses data taken by the CDF detector in 1992-1993. Also the detector is briefly described, giving prominence to those parts which were relevant for the analysis.

\subsection{The Tevatron Collider}

The Tevatron collider at Fermilab (USA), is an accelerator ring which uses superconducting magnets. It produces $p \bar{p}$ interactions at a center of mass energy $(\sqrt{s})$ of $1.8 \mathrm{TeV}$, presently the greatest available in the world. The energy which can be provided by an accelerator is an important parameter for the study of several physics processes such as high momentumtransfer $\left(\mathrm{Q}^{2}\right) \mathrm{QCD}$ scattering, $\mathrm{W}$ production, and the production of heavy quarks. This is because these processes require a very energetic partonic collision in order to produce high masses and $\mathrm{Q}^{2}$. The partons in the proton have an $x=P_{t}($ parton $) / P_{t}$ (proton) distribution which is peaked at low $x$ value $(x \sim 0.15)$. For this reason to have an high flux of energetic partons, one should start from very energetic protons. At the Tevatron (showed in figure 2.1), beams of proton $(p)$ and anti-proton $(\bar{p})$ collide after the following steps:

- Production of the proton bunches.

The process starts with the ionization of gaseous hydrogen to have $\mathrm{H}^{-}$ions. These ions are then accelerated first with a Cockroft-Walton accelerator until they reach an energy of 750 $\mathrm{KeV}$, and then with a linear accelerator (Linac) wich brings them to $200 \mathrm{MeV}$. After they are 


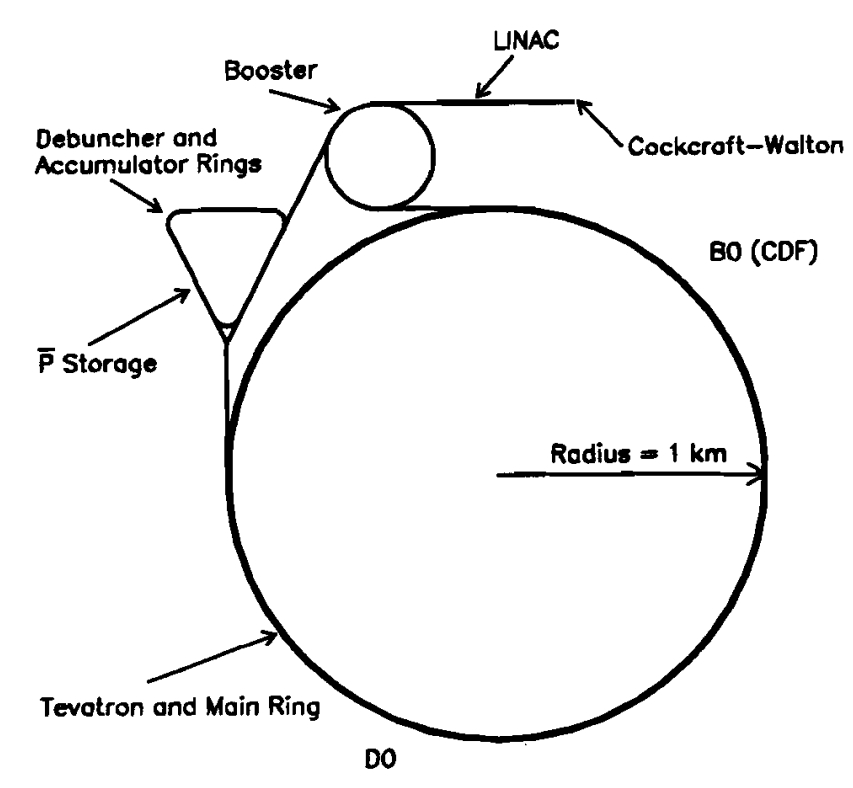

Figure 2.1: Schematic view of the Tevatron.

focused, they are sent against a thin carbon foil. Due to this interaction they lose 2 electrons, becoming protons. These protons are transferred to a circular accelerator (the Booster, a synchrotron with $75 \mathrm{~m}$ radius) and brought to an energy of $8 \mathrm{GeV}$. At this point, using the accelerating radio-frequency, the protons are grouped in bunches of about $10^{10}$ particles. These bunches are then injected in the Main Ring, a synchrotron of the same dimensions of the Tevatron $(\mathrm{R}=1 \mathrm{Km})$ and located in the same tunnel. Conventional magnets drive the bunches until they reach an energy of $150 \mathrm{GeV}$. Soon afterwards, the protons are transferred into Tevatron.

- Production of antiproton bunches.

A fraction of the protons in the Main Ring, once they have reached the energy of $120 \mathrm{GeV}$, are extracted from the ring and sent against a target to produce antiprotons. The goal is to produce and accumulate a large number of antiprotons, reducing their momentum spread and their angular divergency. In this way they can be transferred with high efficiency into the Main Ring and afterwards into the Tevatron, in dense bunches of small cross sections. This is necessary in order to obtain a high luminosity from the collider. To this purpose, the antiproton beam is focalized through a parabolic magnetic lithium lens. It is then transferred to another 
accelerator ring called the Debuncher. Inside the Debuncher, a suitable rotation in the phase space disperses the temporal structure, improving the monocromaticity of the longitudinal momentum. The antiprotons are then transferred to the accumulation ring (Accumulator) and stored there for thousands of pulses. A stochastic cooling system reduces the momentum spread in all three directions. The stochastic cooling system is made of several "pickups", amplifiers and "kickers". The pickups detect locally the deviation of the antiproton bunches from the main orbit in the Accumulator. The signal coming from the pickups is then amplified and sent to kickers located at opposite azimuthal angles along the ring. The kickers produce an electromagnetic field, which corrects the deviation detected by the pickups. When about $60 \times 10^{10}$ antiprotons are accumulated, six bunches of about $4 \times 10^{10}$ antiprotons are transferred in the Main Ring, and then sent to reach the protons in the Tevatron at $150 \mathrm{GeV}$.

- $\underline{p \bar{p}}$ interactions.

At this moment, six bunches of protons and six of antiprotons travel inside the Tevatron in opposite directions. They are then accellerated to an energy of $900 \mathrm{GeV}$, and they collide in the two interaction regions $(B 0$ e $D 0)$ where the CDF and D0 detectors are located. The time interval between two collisions is $3.5 \mu \mathrm{s}$.

In order to detect processes characterized by a small production cross section, as for example the top quark production, a large number of $p \bar{p}$ collisions is necessary. The number $\mathrm{N}$ of collisions is given by:

$$
N=L \sigma
$$

where $\sigma$ is the production cross section for that process and $L$ is the luminosity (whose dimensions are therefore in $\mathrm{cm}^{-2} \mathrm{sec}^{-1}$ ). For particle bunches characterized by a revolution frequency of $f_{0}$ (in the case of the Tevatron: $\mathrm{f}_{0}=50 \mathrm{KHz}$ ) the luminosity is defined as:

$$
L=\frac{N_{p} N_{\bar{p}} B f_{0}}{4 \pi \Sigma}
$$

where $N_{p}\left(N_{\bar{p}}\right)$ is the number of protons (antiprotons) in each bunch, $B$ is the number of bunches of prontos (antiprotons), and $\Sigma$ is the transverse cross section of the bunches. $\Sigma$ is determined by the beam parameters and by the magnetic focusing properties of the accelerator. 
To increase the luminosity at the two interaction regions, a complex system of magnetic lenses is used. The high focusing provided by these lenses reduces as much as possible the beam cross section at the crossing point.

During the 1992-93 run, from May the 12th 1992 to June the 1st 1993, the average luminosity was $3 \times 10^{30} \mathrm{~cm}^{-2} \mathrm{sec}^{-1}$ and a maximum luminosity of about $9 \times 10^{30} \mathrm{~cm}^{-2} \mathrm{sec}^{-1}$ was reached. The integrated luminosity collected by CDF was about $22 \mathrm{pb}^{-1}$, with an efficiency near $75 \%$.

\subsection{The CDF Detector}

The Collider Detector at Fermilab (CDF[52, 53]) was built to measure the energy and momentum of electrons, muons, hadronic jets and neutrinos produced in the Tevatron collisions, covering the widest possible solid angle. A schematic view of CDF is shown in figure 2.2. For this thesis we used primarily the central part of the detector, which therefore will be described in more detail. For the following discussion one needs to introduce the coordinate system used by CDF, given by two sets of cartesian and polar coordinates respectively.

Cartesian coordinates:

1. $z$ axis: defined by the beam direction ( $z>0$ is the proton direction);

2. $x, y$ plane: orthogonal to $z=0$ it is the CDF median plane and it contains the nominal center of interactions. The $\boldsymbol{x}$ axis is conventionally taken on the horizontal plane, oriented along the radial direction coming out from the accelerator.

Polar coordinates:

1. $\phi$ : azimuthal angle in the counter-clockwise direction around the $z$ axis, starting from the $x$ axis;

2. $\theta$ : polar angle relative to the oriented $z$ axis;

3. $r$ : radial distance from the $z$ axis. 


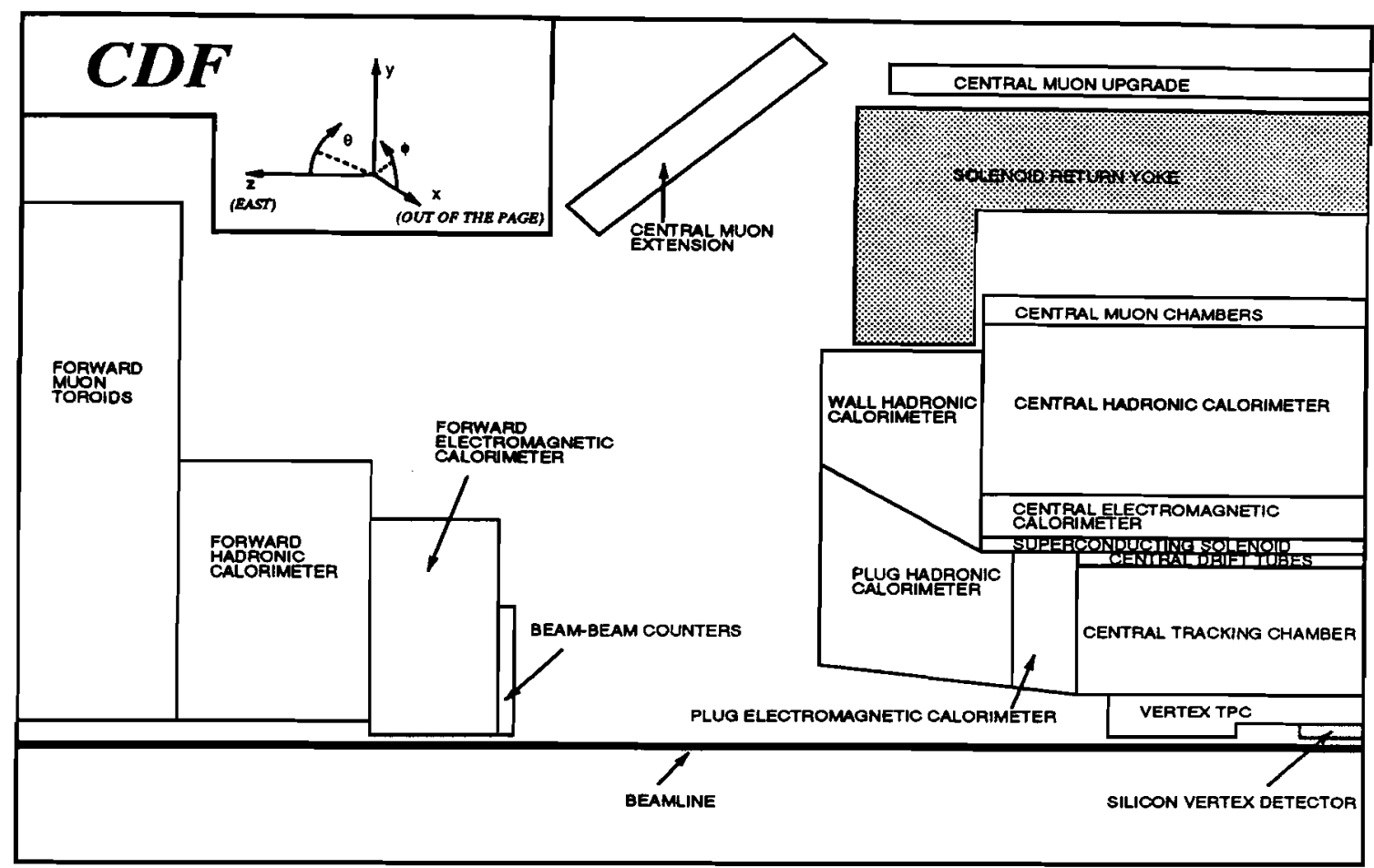

Figure 2.2: Side-view cross section of the CDF detector. The detector is forward-backward symmetric about the interaction region. In the upper-left corner of the figure the coordinate system is also shown.

The CDF detector is azimuthally as well as backward-forward symmetric about the interaction region. Starting from the nominal point of interactions, three main components are distinguishable: the tracking system, the calorimeters, and the muon detectors.

\subsubsection{The tracking system}

The whole tracking system resides in an axial magnetic field of 1.4 Tesla, generated by a superconducting solenoid with its axis parallel to the beams. The magnet has a length of 4.8 $\mathrm{m}$ and a radius of $1.5 \mathrm{~m}$. The tracking system is subdivided as follows: 


\section{SVX}

The first element, located immediately outside the vacuum pipe where the beams are circulating, is a silicon vertex detector (SVX). The SVX was installed in CDF in 1992 and consists of 4 layers of semiconducting silicon detectors. The electrodes are made of microstrips. The SVX [54] is used to identify secondary vertices from $b$ quark decays. To this purpose it provides high resolution tracking in the $r-\phi$ plane, using tracks previously reconstructed in the CTC. The SVX is $51 \mathrm{~cm}$ long, and is divided in two identical cylindrical modules ("barrel") which meet at $z=0$. Since $p \bar{p}$ interactions are spread according to a gaussian distribution with $\sigma=30$ $\mathrm{cm}$ around the interaction point along the $z$ axis, the SVX acceptance is not complete, but it is about $60 \%$. Each barrel is organized in 12 azimuthal sections ("wedges"). Finally each section contains a radial telescope of 4 detector layers ("ladders"). The electrodes are parallel to the beam direction. The four layers are between 3 . and $7.9 \mathrm{~cm}$ from the beamline. The individual SVX hit resolution is measured in data to be $\sigma=13 \mu \mathrm{m}$. If the SVX information is used to determine track momenta, the momentum resolution improves by a factor 2 , up to $\frac{\delta P_{t}}{P_{t}}=0.001 \times \mathrm{P}_{t}$.

\section{VTX}

Radially after the SVX there is a telescope of octagonal vertex time-projection chambers (VTX)[53]. The VTX was also installed in 1992 and it is able to detect tracks up to a radius of $22 \mathrm{~cm}$ from the beam with $|\eta|<3.25$. The VTX is used to measure the $p \bar{p}$ interaction vertex along the $z$ axis with a resolution of $1 \mathrm{~mm}$, and to distinguish if there were two superimposed interactions.

\section{CTC}

Both the SVX and VTX are mounted inside the central tracking chamber (CTC). The CTC[55] is a large cylindrical drift chamber, $3.2 \mathrm{~m}$ long and with an external radius of $1.3 \mathrm{~m}$. The CTC reconstructs high $P_{t}$ tracks and transfers this information to the first level trigger (see subsection 2.2.4). The CTC also measures the charged track parameters to be used in data 


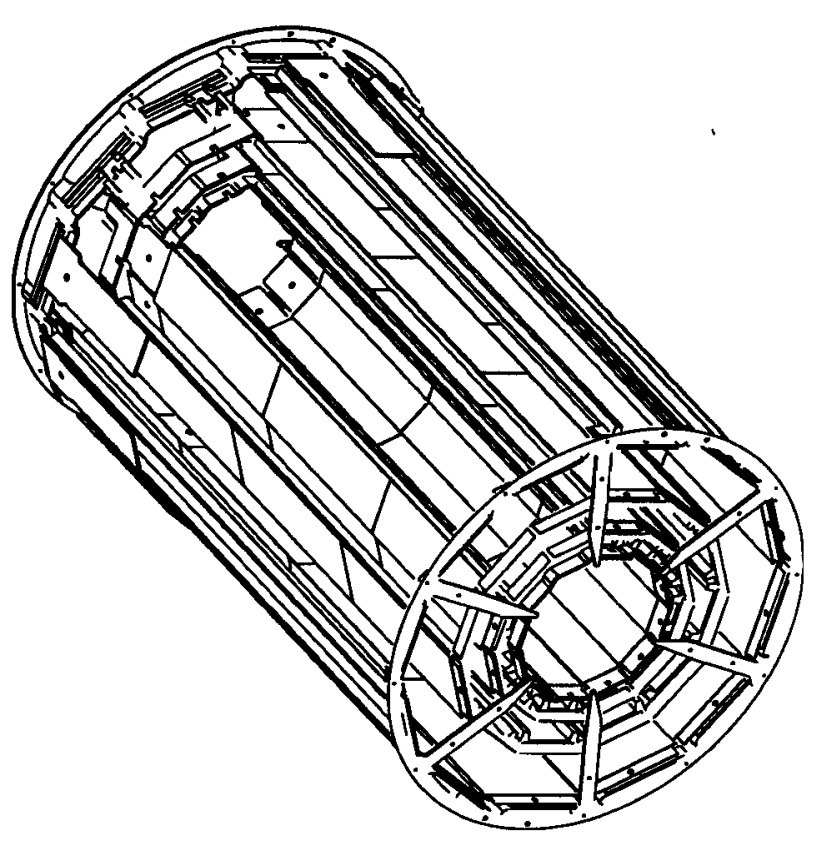

Figure 2.3: Perspective view of one of the two SVX barrels..

analysis. The CTC covers with high efficiency the region $|\eta|<1$ and consists of 84 layers of sense wires organized in 9 cylindrical super-layers. In 5 of these super-layers the wires are parallel to the beam, and provide accurate information ( $200 \mu$ resolution on the individual hit) on the track position in the transverse plane. The remainig four super-layers contain instead wires which are tilted at an angle of $\pm 3^{\circ}$. This stereo effect allows tracks reconstruction also in the $r-z$ plane. The resolution on this measurement is about $4 \mathrm{~mm}$. The information redundancy provided by the CTC allows also the reconstruction of those events with a large number of near tracks and characterized by high $\mathrm{P}_{t}$ jets, as the ones which have been studied in this thesis. The momentum resolution for isolated tracks provided by the CTC is: $\frac{\delta P_{t}}{P_{t}}=0.002 \times \mathrm{P}_{t}$. During the last run, the 54 outer layers of the chamber have been improved. It is now possible to measure the ionization energy $(d E / d x)$ released by the particles, with a resolution of $\sim 15 \%$. This additional information allows more efficient discrimination of electrons from charged 


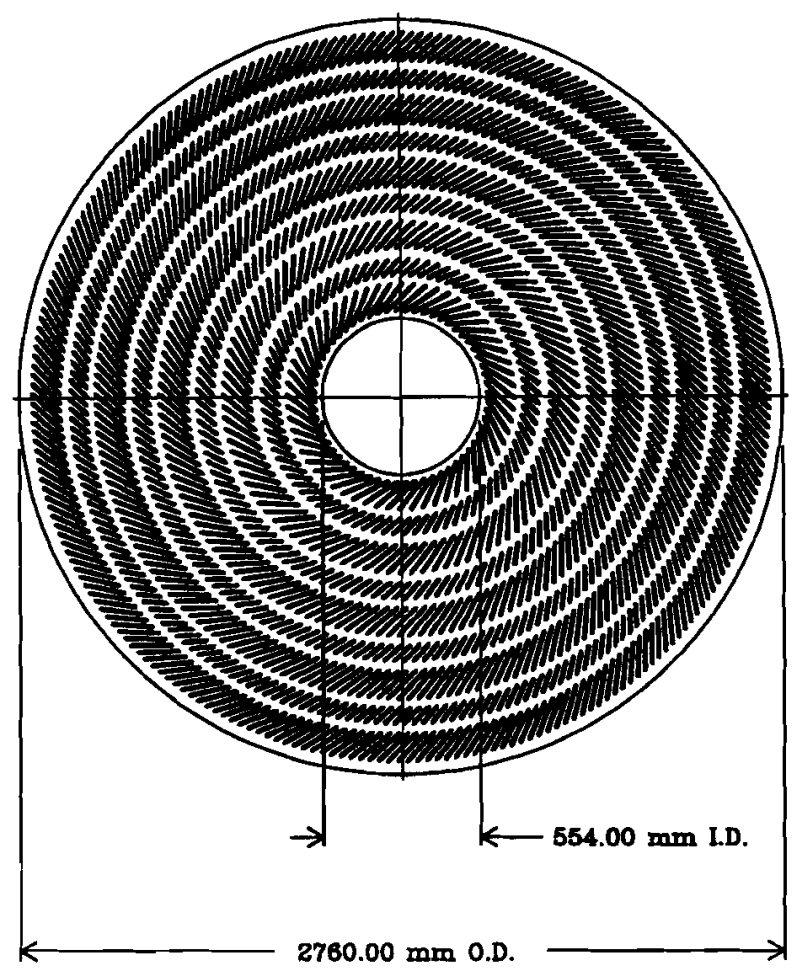

Figure 2.4: Layout of the CTC sense wires. The 9 layers structure is visible. The wires are organized in cells, rotated by about $45^{\circ}$ to take into account the Lorentz angle of the particles which move inside a magnetic field.

pions up to $P_{t}<4 \mathrm{GeV} / \mathrm{c}$ and to separate $K^{ \pm}$from $\tau$ up to $P_{t} \leq 700 \mathrm{MeV} / \mathrm{c}$.

\section{CDT}

Outside the CTC, immediately before the inner surface of the magnetic coil, there are three layers of central drif tubes (CDT)[56]. Each tube, located at a fixed $\phi$, provides accurate information on the track position in the $r-z$ plane.

\subsubsection{Calorimeters}

Outside the magnet there are the central and forward calorimeter systems. Each of the systems is subdivided into an inner electromagnetic part and an outer hadronic part. 


\section{Central calorimetry}

The central electromagnetic (CEM) and hadronic (CHA) calorimeters cover the $|\eta|<1.1$ region, $\left(30^{\circ} \leq \theta \leq 150^{\circ}\right)$. They are sampling calorimeters which use scintillator as the active medium, interleaved with layers of absorber: lead in the CEM and iron in the CHA. An important feature of the CEM and CHA is their segmentation in projective towers. The $z=0$ plane divides the CEM and CHA in two symmetric halves. Each half is organized into 24 azimuthal modules called wedges (see figure 2.5). Each wedge consists of a number of projective towers pointing to the center of the calorimeter. The segmentation in $\eta$ and $\phi$ is: $\Delta \eta \times \Delta \phi=0.1 \times 15^{\circ}$. The process which allows to make energy measurements is the following: the primary charged particle loses its energy by bremsstrahlung and pair production, which produce an electromagnetic shower. The CEM measures the total energy of $e^{ \pm}$and $\gamma$ by sampling the energy released by the electromagnetic shower and a (minor) fraction of the hadronic energy. Charged particles passing through the scintillator produce light. This light is then collected by wave length shifter plastics and transmitted to a phototube by means of light-guides. An electromagnetic shower reaches its maximum at 6-7 radiation lengths $\left(\chi_{0}\right)$ after which it decays exponentially. In the CEM the shower is sampled up to about 18 radiation lengths. The main error in determining the energy of the primary incident $e^{ \pm}$is due to the statistical fluctuation in the number $N_{e^{ \pm}}$ of $e^{ \pm}$which belongs to the shower and passes through the scintillator: $\sigma_{E} \propto \sqrt{N}_{e^{ \pm}}$. Since $N_{e^{ \pm}}$is proportional to the energy $\mathbf{E}$ of the incident $e^{ \pm}$, the uncertainty becomes: $\sigma_{E} \propto \sqrt{E} \rightarrow$ $\frac{\sigma_{E}}{E} \propto \frac{1}{\sqrt{E}}$. For the CEM $\frac{\sigma_{E}}{E}=\frac{13.5 \%}{\sqrt{E}} \oplus 2 . \%$, where $\mathrm{E}$ is given in $\mathrm{GeV}$. The second term added in quadrature comes from a systematic source and is primarily due to the difference in the energy calibration of different towers. Inside the CEM, at about 6 radiation lengths, proportional wire chambers (CES) have been installed. The wires (anodes) provide the information on the $r$ and $\phi$ coordinates, while the strips (cathodes) give the shower $z$ position. The CES have a main role in the separation of $e^{ \pm}$and $\gamma$ fro $\pi^{0} \rightarrow \gamma \gamma$. In 1992 proportional chambers (CPR, central pre-radiators) were inserted outside the magnetic coil and in front of the wedges. They detect photon conversions and improve the separation power between $\pi^{\circ}$ and $\gamma$.

The CHA works in a similar way to the CEM. Incident hadrons lose most of their energy 


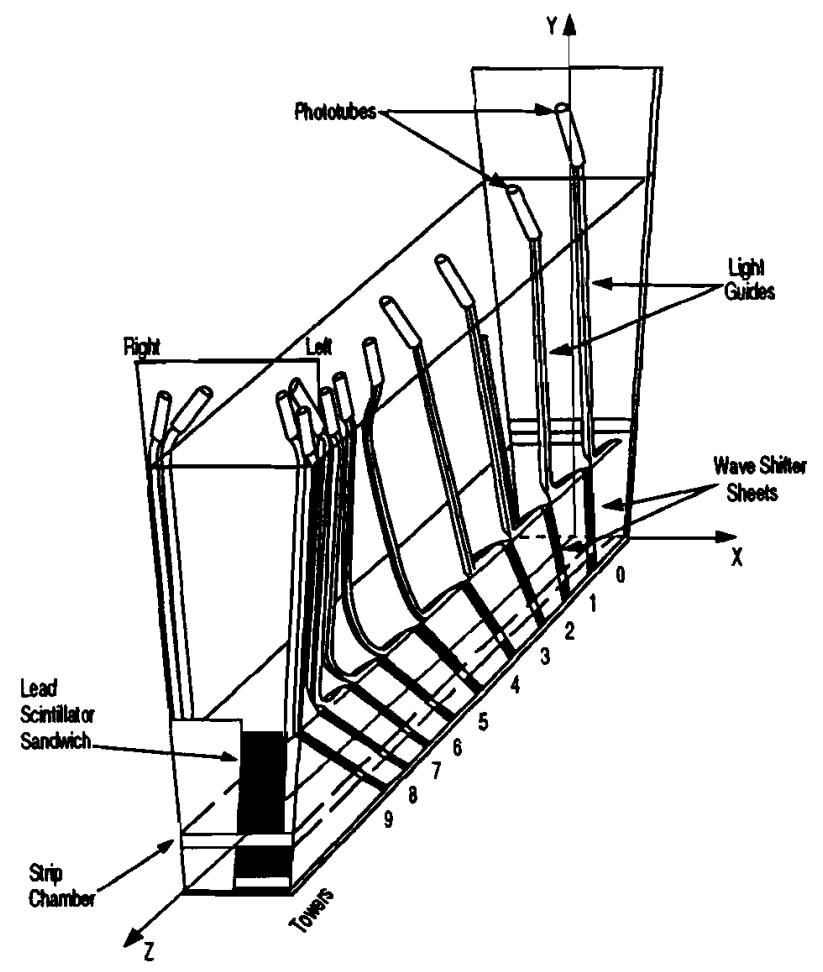

Figure 2.5: Schematic of a CEM module. The system which collects and tranmits the signal to the photomultipliers is also shown.

developing an hadronic shower. In spite of the good luminosity, statistical fluctuations in the energy measurement are larger than in the CEM. This is due to the unavoidable fluctuations which occur in the fragmentation process as well as in the hadronic nuclear excitations. The CDF CHA extends for about a 5 interaction length and has an energy resolution for incident pions of $\frac{\sigma_{E}}{E}=\frac{75 \%}{\sqrt{E}} \oplus 3 \%$.

\section{Forward calorimetry}

In the intermediate angle region ("plug", at $1.1 \leq|\eta| \leq 2.2)$ and in the forward region (2.2 $\leq$ $|\eta| \leq 4.2)$ gas proportional chambers with an argon-ethan mixture are used as active medium. The resolution of the electromagnetica part is $\sim 4 \%$ for $50 \mathrm{GeV}$. The resolution of the hadronic part is $\sim 20 \%$ for $50 \mathrm{GeV}$ pions. Table 2.1 summarizes the main properties of CDF calorimeters. 


\begin{tabular}{|l|c|c|c|}
\hline System & $\eta$ range & Energy Resolution & Thickness \\
& & & \\
\hline CEM[57] & $|\eta|<1.1$ & $13.5 \% / \sqrt{E_{T}} \oplus 2 \%$ & $18 \chi_{0}$ \\
PEM[58] & $1.1<|\eta|<2.4$ & $28 \% / \sqrt{E} \oplus 2 \%$ & $18-21 \chi_{0}$ \\
FEM[59] & $2.2<|\eta|<4.2$ & $25 \% / \sqrt{E} \oplus 2 \%$ & $25 \chi_{0}$ \\
CHA[60] & $|\eta|<1.3$ & $75 \% / \sqrt{E_{T}} \oplus 3 \%$ & $4.5 \lambda_{0}$ \\
PHA[61] & $1.3<|\eta|<2.4$ & $90 \% / \sqrt{E_{T}} \oplus 4 \%$ & $5.7 \lambda_{0}$ \\
FHA[62] & $2.4<|\eta|<4.2$ & $130 \% / \sqrt{E_{T}} \oplus 4 \%$ & $7.7 \lambda_{0}$ \\
\hline
\end{tabular}

Table 2.1: Main properties of CDF calorimeters. The symbol $\oplus$ indicates that the constatnt term is added in quadrature. Energy resolutions for the electromagnetic (hadronic) calorimeters are given for incident $e$ and $\gamma$ (isolated pions). Thickness is given in radiation lengths $\chi_{0}$ and interaction lengths for the electromagnetic and hadronic calorimeters respectively.

\subsubsection{Muon detectors}

The muon central detector (CMU)[63] is located on the back of the central hadronic calorimeter, outside each wedge, at a radial distance of $3.5 \mathrm{~m}$ from the beam axis. It consists of 48 modules called muon chambers, two for each wedge. Each module covers about $12.6^{\circ}$ in $\phi$ (with an uninstrumented region of about $1.2^{\circ}$ at the ends of a wedge). The muon chambers are proportional drift chambers which measure charged particle tracks. The central calorimeters act as a hadron absorber (4.9 interaction lengths) in front of the chambers. The chambers can be reached by muons with $P_{t}>1.5 \mathrm{GeV} / \mathrm{c}$. Each chamber consists of four layers that are segmented in $\phi$ to form three towers of $4.2^{\circ}$ each. Figure 2.6 shows the geometry of one of these towers, which contains 16 rectangular drift cells. Four sense wires, one for each layer, form a muon tower. The sense wires are read at both ends, to measure the $z$ position with a precision of $1.2 \mathrm{~mm}$, using the charge division technique. Tracks are reconstructed in the $r-\phi$ plane using drift time information. If there is a signal in at least three of the four chamber layers a track segment called "stub" is reconstructed. If this segment is associated to a CTC track, then one has a muon candidate. The CMU system covers $84 \%$ of the $|\eta|<0.6$ region $^{1}$. In 1992 , a $60 \mathrm{~cm}$ layer of steel was added behind this apparatus, followed by four layers of drift chambers (CMP)[64]. This upgrade has been done in order to improve $\mu$ detection. In

\footnotetext{
${ }^{1}$ The $16 \%$ loss in acceptance is due to the separation $\left(2.4^{\circ}\right)$ between the wedges and to the separation of the two calorimeter arches at $\eta=0$.
} 


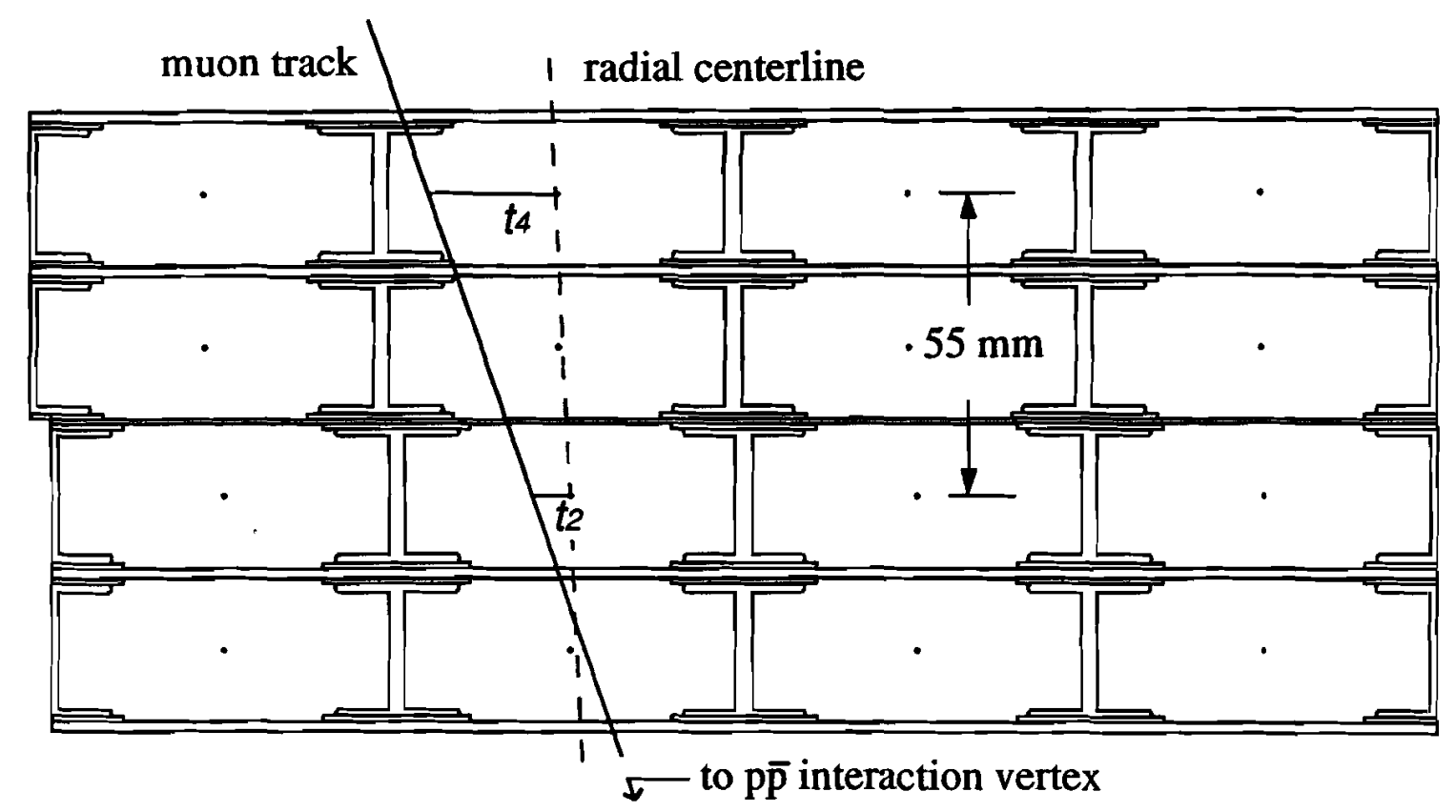

Figure 2.6: Layout of a muon tower, consisting of 16 rectangular drift chambers on 4 planes. The drift times $t_{i}$ and the track angle are also shown.

fact it reduces the background due to those hadrons which escape from the calorimeter and therefore it improves the trigger and muon identification. For the 1992 run, the coverage of the CMU+CMP system was extended through the addition of another muon detector. This new detector, consisting of drift chambers (CMX[65]) and layers of scintillators for the trigger (CSX[66]), increased the acceptance to $|\eta|<1$. Table 2.2 summarizes the main properties of the three components of the muon detectors.

\begin{tabular}{|l|lll|}
\hline Property & CMU & CMP & CMX \\
\hline \hline$|\eta|$ coverage & {$[\overline{0,0.63]}$} & {$[0,0.60]$} & {$[0.62,1.0]$} \\
$\phi$ coverage & $84 \%$ & $84 \%$ & $75 \%$ \\
Minimum detectable $\boldsymbol{P}_{\boldsymbol{t}}$ & $1.6 \mathrm{GeV} / c$ & $3.0 \mathrm{GeV} / c$ & $1.7 \mathrm{GeV} / c$ \\
\hline
\end{tabular}

Table 2.2: Summary of the central muon detectors. 


\subsubsection{Trigger}

For the top search in decay channels with leptons it is very important to have a highly efficient trigger for high $\mathrm{P}_{t}$ muons and electrons. In particular, for the analysis developed in this thesis events with central $\left(|\eta|<1\right.$.) electrons and muons, with $\mathbf{P}_{t}>20 \mathrm{GeV} / \mathrm{c}$ have been used. These events have been selected by means of inclusive electron and muon triggers. These triggers use the information coming from the tracking system, from the calorimeter and from the muon chambers as described in the following. The trigger system for the 1992-93 CDF run is a three level system[67]. This trigger has been designed in order to reduce the event candidate rate so that it is possible to record them (1-2 Hz). Each event is a logical OR of a number of triggers which select events with electrons, muons, jets and other parameters of interest.

- The first level of trigger uses fast outputs from the muon chambers and from the calorimeters, for electrons and jets. The information coming from the CEM and the CHA is summed into towers of $\Delta \eta \times \Delta \phi=0.2 \times 15^{\circ}$. A single trigger tower with $\mathrm{E}_{t}>6 \mathrm{GeV}$ in the $\mathrm{CEM}$ or $\mathrm{E}_{t}>8 \mathrm{GeV}$ in the CHA is required for the electrons. The muon trigger requires instead a CMU track with $P_{t}>6 \mathrm{GeV} / c$ coincidental with hits in the CMP or a CMX track with $P_{t}>10 \mathrm{GeV} / \mathrm{c}$ coincidental with scintillator hits on both sides of the chambers. The CMX inclusive muon trigger was fully functional only in the second part of the run (see Appendix A). In order to preserve the sensitivity to CMX muons from top decays, the level 1 single tower calorimeter trigger was required in addition to the CMX muon candidate. This trigger has been present for $83 \%$ of the run.

- The second level of trigger uses the calorimeter and CTC information in a more sophisticated way. First, a list of calorimeter clusters is provided. For each cluster the $\mathrm{E}_{t}$ as well as average $\phi$ and $\eta$ are provided. This information is then combined with a list of $r-\phi$ tracks found by a fast processor (Central Fast Tracker or CFT) which uses timing information from the CTC as input. The CFT resolution and its efficiency are: $\delta P_{t} / P_{t} \simeq 0.035 \times \mathrm{P}_{t}$ and $\epsilon=(93.5$ $\pm 0.3) \%$ for isolated tracks with $P_{t}>10 \mathrm{GeV} / \mathrm{c}$. At this level muon track segment information from the CMU, CMP and CMX is also available. CFT tracks which point to electromagnetic clusters identify electron candidates. CFT tracks which point to tracks in the muon chambers 
identify muon candidates.

In particular, for the electrons a cluster in the CEM with $E_{t}>9 \mathrm{GeV}$ together with an associated CTC track with $P_{t}>9.2 \mathrm{GeV} / \mathrm{c}$ are required. An electromagnetic cluster is constructed as a set of contiguous CEM trigger towers with $E_{t}>7 \mathrm{GeV}$ including at least one "seed" tower with $\mathrm{E}_{t}>9 \mathrm{GeV}$. The ratio of hadronic to electromagnetic energy in the cluster must be less than 12.5\%. For muons it is required that the extrapolation of a CFT track with $P_{t}>9.2$ $\mathrm{GeV} / \mathrm{c}$ match with a track in the muon chamber.

- The third level of trigger is based on an algorithm which uses a system of Silicon Graphics Multicpu Power Servers which, combined together, are able to process data at the frequency of $20 \mathrm{~Hz}$. Then there are fortran algorithms for the event reconstruction. In this last stage, the longest process is the tridimensional reconstruction of the CTC tracks. For the electrons, the third level requires that the cluster reconstructed in the calorimeters has $\mathrm{E}_{t}>18 \mathrm{GeV}$ and that there is a track in the CTC which points in the cluster direction. The detection efficiency for electrons with $20<\mathrm{E}_{t}<150 \mathrm{GeV}$, is $(92.8 \pm 0.2) \%$. For the muons, one requires a track in the CTC with $P_{t}>18 \mathrm{GeV} / c$ and with a distance from the track measured in the muon chambers of less then $10 \mathrm{~cm}$ in $r * \phi$. In addition, the energy deposition in the CHA must be less than $6 \mathrm{GeV}$. For the $\mathrm{CMU}(\mathrm{CMX})$ trigger, an efficiency of $86.8 \pm 1.9 \% .(54.4 \pm 5.5 \%)$ was measured for muons with $\mathrm{P}_{t}>20 \mathrm{GeV} / \mathrm{c}$. 


\section{Chapter 3}

\section{Event Selection and Reconstruction}

In order to look for top in the $\mathrm{W}+$ jets channel, one starts by selecting an inclusive $\mathrm{W}$ sample of good quality, with little contamination by non-W events. W's are identified from the products of their leptonic decay: $\mathrm{W} \rightarrow e / \mu+\nu$.

\subsection{Electron Identification}

Electrons deposit most of their energy in the electromagnetic calorimeter, and the electromagnetic cascade which they produce is normally contained in few calorimeter towers. Pointing towards this electromagnetic cluster is a CTC track with momentum approximatively equal to the energy found in the calorimeter, within the measurement accuracy. The position of the electromagnetic shower is measured by proportional chambers (CES), within the calorimeter, and the charged track must match this position.

Hadronic jets have a different signature. They deposit energy in both the electromagnetic and hadronic calorimeters, over a larger area in $\eta-\phi$. Normally, hadronic jets show many charged tracks in the CTC. Due to fluctuations in the parton fragmentation process and in the shower development, it can sometimes happen that a hadronic jet looks very similar to an electron. For example, if most of the jet energy is carried by $\pi^{0}$ or $\dot{\eta}$, this energy will be released as an electromagnetic shower in the calorimeter. In addition there may be a charged particle $\left(\pi^{ \pm}\right)$ in the jet which happens to point to the electromagnetic cluster.

For this analysis electrons in the central region $(|\eta|<1)$ have been used. Electron candidates 
are required to have a track in the CTC which matches the position of an electromagnetic cluster. Such a cluster gets reconstructed starting from a tower with $E_{t}>3 \mathrm{GeV}$ and including the two nearest towers in pseudorapidity. It therefore extends for 3 towers in $\eta(\Delta \eta=0.3)$ and one tower in azimuth $\left(\Delta \phi=15^{\circ}\right)$. The electron candidates need to have a well measured calorimeter energy. For that reason one requires that they are in 'fiducial regions' of the calorimeter, where there are no cracks (as for example the space between the calorimeter wedges). This volume covers about $84 \%$ of the solid angle for $|\eta|<1$. To improve the electron/hadron separation the following is also required:

a) An electromagnetic fraction for the calorimeter energy deposit $<0.05$;

b) A ratio of the calorimeter energy (E) over momentum of the track in the CTC (P) $\mathrm{E} / \mathrm{P}<1.5$

c) A lateral cluster profile that is consistent with what has been observed in the test beam. This consistency is measured in terms of a variable called $\mathrm{L}_{S H R}$ : one requires $\mathrm{L}_{S H R}<0.2$;

d) A distance in $r * \phi(z)$ between the extrapolated track and the position of the electromagnetic shower measured by the CES $<1.5(<3$.) $\mathrm{cm}$;

e) A $\chi^{2}$ measuring the consistency between the profile of the shower provided by proportional chambers and by test beam measurements: $\chi^{2}<10$;

f) A distance $z<5 \mathrm{~cm}$ between the interaction vertex and the reconstructed track.

The electron identification has an efficiency of $(84 \pm 2) \%$.

\subsection{Muon Identification}

Muons travelling through the detector first leave a track in the CTC, and then deposit an amount of energy in the calorimeter typical of a minimum ionizing particle. Outside of the calorimeter the muons leave a short track (stub) in the muon chambers. This stub must be consistent with the track extrapolation from the CTC, within the measurement errors due to 
Coulomb scattering. Their signature is therefore much different from that of an hadronic jet. Still, the inclusive muon sample will have several sources of background. For example it can happen that a hadron does not undergo a strong interaction within the calorimeter material, and in this case it is not possible to distinguish it from a real muon ${ }^{1}$. It also can happen that $\pi$ 's or K's decay to a muon within the volume of the CTC, or inside the calorimeter. Muons from background usually have a lower $P_{t}$ than muons from a $W$ decay. Therefore they undergo more Coulomb scattering and their track extrapolation will on average match the track in the muon chambers less well. Finally, some of the particles which constitute the jet may form a shower at the end of the calorimeter and part of the shower can leak out of the calorimeter, producing hits in the muon chambers. In this way high $E_{t}$ jets can fake muons as well. In most of these cases the matching between the CTC muon candidate track to the muon chamber hit will be poor.

For this analysis muons in the central rapidity $(|\eta|<1)$ region are used, and a stub matching the CTC track extrapolation in either the CMU, CMP or CMX system is required. In order to identify the muons the following cuts are applied :

a) A track in the muon chambers CMU, or CMU and CMP, or CMX;

b) An energy in the calorimeter which is compatible with minimum ionization: $<2 \mathrm{GeV}$ in the CEM, $<6 \mathrm{GeV}$ in the CHA;

c) An impact parameter (smallest distance between track and beam line) $<3 \mathrm{~mm}$;

d) A distance of the track from the interaction vertex in the beam direction of $|z|<5 \mathrm{~cm}$;

e) A distance in $r * \phi$ between CTC track extrapolation and muon chamber hit of $<2 \mathrm{~cm}$ for the CMU system or $<5 \mathrm{~cm}$ for the CMP or CMX system.

The muon identification is $(90.6 \pm 1.4) \%$ efficient.

\footnotetext{
${ }^{1}$ The CDF calorimeter consists of about 5 interaction lengths of material, and therefore the probability that a hadron reaches the muon chambers without interacting is about $e^{-5}=0.0067$.
} 


\subsection{Jets}

The fragmentation of a parton produces a group of particles which are approximately collinear to the direction of the original parton. However, the transverse spread of these particles causes the calorimeter cluster to be much broader than that from an electron, since it extends typically over many towers. The CDF jet finding algorithm uses a fixed cone in $R\left(R=\sqrt{\Delta \eta^{2}+\Delta \phi^{2}}\right)^{2}$ to define a jet. One starts from a so-called "seed tower" which must have $\mathrm{E}_{t}>1 \mathrm{GeV}$. Then all towers with $\mathrm{E}_{t}>0.1 \mathrm{GeV}$ in a cone of radius $\mathrm{R}$ around the seed tower are added. The center of this energy cluster is then calculated, and around this center a new cone is built. This process gets repeated until the list of the towers included does not change anymore. If two clusters are close to each other and have more than $50 \%$ of the energy in common, they are merged. If they have energy in common, but less than $50 \%$, the towers get assigned to the nearest cluster.

In this thesis we are using a jet reconstruction cone of $\mathrm{R}=0.4$

\section{4 $\mathbf{E}_{t}$ Jet Correction}

If a jet hits a region of the calorimeter where different detector components are connected (that is, an uninstrumented area), its energy usually gets underestimated. One also has to consider that the calorimeter does not have a linear energy response for low momentum hadrons. This affects the jet measurement $[68,69]$. Some of the jet energy will end up outside of the jet reconstruction cone. In addition, some of the energy inside the cone will be from the underlying event and not from the original parton. One has to correct the observed jet energy for these effects. These corrections are of particular importance in a study which makes use of the $\mathrm{E}_{t}($ jet $)$ spectra.

For the central region of the calorimeter one can compare the observed energy with the momentum as it is measured in the CTC. In this way one can calibrate the calorimeter response for hadrons of different energies. One also knows the kinematical characteristics of hadrons

\footnotetext{
${ }^{2} \Delta \eta$ is the cone size in pseudorapidity and $\Delta \phi$ is the cone width in the arimuthal angle.
} 
which are produced in parton fragmentation processes. These two pieces of information give the calorimeter response to partons of different energies. However, the correction needs to be found for all other regions of the calorimeter too. Based on energy conservation in two-jet events one can obtain a jet energy correction map extending over the whole rapidity range[70]. One of the two jets is required to be in the central part of the calorimeter, where the response is known, and is used as reference. The other one can be anywhere. In figure 3.1 we show the relative corrections for two different intervals in uncorrected jet $E_{t}\left(E_{t}^{n c}\right): 12<E_{t}^{n c}<20$ $\mathrm{GeV}$ (solid line) and $30 \mathrm{GeV}<\mathrm{E}_{t}^{n c}<60 \mathrm{GeV}$ (dotted line). The jet correction factor becomes larger in regions of rapidity where there are discontinuities in the calorimeter, for example between the central calorimeter and the plug calorimeter, at rapidities of about 1.1 and -1.1 .

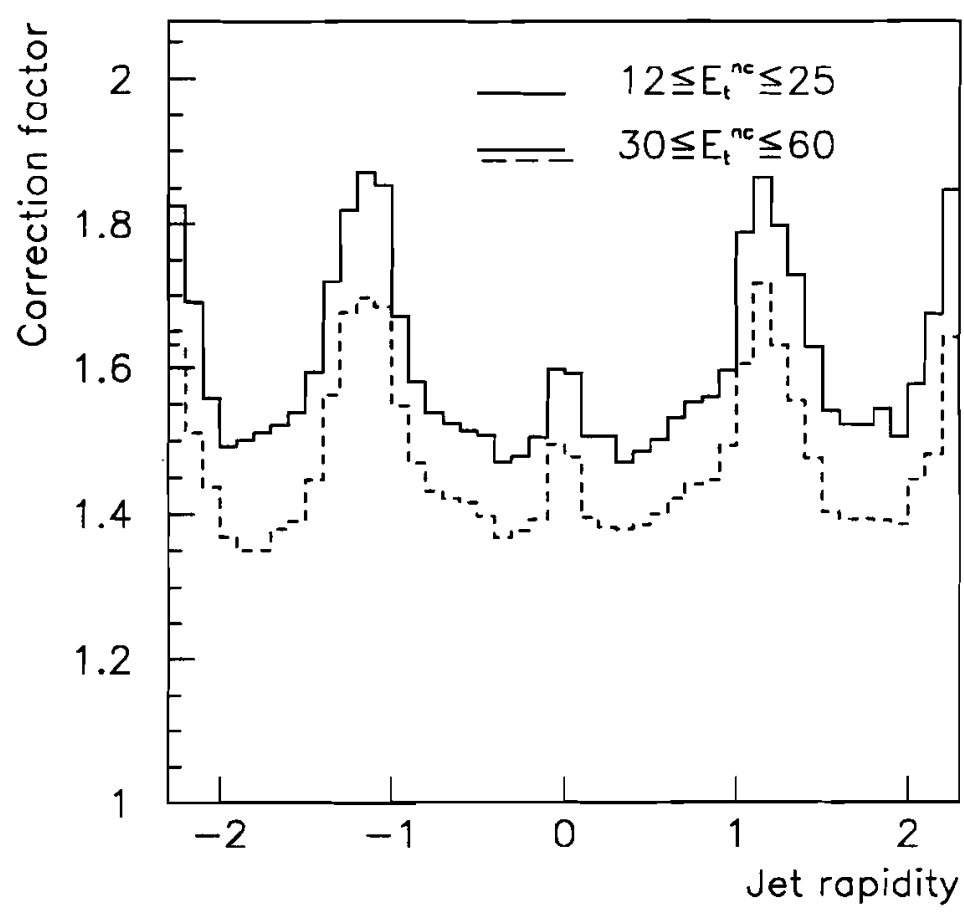

Figure 3.1: Relative jet corrections in two intervals of $E_{t}^{\text {nc }}: 12<E_{t}^{\text {nc }}<20 \mathrm{GeV}$ (solid line) and $30<E_{t}^{n c}<60 \mathrm{GeV}$ (dotted line). 


\subsection{Missing Energy}

Neutrinos do not interact with any detector component and cannot therefore be directly observed. One can indirectly measure them by making use of the conservation of the total $\mathrm{E}_{t}$ in each event. The missing transverse energy (often simply referred to as "missing energy") $\boldsymbol{E}_{t}$ is defined as :

$$
\overrightarrow{\boldsymbol{E}}_{t}=-\sum_{i} \vec{E}_{t}^{i}
$$

where $\vec{E}_{t}^{i}$ is the vector of the energy deposit in the calorimeter towers, and the sum is over all towers at $|\eta|<3.6^{3}$ with an energy above a certain threshold, depending on the part of the calorimeter (e.g.: $100 \mathrm{MeV}$ in the CEM). Minimum ionizing particles of high momentum, like muons, deposit only a small part of their energy in the calorimeter. For this reason in events with muon candidates the $\boldsymbol{F}_{t}$ gets corrected by subtracting vectorially the energy in the tower hit by the muon and adding the $P_{t}$ of the muon as measured in the CTC.

\subsection{Missing Energy Correction}

The measurement of $\boldsymbol{F}_{t}$ requires that one has measured correctly all the energy deposited in the detector. A mismeasurement of $F_{t}$ can increase the background in our W sample. Part of the energy deposited in the detector is in calorimeter clusters, and the rest is distributed in the detector without forming any clusters (unclustered energy). We know that the jets need to be corrected because their observed energy does not correspond to the real one. In order to get the best possible measurement of $\boldsymbol{E}_{t}$, one also needs to study whether and how the unclustered energy needs to be corrected. For the energy correction studies events with a lepton candidate and at least two jets of $E_{t}>15 \mathrm{GeV}$ have been used. First a sample of photon conversions [71] from the process $\gamma \rightarrow e^{+} e^{-}$has been selected. In these photon conversions, there should be no high- $\mathrm{E}_{t}$ neutrinos. A significant $\boldsymbol{E}_{t}$ in these events indicates an incorrect calorimeter energy measurement. To evaluate the quality of the missing energy correction the fraction of conversion events which have $M_{\text {trans }}>40 \mathrm{GeV}^{2}$ has been used, since this cut is

\footnotetext{
${ }^{3}$ The interval in $\eta$ is restricted because part of the FHA is shielded by the focusing magnets of the Tevatron.
} 
commonly used to identify $\mathrm{W} \rightarrow l \nu$. In table 3.1 we show this fraction for various correction procedures :

- jet energies have been calculated with and without out-of-cone corrections, for two different jet reconstruction cone sizes $(R=0.4$ and $R=0.7)$;

- clusters have been formed starting from two different thresholds in $\mathrm{E}_{t}^{n c}$ (uncorrected $\mathrm{E}_{t}$ ) $(10$ or $5 \mathrm{GeV})$;

- several different correction factors for the unclustered energy (1., 1.3 and 1.6) have been used.

Before any correction, the fraction of events with $\mathbf{M}_{\text {trans }}>40 \mathrm{GeV} / \mathrm{c}^{2}$ is $33 \%$. If corrections are applied to all the jets formed by clusters with $\mathrm{E}_{t}^{n c}>10 \mathrm{GeV}$ (not including out-of-cone correction or unclustered energy correction) one gets a fraction of $18-19 \%$, for both cone sizes. This is already a clear improvement. If one corrects all clusters with $\mathrm{E}_{t}^{n c}>5 \mathrm{GeV}$ the results are slightly better. Applying the out-of-cone corrections brings an improvement. However, there is a logical difficulty in correcting the unclustered energy and in applying at the same time the out-of-cone corrections. This difficulty is avoided if one uses a cluster threshold of $5 \mathrm{GeV}$, a jet reconstruction cone of $R=0.4$, and no corrections to the unclustered energy. This procedure gives a good background rejection. We will always apply it in the following. Figure 3.2 (a) shows the distribution in $\mathbf{M}_{\text {trans }}$ before (solid line) and after (dotted line) applying the jet corrections to correct the missing energy for events with a high quality electron. Figure 3.2 (b) shows the same distributions for the conversion events. The figures demonstrate why one needs to correct the missing energy. To have one more check a sample of non-isolated electrons has also been selected (Iso $>0.1$, see subsection 3.7 for the definition of Iso). This sample should be dominated by fake leptons and by leptons from beauty quark decays. Neither fake leptons nor $b$ decays should have large missing energy. The corresponding $\mathbf{M}_{\text {trans }}$ distribution is shown in figure 3.3. Again, the solid line shows the spectrum before the missing energy correction, the dotted line after. One sees that it is possible to reduce this kind of background by applying a proper $\boldsymbol{E}_{\boldsymbol{t}}$ correction. 


\begin{tabular}{|l|c|c|c|}
\hline $\begin{array}{l}\text { correction factor } \\
\text { for } \mathrm{E}_{\boldsymbol{t}} \text { unclustered }\end{array}$ & 1. & 1.3 & 1.6 \\
\hline \hline Cone 0.7, $\mathbf{E}_{t}^{n c}$ cluster> 10 GeV & & & \\
& & & \\
out-of-cone:No & $19 . \%$ & $15 . \%$ & $17 . \%$ \\
out-of-cone:Yes & $17 . \%$ & $11 . \%$ & $12 . \%$ \\
\hline Cone 0.4, $\mathbf{E}_{t}^{n c}$ cluster> 10 GeV & & & \\
& & & \\
out-of-cone:No & $18 . \%$ & $18 . \%$ & $18 . \%$ \\
out-of-cone:Yes & $12 . \%$ & $8 . \%$ & $8 . \%$ \\
\hline Cone 0.4, $\mathbf{E}_{t}^{n c}$ cluster> 5 GeV & & & \\
& & & \\
out-of-cone:No & $16 . \%$ & $17 . \%$ & $17 . \%$ \\
out-of-cone:Yes & $8 . \%$ & $7 . \%$ & $8 . \%$ \\
\hline
\end{tabular}

Table 3.1: Fraction of photon conversion events with $M_{\text {trans }}>40 \mathrm{GeV} / \mathrm{c}^{2}$, after applying various energy correction procedures. Before any energy correction, the fraction is $33 \%$.

\subsection{W Identification}

To identify and select W's one requires that the interaction vertex be closer than $60 \mathrm{~cm}$ from the center of the detector. In addition, one applies the following cuts[72]:

a) The lepton needs to be of high quality according to the criteria described in subsections 3.1 and 3.2. The lepton must be isolated: that means there must be little energy in the calorimeter towers neighboring the lepton. For electrons:

$$
I s o=\frac{E_{t}^{c}-E_{t}}{E_{t}}<0.1
$$

where $\mathrm{E}_{t}^{c}$ is the transverse energy in a cone of 0.4 around the electron (excluding the electron cluster itself), and $E_{t}$ is the transverse energy of the electron.

For muons:

$$
I s o_{\mu}=\frac{E_{t}^{c}-E_{t}^{c 2}}{P_{t}(\mu)}<0.1
$$

where $E_{t}^{c}\left(E_{t}^{c 2}\right)$ is the transverse energy in a cone of $R=0.4(0.13)$ around the muon.

These isolation cuts reduce the background from quarks (which create jets, and therefore give non-isolated leptons). 

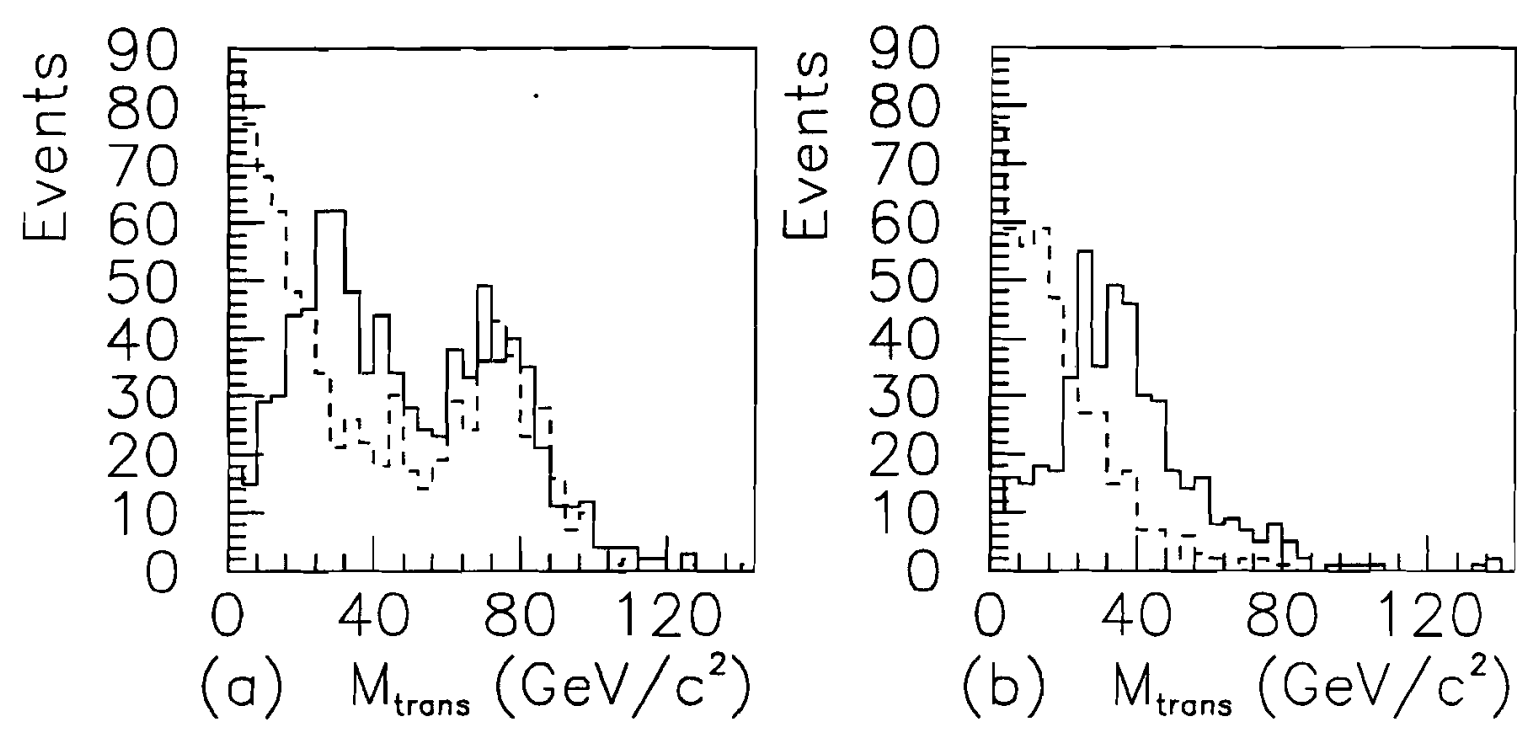

Figure 3.2: (a) $M_{\text {trans }}$ distribution for events with an isolated lepton of good quality before (solid line) and after (dotted line) correcting the missing energy. (b) The same distributions for events from photon conversions.

b) The lepton has to have $P_{t}>20 \mathrm{GeV} /$ c. Kinematics of $\mathrm{W}$ decay results in a maximum of $\mathrm{P}_{t}$ (lepton) around $40 \mathrm{GeV} / \mathrm{c}$ (Jacobian peak).

c) Require $Z_{t}>25 \mathrm{GeV}$ to reduce backgrounds from $b \bar{b}[72]$.

d) Require $\mathbf{M}_{\text {trans }}>40 \mathrm{GeV} / \mathrm{c}^{2}$ for the lepton-neutrino system (compare figure 3.2 (a)).

The remaining $\mathrm{W}$ sample still contains some background. This background consists of :

1.) $Z^{0}$ events which passed the $\boldsymbol{E}_{t}$ cut;

2.) Events where the electron comes from a photon conversion;

3.) Events where the lepton candidate passed all the electron quality requirements, but is not really an electron;

4.) Events where a real lepton passed the isolation cuts, but is not from a W decay. 


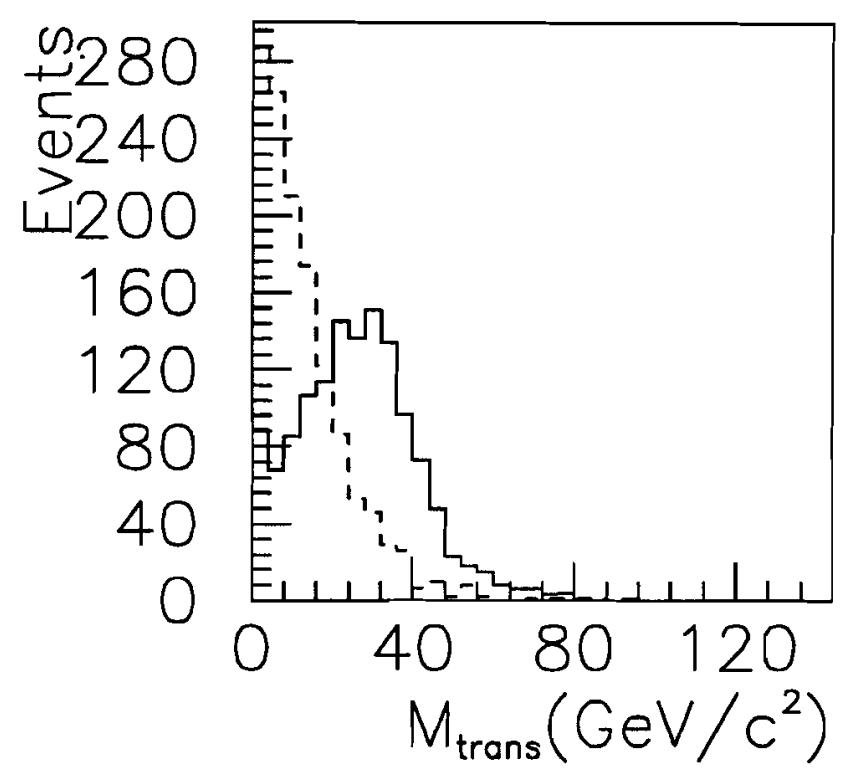

Figure 3.3: $M_{\text {trans }}$ distributions for events with a non-isolated lepton before correcting the missing energy (solid line) and after applying the corrections (dotted line).

Points 3.) and 4.) will be discussed in more detail in Chapter 5.

In order to reduce the background from Z's, one can look for a second lepton candidate in the events. To efficiently find the second lepton, we simply look for an additional track with $\mathrm{P}_{t}>15 \mathrm{GeV} / \mathrm{c}$ which is isolated in the CTC[73]. The track is defined to be isolated if the sum of the transverse momenta of all other tracks in a cone of $R=0.4$ around this track is less then 0.1 times the track $P_{t}$. A study of QCD jets has shown that only about $1 \%$ of the jets contains a high pt isolated track[73]. The di-lepton candidates which are defined in this way are removed from the sample.

For point 2.) we can make use of the tracking system. We reject events where the electron track from the CTC does not correspond to a track in the VTX detector (indicating a conversion in the outer part of the VTX), or where there is a nearby track of opposite charge forming a low invariant mass together with the electron candidate. Studies using data and Montecarlos 
have shown that $(88 \pm 4) \%$ of the conversions can be removed in this way.

Finally one requires that the $\mathrm{W}$ events activate a lepton trigger. In figure 3.4 we show an example for a $W+$ jet event in the CDF detector. The projection in the $r-\phi$ plane shows only

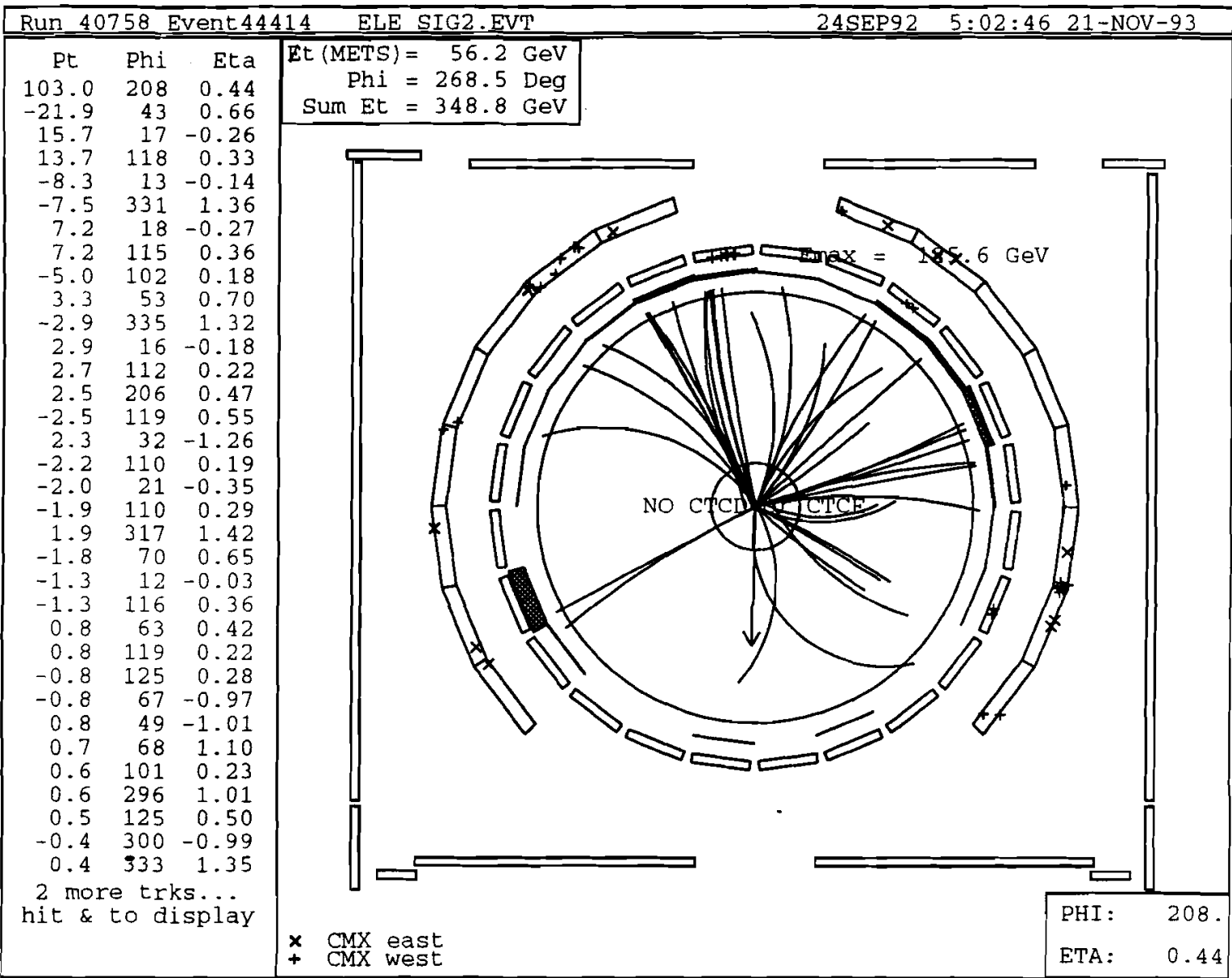

Figure 3.4: Reconstruction of a $W$ event with jets.

the central part of the detector and the figure is not to scale (for example the calorimeters are much reduced in size). The beam axis is in the center. We see tracks emerging from the vertex, which get bent by the magnetic field according to their electric charge and their transverse momentum. Where the tracks hit the calorimeter we see energy deposits, shown as small rectangles of different heights. Downwards, on the left side, we see a track which is bent very little, that means of high $P_{t}$, which deposits a lot of energy in one calorimeter 
cell. This is the electron candidate. Most of the other tracks appear in groups and form the hadronic jets of this event. When one sums up all the transverse energy, the resulting $E_{t}$ is quite different from zero, which indicates the presence of a neutrino.

This event with a W and jets is also a typical top candidate. We will meet it again later (Chapter 5) and still later (Chapter 6) we will find that it can be reconstructed as a $t \bar{t}$ event with a top mass of about $180 \mathrm{GeV} / \mathrm{c}^{2}$. One also sees a track very close to the electron. This track has the same charge as the electron, but has a much lower $P_{t}$. There is also a third track very close to these two, with opposite charge. It is not shown in the figure because of its low momentum. The high momentum electron comes from the primary interaction vertex, the two low $P_{t}$ tracks however both come from a point on the electron track, a few centimeters away from the vertex. The most likely interpretation is that the high momentum electron has radiated a photon when it traversed the material of the detector. This photon converted to a $e^{+} e^{-}$pair. Effects of this kinds are well studied at CDF in the context of the $\mathrm{Z}^{0}$ mass measurement[74], which is performed using both the CTC and the calorimeter information.

In this event display the SVX detector is not visible. Otherwise one would see very good evidence for a secondary vertex in one of the jets, which indicates the presence of a $b$ quark. We see that CDF is able to identify particles with masses between $0 \mathrm{GeV} / \mathrm{c}^{2}$ (photon), 5 $\mathrm{GeV} / \mathrm{c}^{2}$ ( $b$ quark), $80 \mathrm{GeV} / \mathrm{c}^{2}(\mathrm{~W})$ and about $180 \mathrm{GeV} / \mathrm{c}^{2}$ (top candidate). And all this in one event! 


\section{Chapter 4}

\section{Montecarlo Simulations}

\subsection{VECBOS}

To simulate W+jet QCD events, the VECBOS Montecarlo has been used. VECBOS has been introduced in Chapter 1 , and it is based on the calculations described in subsection 1.4.1. VECBOS calculates the matrix element (ME) for each event, and gives the event a corresponding weight. The event weight is determined by the product of the ME times the volume of the phase space hypercube in which the event lies.

In the phase space region where the $\mathrm{ME}$ is large, the weight can have very large values. This is the case, as an example, for $\mathrm{W}+\mathrm{jet}$ events with low $\mathrm{E}_{t}$ or forward emitted partons.

Inside the phase space allowed by the kinematical cuts, the $M E$ is included between a maximum and a minimum value. These two values can differ by several orders of magnitude. The difference between the smallest and the largest possible value for the ME increases with the jet multiplicity.

One can have a very broad distribution of event weights, which means large statistical errors. It is therefore profitable to narrow the weight distributions in order to obtain reasonable statistical errors without generating a huge amount of Montecarlo events.

For the events to have the same weight, the density of Montecarlo events in the phase space should be proportional to the ME. To arrive at such a result, it is necessary to subdivide the phase space in small cells. The volume of these cells must be inversely proportional to the dimension of the corresponding ME. This is difficult to achieve especially in the case of 
multidimensional phase space. VECBOS can only subdivide the initial phase space (twodimensional), identified by the momenta of the initial partons.

As a first step VECBOS divides the initial state phase space in bins of equal dimensions, and generates the same number of events in each bin. The density of the events is then increased by reducing the bin area where the ME is larger. Still, in this two-dimensional phase space VECBOS cannot correctly take into account the correlations between the two variables. The final state is populated randomly.

As a result the weight distributions are broad, in particular for $\mathrm{W}+4$ jet events. It is therefore necessary to produce a large number of events in order to reduce the statistical errors.

For the work described in this thesis $10^{6} \mathrm{~W}+3$ parton and $5 \cdot 10^{5} \mathrm{~W}+4$ parton events have been generated. The distribution in the logarithm of the weights for VECBOS $W+3$ jet events is shown in figure 4.1. As one can see, the weights are significantly different for different events. For a Montecarlo sample of weighted events one can adopt the "unweighting" procedure, which allows removal of the weight from the events. This procedure selects events randomly from the sample, with a probability proportional to the weight of the events. For each event a random number between 0 and the maximum weight in the sample is generated. If the event weight is larger than this number the event is kept, otherwise it is rejected. In this way one can reduce his original sample, to a smallexnumber of events. This reduced sample does not need a large amount of disc space and can be processed more easily.

This procedure has not been applied in our analysis, since it is suspected of introducing an additional statistical error (the unweighting is a random process, which unavoidably discards part of the initial information).

It is hoped that in the future VECBOS will be able to subdivide the phase space in a more suitable way, in order to produce narrower weight distributions. This would allow many studies which at the moment are not possible due to the lack of CPU time as well as of disk space necessary for the production of high statistics samples. 


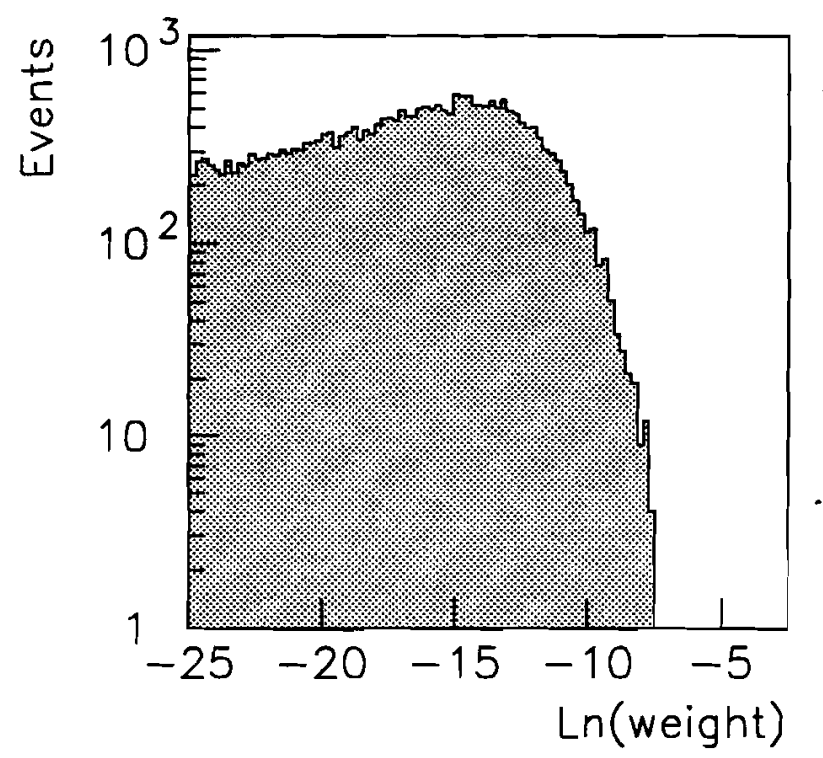

Figure 4.1: Distribution of the logarithm of the weight (ln(weight)) for VECBOS W+3 jet events. The vertical scale is logarithmic.

\subsubsection{Parton fragmentation}

The calculations for $\mathrm{W}+$ jets production are at parton level. These calculations give the transition probabilities between quark and/or gluon states (with some additional particles which interact weakly, such as leptons and electroweak bosons). Quarks and gluons are not the observables of an experiment. However, general QCD theorems guarantee that inclusive quantities calculated using partons should be the same as if calculated after the partons have fragmented, at least to a certain level of approximation. The problem of "translating" a theoretical prediction at parton level to a simulation which gives as output those quantities (jets) which are directly measured in an experiment is not simple. The CDF collaboration developed a simple algorithm (called SETPRT[75]), which transforms partons into jets. This algorithm is based on the independent fragmentation model of Feynman-Field[76] and on a set of phenomenological parameters. The main idea in SETPRT is that there must be a one-to- 
one correspondence between partons and jets. In other words, each parton in the final state must fragment to one single jet. The algorithm based on this principle has been tested on CDF data, tuning the fragmentation parameters of a parton in such a way as to reproduce the observations in an inclusive sample of QCD jets. This approach avoids the ambiguities connected to the possibility that a two-parton final state can produce 3 -jet events. In the meantime it is also a limitation since it does not predict the emission of gluon radiation, which turns out to be part of the underlying event. As a consequence it is not possible to distinguish in the underlying event the contribution coming from the fragmentation of the primary spectator partons (minimum bias) from the radiation coming from the initial and final states of the two partons involved in the large $Q^{2}$ interaction. The underlying event is therefore dependent on the particular process under study.

\subsubsection{Reliability of VECBOS+SETPRT}

Study of a sample of $W+2$ jets

Several checks made in the past by the CDF collaboration in other analyses[41] as well as in this one[77, 78, 79] showed that VECBOS+SETPRT is reliable in describing a wide range of QCD data. As an example one can compare the kinematical distributions of data selected by requiring at least two jets with $\mathrm{E}_{t}>20 \mathrm{GeV}$ and $\mid \eta($ jet $) \mid<2$ (inclusive sample of $\mathrm{W}+2$ jets) with those predicted by VECBOS $W+2$ jets. The $E_{t}\left(\right.$ jet $\left._{1}\right)(a)$ and $E_{t}\left(\right.$ jet $\left._{2}\right)(b)$ distributions for data (solid line) and for VECBOS W+2 jet events (dashed line) are shown in figure 4.2. Montecarlo events were also passed through the fragmentation algorithm SETPRT and through a detector simulation (see section 4.3). The statistical error is shown for VECBOS events. Figures 4.2 (c) and (d) have been obtained requiring two central jets $\left(\mid \cos \theta^{*}\left(\right.\right.$ jet $\left._{1,2}\right) \mid<0.7$, where $\theta^{*}($ jet$)$ is the angle between the jet and the proton beam in the event rest frame, defined without taking into account the longitudinal component of the neutrino momentum). The VECBOS statistical errors have not been shown this time, since they are small for events with central jets. It must be pointed out that in figures 4.2 (c) and (d) one expects about 10 top events for a mass $M_{t o p}=160 \mathrm{GeV} / \mathrm{c}^{2}$. If one compares explicitly the high $\mathrm{E}_{t}$ tail of the distributions 
$\left(\mathrm{E}_{t}\left(\right.\right.$ jet $\left._{1}\right)>100 \mathrm{GeV}, \mathrm{E}_{t}\left(\right.$ jet $\left.\left._{2}\right)>80 \mathrm{GeV}\right)$ for data and Montecarlo, they agree within a standard deviation. More recently a new algorithm called HERPRT[80] has been developed. This algorithm can simulate the parton fragmentation process. HERPRT is an interface between the parton configuration given by VECBOS and HERWIG, a Montecarlo which transforms partons into jets by means of radiation "showers", using QCD algorithms which account for the color coherence[81]. Preliminary studies showed that in the kinematical regions of interest for this analysis, using one or the other fragmentation algorithm doesn't change substantially the conclusions of our study. However, the statistics available at the moment for Montecarlo events with HERPRT are not enough to allow an accurate comparison.

\subsection{ISAJET}

ISAJET[82] is a Montecarlo used by CDF since several years, to simulate the production of $t \bar{t}$ pairs at the Tevatron energy. Also ISAJET, like HERWIG, is a shower Montecarlo. It does not contain the exact matrix element for the gluon radiation. Rather, it is based on perturbative QCD calculations and on phenomenological models for the parton fragmentation. The process of generation can be divided in four stages:

- A "hard" primary scattering is simulated, with the proper cross section as expected from QCD.

- The QCD radiative corrections in the leading log (LO) approximation are added to the initial and final states. These corrections allow the presence of additional partons.

- The partons are fragmented to hadrons, using the independent model of Field and Feynman.

- Jets emitted by the spectator partons are added. Their interaction is simulated as a minimum bias process, to an energy equal to the residual energy. 

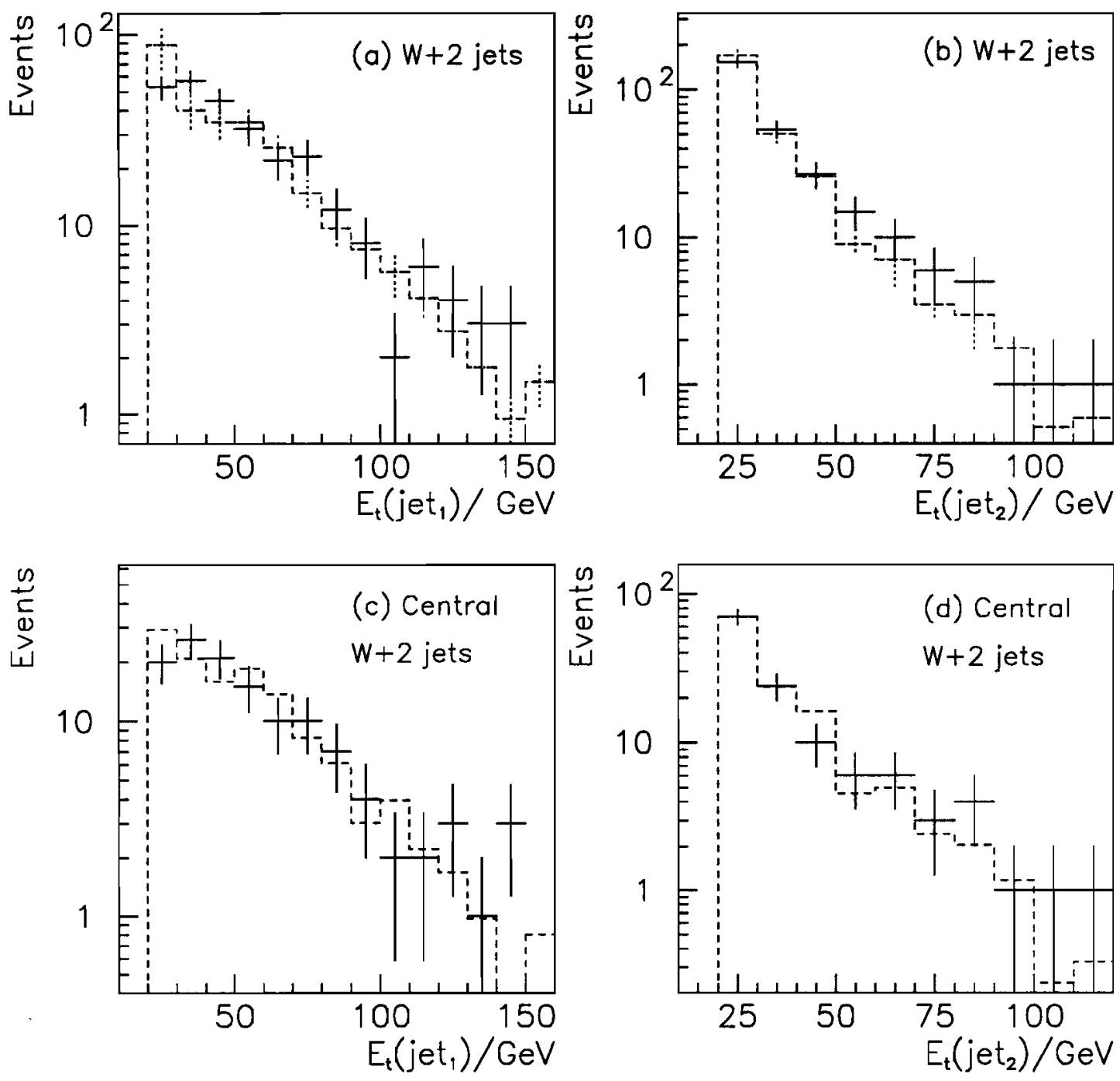

Figure 4.2: Distributions of $E_{t}($ jet $)$ (a) and $E_{t}$ (jet $t_{2}$ (b) for data (points with error bars) and $V E C B O S W+2$ jet events (dashed line). VECBOS has been normalized to the data. Figures (c) and (d) show the corresponding distributions if we require the two jets to be "central" (see the text). 


\subsection{Detector Simulation}

In order to simulate the behavior of Montecarlo generated particles in the detector an official CDF program, called QFL[83], has been used. QFL simulates particles passing through a spherical calorimeter which covers the entire solid angle with the exception of two conical openings in the forward and backward regions. This calorimeter has an electromagnetic and a hadronic part, and is segmented into towers like the CDF calorimeter. The radiation and interaction lengths, as well as the thickness in radiation lengths for the electromagnetic calorimeter and in interaction lengths for the hadronic calorimeter, have been modelled on the basis of the CDF characteristics. Inside the calorimeter a decay region is simulated, contained in a cylindrical volume in which there is a solenoidal magnetic field. The length and radius of the cylinder, as well as the strength of the field, are those of CDF.

QFL is not the most sophisticated detector simulation available at present, but it is the fastest. Since one needs to have high statistics Montecarlo samples to develop the analysis described in this thesis, this solution has been chosen. However, it must be pointed out that our study is essentially based on the information provided by the calorimeter. In this context QFL has been accurately studied and gives results which are highly reliable. 
$-$ 


\section{Chapter 5}

\section{Search for Top in the CDF Data}

In this chapter we select an event sample enriched in top candidates (signal sample, "SS") and a control sample enriched with QCD W events (control sample, "CS"). This selection is obtained using the criteria discussed in subsection 1.4. We then compare some variables which one can use to distinguish signal from background events. As a result a class of events in the signal sample is found which cannot be explained by known QCD processes.

\subsection{Selection of $W+3$ Jet Events}

The selection is based on a sample of inclusive $\mathrm{W}$ events, selected following the criteria described in Chapter 3. In this thesis one wants to concentrate on the investigation of events with one high $\mathrm{P}_{t}$, isolated lepton. Therefore events with a second high $\mathrm{P}_{t}$ isolated lepton candidate track are removed (see section 3.7).

Three jets with corrected $E_{t}(j e t)>20 \mathrm{GeV}$ are then required. For jets with $E_{t} \geq 20 \mathrm{GeV}$, systematic errors in the absolute energy scale and from fluctuations in the calorimeter response have been studied in detail, and they are found to be not too large. A fourth jet is not required, but allowed.

If a small jet reconstruction cone is used (see section 3.3), the jet finding algorithm can sometimes find two jets which are very close to each other. In these cases it is difficult to say whether one single jet is wrongly reconstructed as two jets (either because of fluctuations in the jet fragmentation process, or reconstruction problems in the calorimeter), or whether there 
are really two jets. To avoid ambiguities, the three leading jets are required to be separated from each other in $\mathrm{R}$ by at least 0.7 .

\subsubsection{Signal enriched sample}

From this $W+3$ jet sample a subsample enriched with top candidates is selected, by requiring that the three leading jets are central (see section 1.4). This requirement rejects most of QCD W events, while a large fraction of the top events passes. The angle $\theta^{*}$ (jet) is defined as the angle between the jet and proton beam directions in the center of mass system of the event. The event consists of the $\mathrm{W}$ and all the jets with $E_{t}(j e t)>15 \mathrm{GeV}$. The longitudinal momentum component of the neutrino is ignored. The jets are required to be central by the cuts $\cos \left|\theta^{*}(\mathrm{jet})\right|<0.7$. Table 5.1 summarizes all the cuts which are applied to obtain this event sample of $W+($ at least) 3 jet events enriched with top. We will refer to this event sample in the following as "signal enriched sample" (or briefly "signal sample","SS"). It consists of 15 events (last line of table 5.1), 9 electron and 6 muon events.

In table 5.2 we summarize the number of top events passing the various cuts, based on the ISAJET Montecarlo, for different top masses. A comparison of tables 5.1 and 5.2 shows that the cut on $E_{t}\left(\right.$ jet $\left._{3}\right)$ reduces the number of data events by about a factor of 5.5 , while according to ISAJET top events get reduced only by a factor of 1.2. For a top mass in the range of about $140 \mathrm{GeV} / \mathrm{c}^{2}$ to $180 \mathrm{GeV} / \mathrm{c}^{2}$, one expects between 8 and 2 top events. However, the uncertainty on these numbers coming from the cross section alone is already $30 \%$ about. There are other systematic uncertainties, for example, the way ISAJET handles gluon radiation, and they are difficult to quantify. However, the absolute number of expected events is determined only as a consistency check. To arrive at quantitative results only the shape of kinematical distributions will be used. Seven (eleven) of the 15 events have a fourth jet with $\mathrm{E}_{t}\left(\right.$ jet $\left._{4}\right)>15$ (10) GeV. One event has been removed from the signal sample by di-lepton rejection, as will be discussed in more detail in the next chapter.

One can conclude that, if top exists in the mass range between 140 and $180 \mathrm{GeV} / \mathrm{c}^{2}$, a sample (SS) has been selected where the signal/background ratio is of the order $O(1)$. 


\begin{tabular}{|l|r|}
\hline Cuts applied & Events \\
\hline \hline W Selection $_{1}$ & 14726 \\
$\mathrm{E}_{t}\left(\right.$ jet $\left._{1}\right)>20 \mathrm{GeV}$ & 2097 \\
$\mathrm{E}_{t}\left(\right.$ jet $\left._{2}\right)>20 \mathrm{GeV}$ & 383 \\
$\mathrm{E}_{t}\left(\right.$ jet $\left._{3}\right)>20 \mathrm{GeV}$ & 78 \\
$\Delta \mathrm{R}_{j j}>0.7, \mid \eta\left(\right.$ jet $\left._{1,2,3}\right) \mid<2$. & 46 \\
$\mid \cos \theta^{*}\left(\right.$ jet $\left._{1,2,3}\right) \mid<0.7$ & 15 \\
\hline
\end{tabular}

Table 5.1: Number of data events which pass the various analysis cuts.

\begin{tabular}{|l|lllll|}
\hline$\overline{\mathrm{M}}_{\text {top }}\left(\mathrm{GeV} / \mathrm{c}^{2}\right)$ & 140 & 160 & 170 & 180 & 190 \\
$\sigma(\mathrm{pb})$ & 16.9 & 8.16 & 5.83 & 4.21 & 3.06 \\
\hline Cuts applied & & & & & \\
\hline \hline W selection & $23.3 \pm 1.2$ & $12.3 \pm 0.6$ & $8.7 \pm 0.4$ & $6.6 \pm 0.3$ & $4.7 \pm 0.2$ \\
$\mathrm{E}_{t}\left(\right.$ jet $\left._{1}\right)>20 \mathrm{GeV}$ & $23.3 \pm 1.2$ & $12.3 \pm 0.6$ & $8.7 \pm 0.4$ & $6.6 \pm 0.3$ & $4.7 \pm 0.2$ \\
$\mathrm{E}_{t}\left(\right.$ jet $\left._{2}\right)>20 \mathrm{GeV}$ & $22.5 \pm 1.2$ & $12 . \pm 0.6$ & $8.5 \pm 0.4$ & $6.5 \pm 0.3$ & $4.6 \pm 0.2$ \\
$\mathrm{E}_{t}\left(\right.$ jet $\left._{3}\right)>20 \mathrm{GeV}$ & $18.3 \pm 1$. & $10.5 \pm 0.5$ & $7.6 \pm 0.4$ & $5.8 \pm 0.3$ & $4.2 \pm 0.2$ \\
$\Delta \mathrm{R}_{j j}>0.7, \mid \eta\left(\right.$ jet $\left._{1,2,3}\right) \mid<2$. & $15.5 \pm 1$. & $8.8 \pm 0.5$ & $6.7 \pm 0.4$ & $5.4 \pm 0.3$ & $3.6 \pm 0.2$ \\
$\mid \cos \theta^{*}\left(\right.$ jet $\left._{1,2,3}\right) \mid<0.7$ & $7.6 \pm 0.7$ & $4.5 \pm 0.4$ & $3.2 \pm 0.3$ & $2.4 \pm 0.2$ & $1.8 \pm 0.1$ \\
\hline
\end{tabular}

Table 5.2: Number of top events which pass the various analysis cuts, according to ISAJET, for different top masses. The top production cross section used is from NNLO calculations. We also quote the statistical error.

\subsubsection{Control sample}

The events which have at least one of the three leading jets at $\left|\cos \theta^{*}\right|>0.7$ constitute the control sample CS, enriched with QCD W's. The difference between the last line and the line before in tables 5.1 and 5.2 gives the number of events in the control sample. There are 31 data events in the control sample. By definition, there are no events in common between the signal sample and the control sample. From table 5.2 we see that the number of top events in the signal and the control sample is approximately the same, according to ISAJET. From the control sample, 6 di-lepton candidates have been removed; they will be discussed in the next chapter. 


\subsection{Background}

In the absence of top events we expect the signal and the control sample to be dominated by QCD W+jet events. Contributions from other processes should be relatively small. A systematic study of all the relevant contributions expected for the background has been made.

\subsubsection{W+jets from QCD}

The QCD W+jet production process has been discussed in detail in Chapter 1, where it has been found that the cross section predicted by VECBOS has an uncertainty of about a factor of 2[84] due to the absence of higher order corrections in the matrix element. This uncertainty needs to be taken into account when one interpretes the following numbers. The VECBOS $\mathrm{W}+3$ jets Montecarlo with $\mathrm{q}^{2}=\left\langle\mathrm{P}_{t}\right\rangle^{2}$ predicts about 17 events for the signal sample. For $\mathrm{q}^{2}=\mathrm{M}_{W}^{2}$ about 10 events are predicted. Taking into account the uncertainty of the prediction, these numbers are compatible with both the presence and absence of top events.

\subsubsection{Non-W background}

It has been already discussed that sometimes $\pi$ and $K$ particles can be wrongly identified in the detector as leptons (see Chapter 3). Due to fluctuations in the jet fragmentation process and in the detector response, we can get events which seem to contain a high $\mathrm{P}_{t}$ isolated lepton and missing energy, but do not. The probability that this happens in any given event is very low, but the jet production cross section is much larger than the $\mathrm{W}$ production cross section ${ }^{1}$. In addition, there will be electrons from non-identified photon conversions and leptons from beauty decays which are wrongly taken as coming from a $\mathrm{W}$ decay. One refers to all of these processes as non-W QCD background. Previous studies indicate that this background contributes about $10 \%$ of the $W+$ jets sample[41]. One way to determine the non-W background, commonly applied by several CDF analyses, is to study lepton isolation. Leptons from $\mathrm{W}$ decays are more isolated than lepton candidates from non-W background. In

\footnotetext{
${ }^{1}$ The probability to produce a quark or a gluon with $20<\mathrm{E}_{t}<60 \mathrm{GeV}$ is about 4 to 5 orders of magnitude higher than to produce a lepton by a W decay[85].
} 
this chapter, for simplicity, events which pass the cuts in $\boldsymbol{E}_{t}>25 \mathrm{GeV}$ and $\mathrm{M}_{\text {trans }}>40 \mathrm{GeV} / \mathrm{c}^{2}$ will be called "W events", and those which do not pass these cuts will be called "non-W events". When one loses the lepton isolation cut (Iso) one gets more events, as shown in tables 5.3 and 5.4. For Iso $<0.1$ there are 19 (39) non-W events in the signal (control) sample and 15 (31) W events.

\begin{tabular}{|l|rr|}
\hline \multicolumn{3}{|c|}{ Signal Sample } \\
\hline \hline & non-W & W \\
\hline \hline Iso $<0.1$ & 19 & 15 \\
\hline $0.1<$ Iso $<0.5$ & 33 & 1 \\
\hline
\end{tabular}

Table 5.3: $W$ and non- $W$ events in the signal sample for different isolation cuts.

\begin{tabular}{|l|rr|}
\hline \multicolumn{3}{|c|}{ Control Sample } \\
\hline \hline & non-W & W \\
\hline \hline Iso $<0.1$ & 39 & 31 \\
\hline $0.1<$ Iso $<0.5$ & 86 & 5 \\
\hline
\end{tabular}

Table 5.4: $W$ and non- $W$ events in the control sample for different isolation cuts.

For $0.1<$ Iso $<0.5$ the non-W background in the signal (control) sample increases by a factor of $\frac{33}{19}=1.74\left(\frac{86}{39}=2.2\right)$. Within this isolation range, there is one $W$ event in the signal sample and there are $5 \mathrm{~W}$ events in the control sample, so that one can conclude that in the SS and CS samples the expected non-W background is $\frac{1}{1.74}=0.6 \pm 0.6$ events which is $(4 \pm 4) \%$, and $\frac{5}{2.2}=2.3 \pm 1$. events which is $(7 \pm 3) \%^{2}$.

\subsubsection{Di-bosons}

$\mathrm{W}^{+} \mathrm{W}^{-}$events are a physics background for a top search which uses the event structure. If one of the W's decays leptonically and the other one hadronically, then the presence of additional jets from gluon radiation can create the signature $l \nu_{l} j j j$. The largest contribution to the $\mathrm{W}^{+} \mathrm{W}^{-}$production comes from $q \bar{q}$ annihilation. The second largest comes from gluon fusion (about $20-25 \%$ of the $q \bar{q}$ annihilation), then $\mathrm{W}^{+} \mathrm{W}^{-}$fusion (one order of magnitude

\footnotetext{
${ }^{2}$ We are assuming here that $\boldsymbol{F}_{t}$ and isolation are not correlated. This assumption always has been used in the CDF analysis[85] and no contrary evidence has been found.
} 
smaller) and ZZ fusion. To simulate these events the ISAJET Montecarlo has been used. The $\mathrm{W}^{+} \mathrm{W}^{-}$production cross section has been obtained with a NLO calculation[86]. At parton level this means that one takes into account contributions from the processes $2 \rightarrow 3$ $\left(q \bar{q} \rightarrow W^{+} W^{-} g, q g \rightarrow W^{+} W^{-} q, q \bar{g} \rightarrow W^{+} W^{-} g\right.$ ), and from one-loop corrections to the $2 \rightarrow 2$ process $q \bar{q} \rightarrow W^{+} W^{-} .{ }^{3}$ In table 5.5 we summarize the number of events which pass the various cuts. The error on the cross section is about $30 \%$. From table 5.5 one expects about $1 \mathrm{WW}$

\begin{tabular}{|l|c|}
\hline Analysis cuts & Events $(\sigma=9.5 \mathrm{pb})$ \\
\hline \hline $\mathrm{W}$ selection & $13.3 \pm 0.8$ \\
$\mathrm{E}_{t}\left(\right.$ jet $\left._{1}\right)>20 \mathrm{GeV}$ & $12 . \pm 0.8$ \\
$\mathrm{E}_{t}\left(\right.$ jet $\left._{2}\right)>20 \mathrm{GeV}$ & $7.7 . \pm 0.6$ \\
$\mathrm{E}_{t}\left(\right.$ jet $\left._{3}\right)>20 \mathrm{GeV}$ & $3.5 \pm 0.4$ \\
$\Delta \mathrm{R}_{j j}>0.7, \mid \eta\left(\right.$ jet $\left._{1,2,3}\right) \mid<2$. & $2.6 \pm 0.4$ \\
$\mid \cos ^{*}\left(\right.$ jet $\left._{1,2,3}\right) \mid<0.7$ & $1 . \pm 0.2$ \\
\hline
\end{tabular}

Table 5.5: Number of WW events passing the various analysis cuts, according to ISAJET and based on the NLO cross section. Statistical errors are also shown.

event in the signal sample, and 1.6 events in the control sample (difference between last line and line before).

Also WZ production contributes to the physics background when the $\mathrm{W}$ decays leptonically and the $\mathrm{Z}$ hadronically. Again ISAJET is used to simulate the events and the cross section is a NLO calculation[86]. In the signal sample 0.1 events are expected and in the control sample 0.16 events are expected (compare table 5.6).

\begin{tabular}{|l|c|}
\hline Analysis cuts & Events $(\sigma=2.6 \mathrm{pb})$ \\
\hline \hline W selection & $2.2 \pm 0.2$ \\
$\mathrm{E}_{t}\left(\right.$ jet $\left._{1}\right)>20 \mathrm{GeV}$ & $1.9 \pm 0.2$ \\
$\mathrm{E}_{t}\left(\right.$ jet $\left._{2}\right)>20 \mathrm{GeV}$ & $1.5 . \pm 0.2$ \\
$\mathrm{E}_{t}\left(\right.$ jet $\left._{3}\right)>20 \mathrm{GeV}$ & $0.48 \pm 0.08$ \\
$\Delta \mathrm{R}_{j j}>0.7, \mid \eta\left(\right.$ jet $\left._{1,2,3}\right) \mid<2$. & $0.26 \pm 0.06$ \\
$\mid \cos \theta^{*}\left(\right.$ jet $\left._{1,2,3}\right) \mid<0.7$ & $0.1 \pm 0.04$ \\
\hline
\end{tabular}

Table 5.6: WZ events passing the various analysis cuts, according to ISAJET, based on the $N L O$ cross section. Statistical errors are also shown.

\footnotetext{
${ }^{3}$ This calculation is restricted to corrections of order $\alpha$ and so the contribution from gluon fusion, which is of order $\alpha^{2}$, is not taken into account.
} 


\subsection{Choice of Kinematical Variables}

Once the signal sample is selected, one needs to find out whether there is a non-standard QCD component. The absolute predictions from the VECBOS Montecarlo have an uncertainty of about a factor of 2 . Therefore we are comparing the kinematical properties of the events to the predictions from VECBOS and ISAJET, and not the absolute numbers.

We begin with a study of various simple kinematical variables (or combinations of variables) to find out how to best separate signal from background.

Montecarlo studies show that after applying the analysis cuts, the $\mathrm{E}_{t}$ (jet) spectra of $\mathrm{QCD}$ W events and heavy top decays are much different. Figures 5.1 (a), (b) and (c) show the distributions of $E_{t}\left(\right.$ jet $\left._{1}\right), E_{t}\left(\right.$ jet $\left._{2}\right)$ and $E_{t}\left(\right.$ jet $\left._{3}\right)$ for top events from ISAJET $\left(M_{\text {top }}=170 \mathrm{GeV} / \mathrm{c}^{2}\right)$ $\left(f_{i}^{t 170}\left(x_{i}\right)\right.$, with $\left.i=a, b, c\right)$ and VECBOS $\mathbf{W}+3$ jet events $\left(f_{i}^{Q C D}\left(x_{i}\right)\right.$, with $\left.i=a, b, c\right)$, after all analysis cuts. To find out which of the $x_{i}$ variables can give the best separation signalbackground, the functions

$$
F_{i}(x)=\int_{0}^{\bar{x}_{i}} f_{i}\left(x_{i}\right) d x_{i}
$$

are used. These functions are the integral of $f_{i}\left(x_{i}\right)$ from 0 and some value $\bar{x}_{i}$ of the variable $x_{i}$. Figure 5.2 (a) compares the separation power of the three variables $E_{t}\left(\right.$ jet $\left._{1}\right), E_{t}\left(\right.$ jet $\left._{2}\right)$ and $\mathrm{E}_{t}\left(\right.$ jet $\left._{3}\right)$. The $\mathrm{x}$ axis shows the value of $F(x)$ for top $\left(\mathrm{M}_{\text {top }}=170 \mathrm{GeV} / \mathrm{c}^{2}\right)$. The figure shows that $\mathrm{E}_{t}\left(\right.$ jet $\left._{1}\right)$ has the smallest separation power while $\mathrm{E}_{t}\left(\right.$ jet $\left._{3}\right)$ is the most powerful among these three variables. In order to see if the events are more compatible with background or with a top signal, one can also use a relative likelihood: $\mathrm{rL}$, defined using the $\mathrm{E}_{t}\left(\mathrm{jet}_{2}\right)$ and $\mathrm{E}_{t}\left(\mathrm{jet}_{3}\right)$ variables. As a first step, referring to the functions $f_{i}\left(x_{i}\right)(i=a, b, c)$ which have been shown in figure $5.1(\mathrm{a}),(\mathrm{b}),(\mathrm{c})$, the product aL (absolute likelihood) is defined as:

$$
a L=\prod_{i=b}^{c} f_{i}\left(\bar{x}_{i}\right)
$$

where the $f_{i}$ refer to a certain expectation (QCD or top). rL for an event characterized by a certain value $\bar{x}_{i}$ for each of the variables, will be therefore defined as:

$$
r L=\prod_{i=b}^{c} \frac{f_{i}^{t 170}\left(\bar{x}_{i}\right)}{f_{i}^{Q C D}\left(\bar{x}_{i}\right)}=\frac{a L^{t 170}}{a L^{Q C D}}
$$



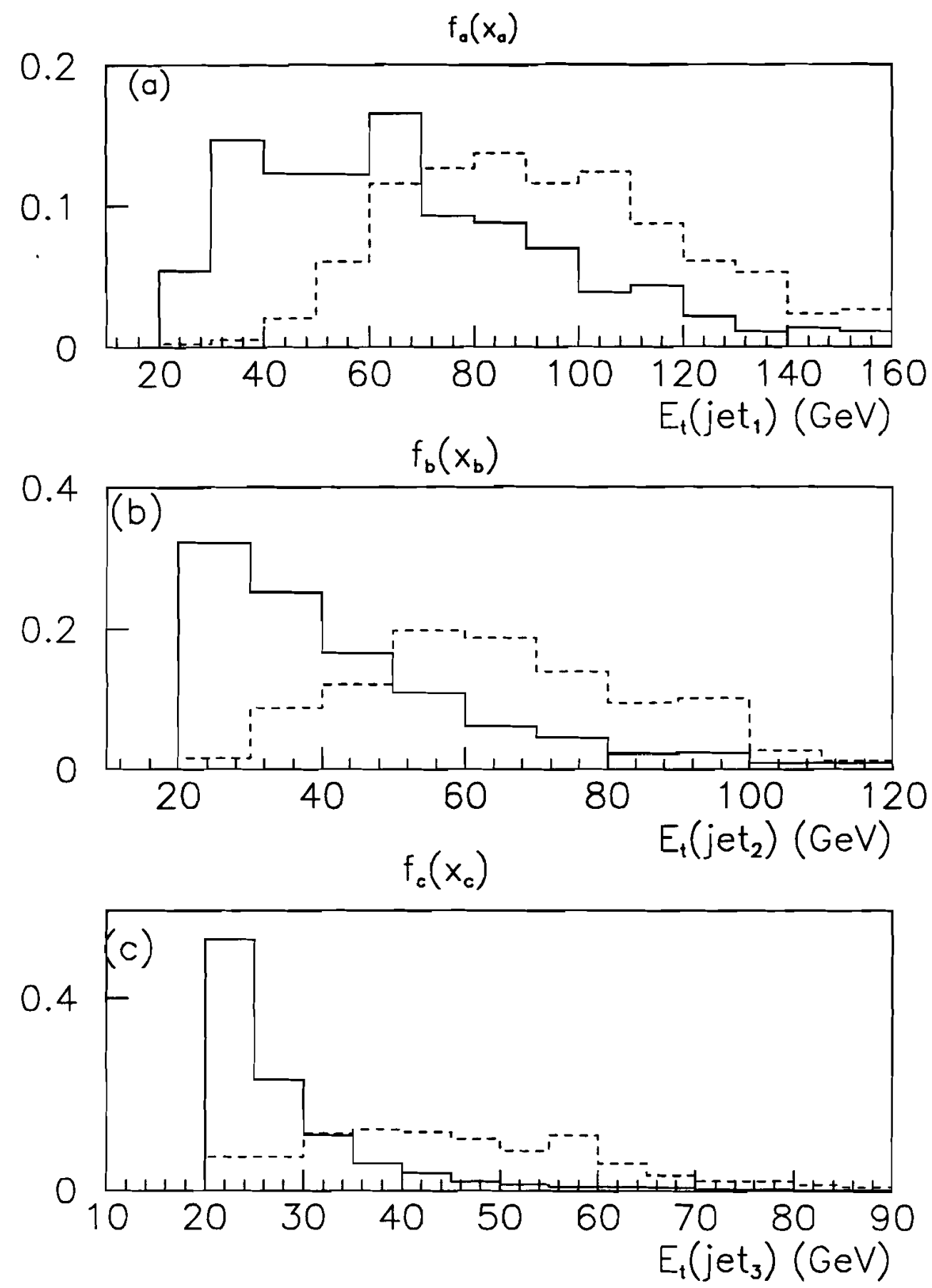

Figure 5.1: Distribution in $E_{t}\left(\right.$ jet $\left._{1}\right)(a), E_{t}\left(\right.$ jet $\left._{2}\right)(b)$ and $E_{t}\left(\right.$ jet $\left._{3}\right)$ (c) for top events $\left(M_{\text {top }}=170\right.$ $\left.\mathrm{GeV} / \mathrm{c}^{2}\right)$ (dotted line) and VECBOS $W+3$ jet (solid line). 


\section{Signal-background separation}
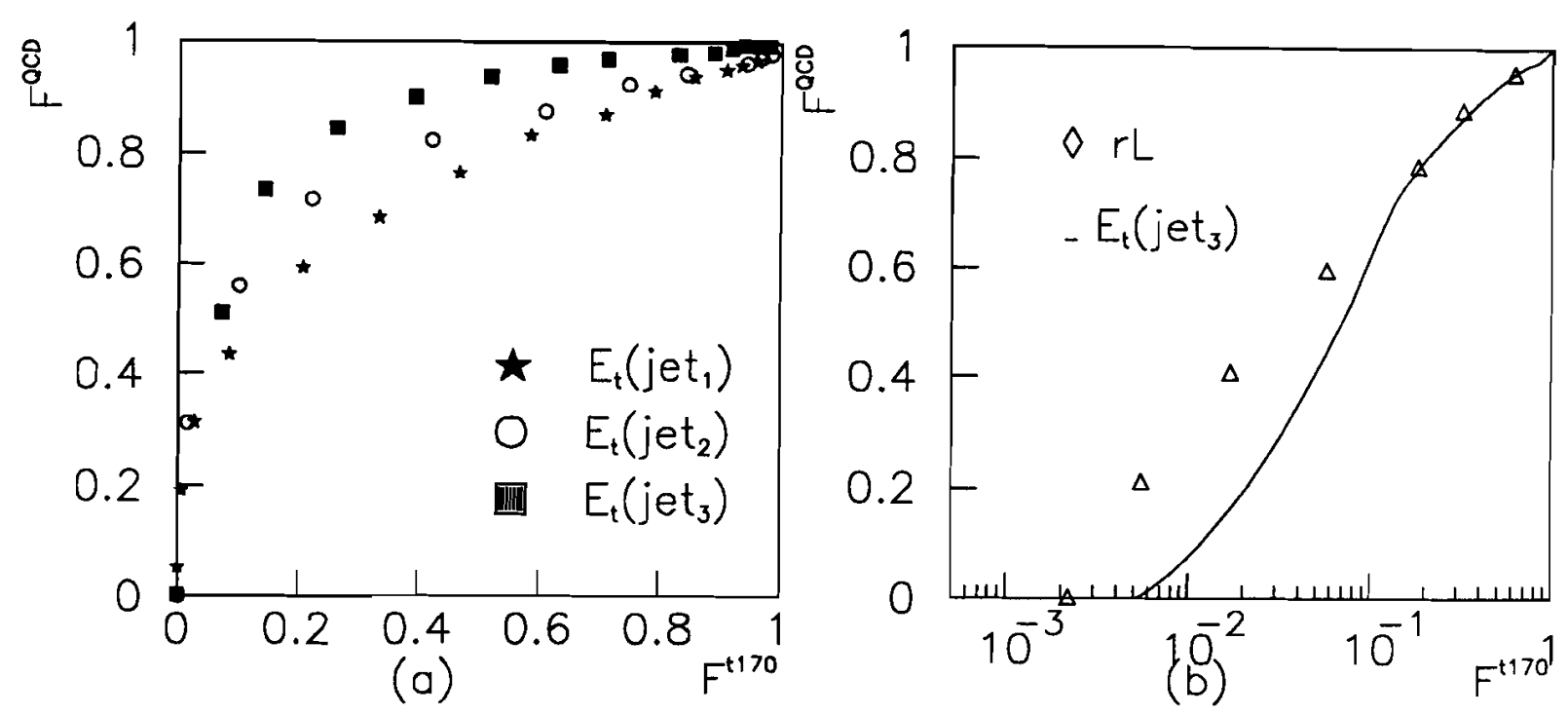

Figure 5.2: (a) Signal-background separation power of the three variables $E_{t}\left(\right.$ jet $\left._{1}\right), E_{t}\left(\right.$ jet $\left._{2}\right)$ and $E_{t}\left(j e t_{3}\right)$; (b) Comparison between $E_{t}\left(j e t_{3}\right)$ and $R L$ based on $E_{t}\left(j e t_{2}\right)$ and $E_{t}\left(j e t_{3}\right)$. The $x$ axis has a logarithmic scale.

Clearly $\mathrm{rL}$ is not a probability, since the variables used (the jet $\mathrm{E}_{t}$ 's) are correlated. The variables $\mathrm{E}_{t}\left(\right.$ jet $\left._{3}\right)$ and $\mathrm{rL}$, defined as in equation 5.3.3, are compared in figure 5.2 (b). One observes that $\mathrm{rL}$ has a slightly better discrimination power with respect to $\mathrm{E}_{t}\left(\mathrm{jet}_{3}\right)$. The $\operatorname{differ}$ ence between the two variables increases as one get closer to the left lower region of figure 5.2. This region corresponds to $f_{i}$ values (see equation 5.3.1) calculated at low $\mathrm{E}_{t}$, where the background dominates on the top signal. Finally, $\mathrm{rL}$ and the sum $\mathrm{E}_{t}\left(\mathrm{jet}_{2}\right)+\mathrm{E}_{t}\left(\mathrm{jet}_{3}\right)($ figure $\mathrm{s.3}(\mathrm{a}))$ have been compared, as well as the variables $E_{t}\left(\right.$ jet $\left._{2}\right)+E_{t}\left(\right.$ jet $\left._{3}\right)$ and $E_{t}\left(\right.$ jet $\left._{2}\right)+E_{t}\left(\right.$ jet $\left._{3}\right)+E_{t}\left(\right.$ jet $\left._{1}\right)$ (figure $5.3(\mathrm{~b})$ ). It must be pointed out that adding $\mathrm{E}_{t}\left(\mathrm{jet}_{1}\right)$ does not add more information. $\mathrm{rL}$ is therefore the variable which is most sensitive to the differences between signal and background. On the other hand, it is reasonable to expect that by using multidimensional distributions of kinematical variables (as an example $\mathrm{E}_{t}\left(\right.$ jet $\left._{2}\right)$ vs. $\mathrm{E}_{t}\left(\mathrm{jet}_{3}\right)$ ) as well as a rL defined by more variables, a better discrimination power can be obtained. The variables which 


\section{Signal-background separation}
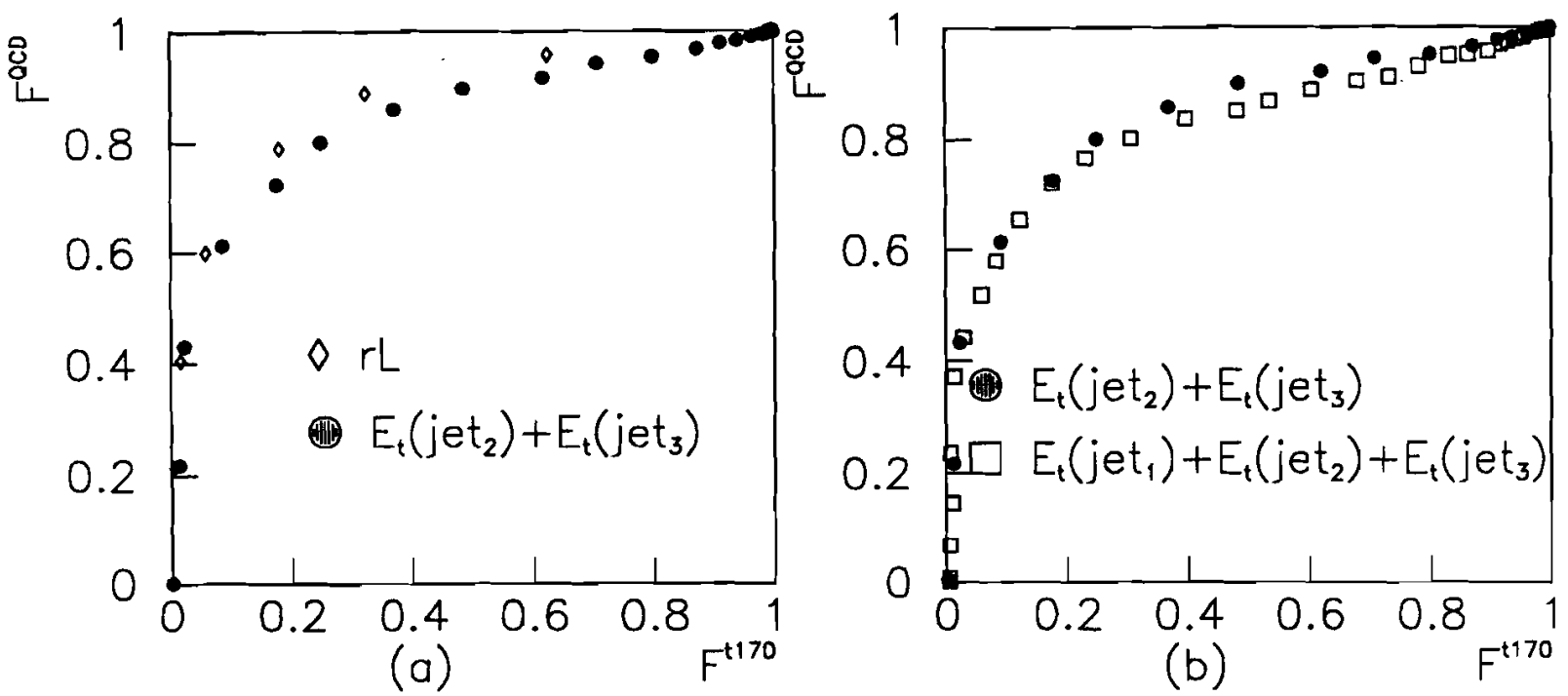

Figure 5.3: Discrimination power for background and signal between the variables $r L$ and $E_{t}\left(\right.$ jet $\left._{2}\right)+E_{t}\left(j e t_{3}\right)(a)$, and between $E_{t}\left(\right.$ jet $\left._{2}\right)+E_{t}\left(j e t_{3}\right)$ and $E_{t}\left(j e t_{1}\right)+E_{t}\left(j e t_{2}\right)+E_{t}\left(j e t_{3}\right)(b)$.

have been checked are a compromise between the desire to obtain a clear background-signal separation, which can help in the quantitative interpretation of the selected events, and the will to arrive at a very simple choice. Therefore the $\mathrm{rL}$ based on $\mathrm{E}_{t}\left(\right.$ jet $\left._{2}\right)$ and $\mathrm{E}_{t}\left(\right.$ jet $\left._{3}\right)$ has been used to quantify the observations on the selected data samples.

\subsection{Identification of Top Candidates}

In table 5.7 the jet transverse energy and the lepton momentum of the 15 events of the SS sample are listed. From table 5.7 one sees that many of these events have very energetic jets. The $E_{t}\left(\right.$ jet $\left._{1}\right)(a), E_{t}\left(\right.$ jet $\left._{2}\right)(b)$ ed $E_{t}\left(\right.$ jet $\left._{3}\right)(c)$ distributions for the 15 events are shown in figure 5.4. The data distributions look harder and more similar to the distributions for a heavy top (in figure 5.1, dashed line), compared to the correspondig spectra for the VECBOS events (in figure 5.1 , solid line). In order to quantify these observations, we use the rL 

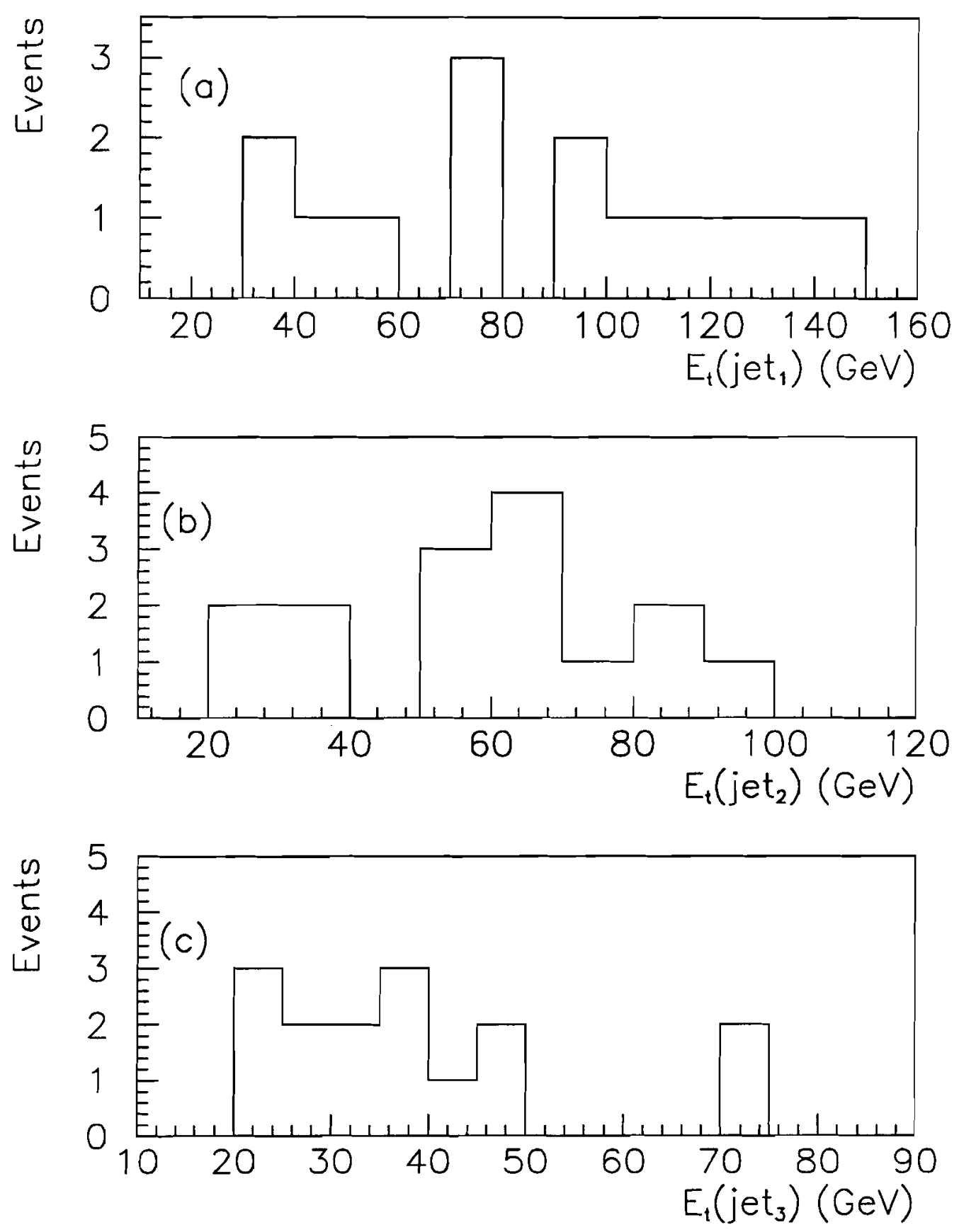

Figure 5.4: $E_{t}\left(\right.$ jet $\left._{1}\right)$ (a), $E_{t}\left(\right.$ jet $\left._{2}\right)$ (b) and $E_{t}\left(\right.$ jet $\left.t_{3}\right)$ (c) distributions for the 15 events. In the $E_{t}\left(j e t_{1}\right)$ spectrum one of the event has $E_{t}=223 \mathrm{GeV}$, and therefore is outside the histogram. 


\begin{tabular}{|l|lllll|}
\hline Run-Event & $\begin{array}{l}\mathrm{E}_{t}\left(\text { jet }_{1}\right) \\
(\mathrm{GeV})\end{array}$ & $\begin{array}{l}\mathrm{E}_{t}\left(\text { jet }_{2}\right) \\
(\mathrm{GeV})\end{array}$ & $\begin{array}{l}\mathrm{E}_{t}\left(\text { jet }_{3}\right) \\
(\mathrm{GeV})\end{array}$ & $\begin{array}{l}\mathrm{E}_{t}\left(\text { jet }_{4}\right) \\
(\mathrm{GeV})\end{array}$ & $\begin{array}{l}\mathrm{P}_{t}(\text { lepton }) \\
(\mathrm{GeV} / \mathrm{c})\end{array}$ \\
\hline \hline $40758-44414$ & 95.1 & 83.4 & 71.4 & 31.4 & 113.1 \\
\hline $42913-59303$ & 127.8 & 82.5 & 38.2 & 9.8 & 30.5 \\
\hline $43096-47223$ & 137.0 & 79.4 & 73.0 & 38.5 & 33.8 \\
\hline $44931-59686$ & 36.9 & 28.6 & 25.0 & 12.2 & 56.4 \\
\hline $45705-54765$ & 96.8 & 51.7 & 47.8 & 26.4 & 49.8 \\
\hline $45779-6523$ & 78.5 & 62.5 & 34.6 & 20.4 & 52.2 \\
\hline $45801-80320$ & 43.5 & 30.3 & 29.3 & 14.4 & 37.1 \\
\hline $45902-240098$ & 71.6 & 52.4 & 23.7 & 15.0 & 57.4 \\
\hline $47616-24577$ & 59.2 & 29.1 & 21.0 & 0.0 & 26.6 \\
\hline \hline $42517-44047$ & 37.3 & 31.8 & 27.7 & 10.8 & 49.7 \\
\hline $42539-200087$ & 109.6 & 67.9 & 41.7 & 36.5 & 44.9 \\
\hline $43336-248916$ & 110.6 & 67.2 & 36.5 & 9.9 & 43.2 \\
\hline $43351-266423$ & 145.3 & 90.7 & 31.5 & 19.4 & 24.1 \\
\hline $43276-101844$ & 223.8 & 50.8 & 37.5 & 9.4 & 179.8 \\
\hline $45753-79414$ & 80.3 & 68.2 & 45.3 & 19.0 & 70. \\
\hline
\end{tabular}

Table 5.7: Parameters of the 15 events of the top enriched sample.

previously defined. In figure 5.5 (a) we show how VECBOS $W+3$ jet events and ISAJET events $\left(M_{t o p}=170 \mathrm{GeV} / \mathrm{c}^{2}\right)$ are distributed in $\ln (\mathrm{rL})$. In VECBOS $\mathrm{q}^{2}=<\mathrm{P}_{t}>^{2}$ has been used as parameter in the $\alpha_{s}\left(q^{2}\right)$ coupling costant. The cross sections are normalized to 1 after having applied the cuts which define the SS. The two distributions are enough separated so that a top signal can be seen, provided that the signal-background ratio in the SS sample be of the order $\mathrm{O}(1)$. In figure 5.5 (b) the data events are shown, together with the expectation for VECBOS $\mathrm{W}+3$ jets (from figure 5.5 (a)). The VECBOS prediction has been normalized to data in the region $\ln (\mathrm{rL})<0$, where there are 5 data events, and is shown with the associated statistical error. The data are not distributed as one would expect from a pure QCD sample. Instead, an excess of events is observed at $\ln (r L)>0$. Figure 5.6 (a) shows the distributions in $\ln (r L)$ as predicted by VECBOS and ISAJET for the CS sample. In figure 5.6 (b) the distribution in $\ln (\mathrm{r} \mathrm{L})$ for the 31 data events which belong to the CS sample is compared with the VECBOS prediction. Again, VECBOS has been normalized to the data observed at $\ln (\mathrm{r} L)<0$ and it is shown with its statistical error. There is good agreement between data and Montecarlo in the region which is expected to be dominated by $\mathrm{QCD}(\ln (\mathrm{rL})<0)$. However, there is an excess 

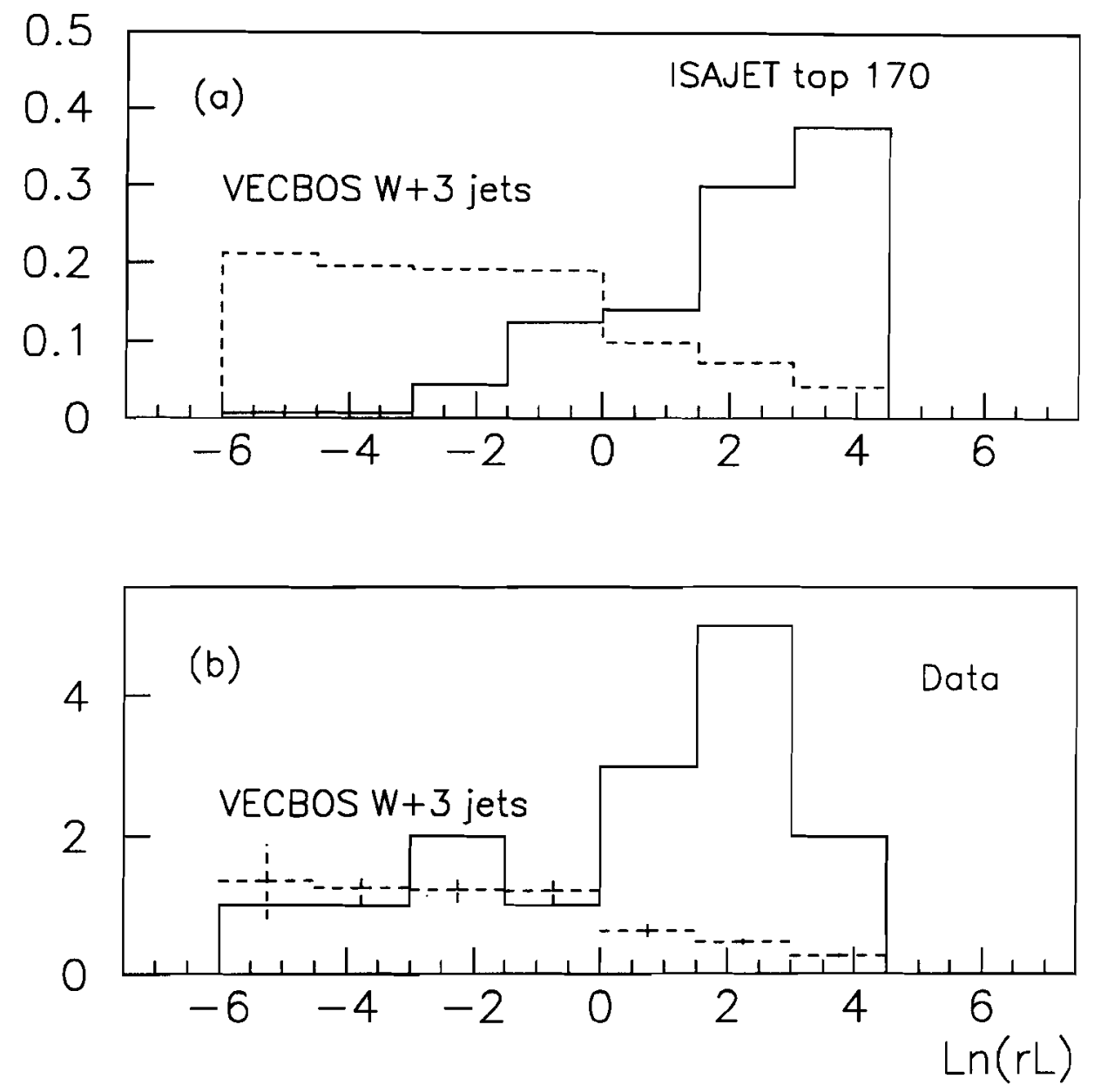

Figure 5.5: (a) Distribution in $\ln (r L)$ for VECBOS $W+3$ jets and ISAJET ( $M_{\text {top }}=170$ $\left.G e V / c^{2}\right)$; (b) the same distribution for data and VECBOS W+3 jets, having normalized Montecarlo events to data in the region $\ln (r L)<0$. 

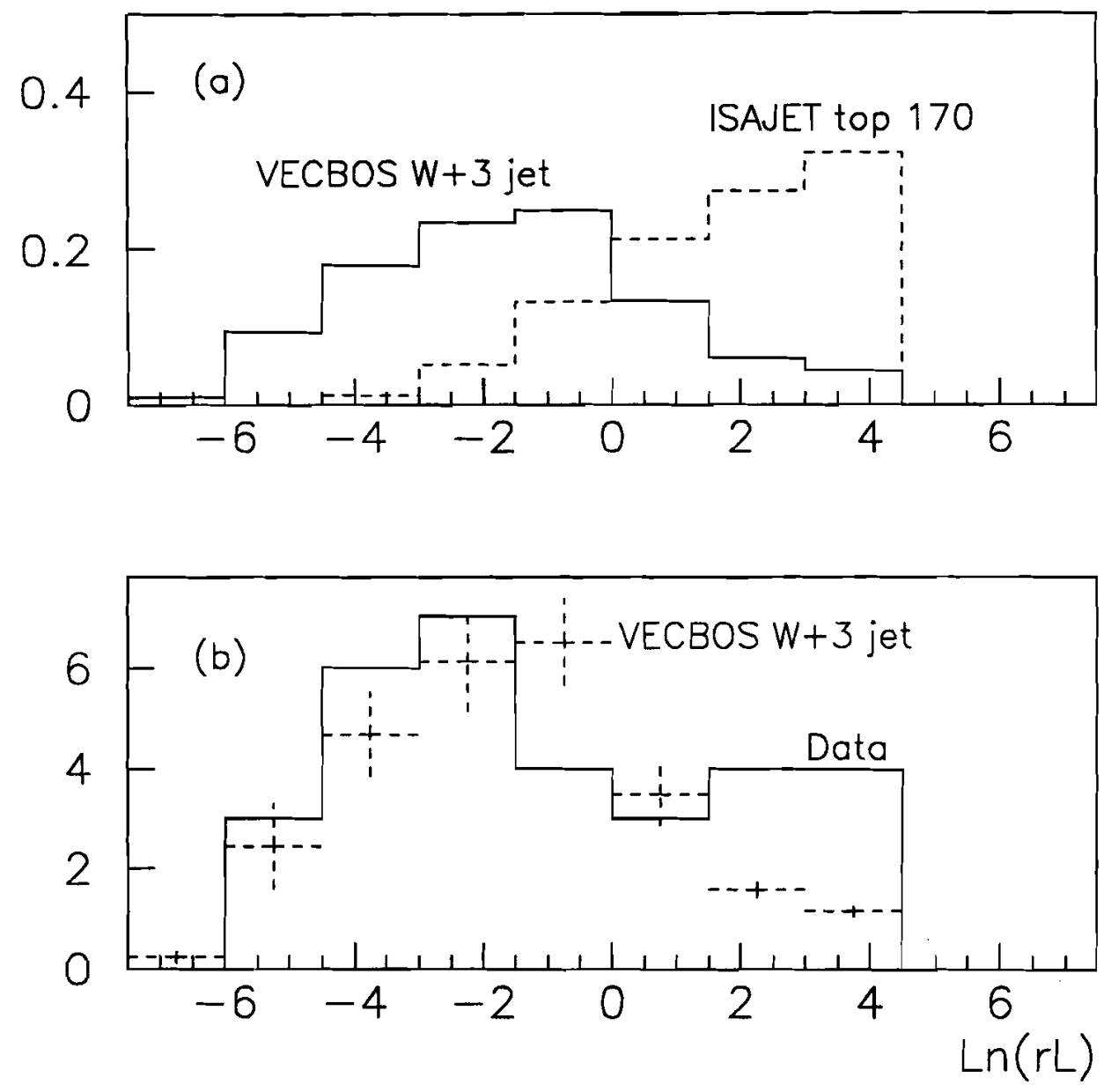

Figure 5.6: (a) Distribution in $\ln (r L)$ for $V E C B O S W+3$ jets and ISAJET (M $M_{\text {top }}=170$ $\left.G e V / c^{2}\right)$. (b) Distribution in $\ln (r L)$ for data and VECBOS $W+3$ jets. The Montecarlo has been normalized to the data in the region $\ln (r L)<0$. 
of data in the region at $\ln (r \mathrm{~L})>0$. ISAJET predicts a number of top events in the CS which is about the same as in the SS sample. Due to the limited statistics available, the observed excess is not inconsistent with this prediction.

\subsection{Statistical and Systematic Errors}

We try to find an explanation for the excess of data in the $S S$ at $\ln (r L)>0$.

\subsubsection{Effects from changing VECBOS parameters on the predic- tions}

In order to estimate the systematic error due to the use of a $W+3$ jets Montecarlo based on the tree level matrix element, the results obtained using two different $q^{2}$ for $\alpha_{s}$ have been compared. The choice of $\mathrm{q}^{2}=\mathrm{M}_{W}{ }^{2}$ should result in harder $\mathrm{E}_{t}\left(\right.$ jet $\left._{2}\right)$ and $\mathrm{E}_{t}\left(\right.$ jet $\left._{3}\right)$ spectra. The result obtained with VECBOS $\mathrm{W}+4$ jets has been also compared. For $\mathrm{E}_{t}\left(\mathrm{jet}_{3}\right) \gg \mathrm{P}_{t}($ parton) cut, the $\mathrm{E}_{t}\left(\right.$ jet $\left._{1,2,3}\right)$ spectra are very similar for VECBOS $\mathrm{W}+3$ and VECBOS $\mathrm{W}+4$ jet events. In table 5.8 the first column shows the fraction of VECBOS events at $\ln (\mathrm{r} L)<0$. In this region VECBOS is normalized to the number of observed data events, that is 5 . The second column shows the number of expected events at $\ln (r L)>0$, based on this normalization. One finds

\begin{tabular}{|l|ccc|}
\hline & Montecarlo & Obser. at & Expect.QCD \\
\hline & at $\ln (\mathrm{RL})<0$ & $\ln (\mathrm{RL})<0$ & $\ln (\mathrm{RL})>0$ \\
\hline \hline $\mathrm{W}+3$ jet $\mathrm{q}^{2}=<P_{\mathrm{t}}>^{2}$ & $79 \%_{-2.7}^{+2.3 \%}$ & 5 & $1.34_{-.53}^{+.75}$ events \\
\hline $\mathrm{W}+3$ jet $\mathrm{q}^{2}=\mathrm{M}_{W}{ }^{2}$ & $72 \%_{-3.7}^{+3.1 \%}$ & 5 & $1.92_{-.77}^{+1.10}$ events \\
\hline $\mathrm{W}+4$ jet & $66 \%_{-5.9}^{+5.5} \%$ & 5 & $2.56_{-1.07}^{+1.62}$ events \\
\hline
\end{tabular}

Table 5.8: Percentage of $Q C D$ events at $\ln (r L)>0$ as predicted by different VECBOS samples. The errors in the first column are the statistical errors from the Montecarlo sample (typically 15\%). The second column shows the predicted number of $Q C D$. events at $\ln (r L)>0$, after assuming that the 5 observed events at $\ln (r L)<0$ are $Q C D$. The errors in the third column include the statistical error on the 5 observed events (50\%).

that at $\ln (\mathrm{rL})>0$ the expectations are in all cases well below the observed 10 events. 


\subsubsection{Uncertainty on the ISAJET Montecarlo predictions}

The likelihood $\mathrm{rL}$ is based on a top mass of $170 \mathrm{GeV} / \mathrm{c}^{2}\left(\mathrm{rL}^{t 170}\right)$.

In order to know which top mass better describes our data in terms of the $\mathrm{rL}$, one needs to compare rL distributions obtained by using different top masses. Determining the top mass will require dedicated studies. For the time being distribution of top events of different masses has been studied, using the same variable $\mathrm{rL}^{t 170}$. In table 5.9 the percentage of events at $\ln \left(\mathrm{rL}^{t 170}\right)<0$ is shown, for several top masses. This percentage increases as the top mass is de-

\begin{tabular}{|c|c|}
\hline $\mathbf{M}_{\text {top }}\left(\mathrm{GeV} / \mathrm{c}^{2}\right)$ & Events at $\ln (\mathrm{rL})<0$ \\
\hline 140 & $34 \% \pm 3 \%$ \\
\hline 160 & $20 \% \pm 3 \%$ \\
\hline 170 & $18 \% \pm 2 \%$ \\
\hline 180 & $12 \% \pm 2 \%$ \\
\hline 190 & $11 \% \pm 2 \%$ \\
\hline 220 & $6 \% \pm 1 \%$ \\
\hline
\end{tabular}

Table 5.9: Fraction of top events at $\ln \left(r L^{170}\right)<0$, for several top masses.

creasing. For $\mathrm{M}_{\text {top }}>160 \mathrm{GeV} / \mathrm{c}^{2}$ at least $80 \%$ of the events are at $\ln (\mathrm{rL})>0$. The requirement $\ln (r \mathrm{~L})>0$ is therefore a good identification of top events, not much dependent on the exact value of the (unknown) top mass.

The $\mathrm{rL}$ answers the question whether a given event is more compatible with QCD background or with top. On the other hand the aL, which has been used to define the $\mathrm{rL}$, allows to explicitely quantify the agreement with the various expectations. The distribution in $\ln \left(\mathrm{aL}^{Q C D}\right)$ of the VECBOS W+3 jet events is shown in figure 5.7 (a). Figure 5.7 (b) shows the distribution in the same variable for the 15 data events which belong to the SS. There are 10 events at $\ln \left(a L^{Q C D}\right)<-4$ where only 1.5 are expected, according to the VECBOS prediction (extrapolating from the region $\left.\ln \left(\mathrm{aL}^{Q C D}\right)>-4\right)$. The distribution in $\ln \left(\mathrm{aL}{ }^{Q C D}\right)$ for top events generated with ISAJET is shown in figure 5.7 (c) for $\mathbf{M}_{\text {top }}=170 \mathrm{GeV} / \mathrm{c}^{2}$ (points with error bars) and for $M_{\text {top }}=180 \mathrm{GeV} / \mathrm{c}^{2}$ (dashed line). It has been shown[87] already that the ISAJET jet activity can depend on how the effect of gluon radiation is handled in the Montecarlo. It is not possible to prove that ISAJET simulates correctly gluon radiation in top events, without 

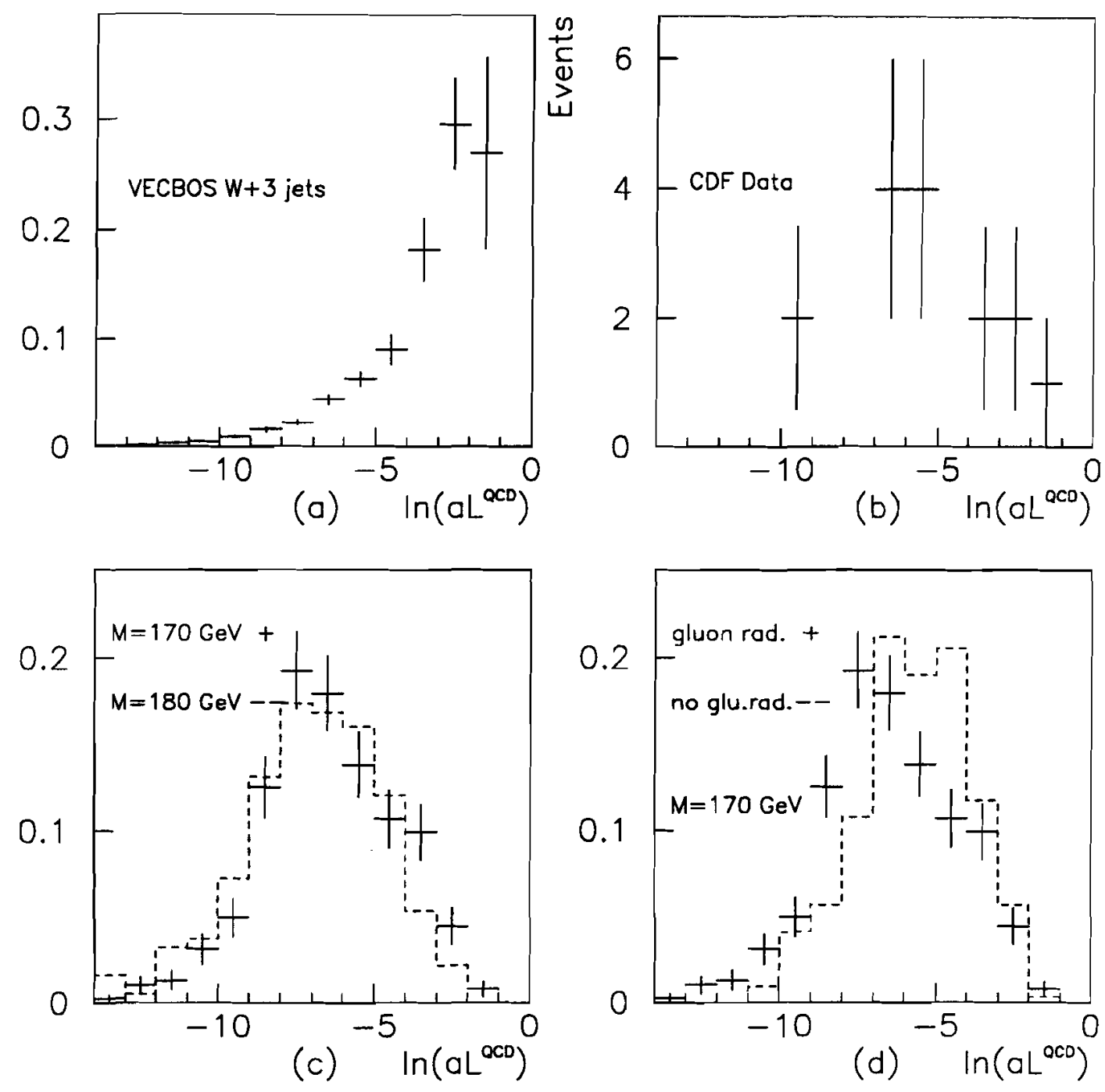

Figure 5.7: Distribution in $\ln \left(a L^{Q C D}\right)$ for (a) VECBOS $W+3$ jet, (b) for the CS data, (c) for Montecarlo top events with $M_{\text {top }}=170 \mathrm{GeV} / \mathrm{c}^{2}$ (points with error bars)) and $M_{\text {top }}=180 \mathrm{GeV} / \mathrm{c}^{2}$ (dashed line), (d) for Montecarlo top events with $M_{t o p}=170 \mathrm{GeV} / \mathrm{c}^{2}$ with gluon radiation activated (points with error bars) and without gluon radiation (dashed line). 
having a large sample of top events to compare. Therefore it is difficult to quantify the corresponding systematic uncertainty. In order to get a rough estimate on how large it might be, the results obtained with and without the option which allows one to activate gluon radiation production in ISAJET have been compared. The results are shown in figure 5.7 (d), where the distribution in $\ln \left(\mathrm{aL}^{Q C D}\right)$ for top events $\left(\mathrm{M}_{t}=170 \mathrm{GeV} / \mathrm{c}^{2}\right)$ has been obtained with (points with error bars) and without 4 (dashed line) gluon radiation. From this comparison one sees that gluon radiation might change the $E_{t}$ jet spectra a lot. Gluon radiation might have as strong an effect on the $E_{t}$ jet spectra as a mass increase of 10 to $20 \mathrm{GeV} / \mathrm{c}^{2}$. Figures 5.7 (b) e (d) show that the distribution in $a L^{Q C D}$ is only weakly sensitive to the top mass, and that a measurement of $\mathrm{M}_{\text {top }}$ based on the aL distribution would be subject to a large systematic uncertainty.

As we will see in the following, the use of $\mathrm{rL}$ allows one to combine in a simple way the event structure and the information from other analyses which look for top by "tagging" beauty quarks. However, as a conclusion from this chapter, we see that one does not need to refer to any specific ISAJET prediction to see disagreement between data and QCD.

\subsubsection{Uncertainty in the calorimeter response linearity}

The jet energy resolution is typically $100 \%$ and the energy scale is known to be within $5 \%$ to $10 \%$. We found that worsening the jet energy resolution in a reasonable way has little effect on the shape of the $\mathrm{E}_{t}$ (jet) spectra. Also changing the jet energy scale by as much as $10 \%$ doesn't modify in a relevant way the shape of the predicted $E_{t}(j e t)$ spectra, in the $E_{t}(j e t)$ range of interest[88]. We try to take into account a possible non-linearity of the calorimeter by scaling down all jets at $\mathrm{E}_{t}>50 \mathrm{GeV}$ by $5 \%$ in the data events. For most of the events in the SS the value of $\ln (r L)$ decreases, but the 10 events at $\ln (r L)>0$ still have $\ln (r L)>0$.

\subsubsection{Background distribution in $\ln (\mathrm{rL})$}

It has been already noticed that the number of expected non-W background events is small. However, this background could be mostly in the region $\ln (\mathrm{r} L)>0$, where an excess of data

\footnotetext{
${ }^{4}$ In the case with no gluon radiation, the out-of-cone corrections for the jet energy have not been applied.
} 
is present. For this reason the distribution in $\ln (\mathrm{rL})$ of the non-W events has been studied. Table 5.10 uses the same notation introduced in subsection 5.2.2, and shows the number of "W" events (passing the $\not_{t}$ and $\mathrm{M}_{\text {trans }}$ cuts) and of "non-W" events in the SS sample for different values of the isolation cut. The number of events at $\ln (r L)<0$ increases from 5 to 47

\begin{tabular}{|l|cc|cc|}
\hline \multicolumn{5}{|c|}{ Signal Sample (SS) } \\
\hline \hline & \multicolumn{2}{|c|}{$\ln (\mathrm{rL})<0$} & \multicolumn{2}{c|}{$\ln (\mathrm{rL})>0$} \\
\hline & non-W & W & non-W & W \\
\hline Iso $<0.1$ & 13 & 5 & 6 & 10 \\
\hline $0.1<$ Iso $<0.5$ & 28 & 1 & 5 & 0 \\
\hline
\end{tabular}

Table 5.10: Number of $W$ and non- $W$ events at $\ln (r L)>0$ and $\ln (r L)<0$ in the top candidates enriched sample (SS), for different values of the isolation cut.

$\left(13+5+28+1\right.$ in table 5.10 in the $\ln (\mathrm{rL})<0$ column) when one releases the $\boldsymbol{E}_{t}$ and $\mathrm{M}_{\text {trans }}$ cuts, and makes the isolation cut at Iso $<0.5$. The number of events at $\ln (r \mathrm{~L})>0$ changes instead only from 10 to $21\left(6+10+5\right.$ in table 5.10 in the $\ln (\mathrm{rL})>0$ column). The distribution in $\mathrm{M}_{\text {trans }}$ and $\mathrm{P}_{t}$ (lepton) for these $47(21)$ events is shown in figure $5.8(\mathrm{a}),(\mathrm{c})(5.8(\mathrm{~b}),(\mathrm{d}))$. In the 21 events at $\ln (\mathrm{rL})>0$ a clear signal of good W's is visible. The 47 events at $\ln (\mathrm{rL})<0$ are dominated by background. There are two possible explanations for this:

a) The distribution in $\ln (\mathrm{rL})$ for the non-W background is softer than that of real QCD W's.

b) In the $\ln (\mathrm{rL})>0$ region there is a certain fraction of top events (good W's), in addition to QCD W+jet events contaminated by non-W background ( $10 \%$ circa).

Figure 5.9 (a) shows the distribution in $\ln (\mathrm{rL})$ of the 53 events which enter in the SS sample when one releases the $\mathbb{F}_{t}$ and $\mathrm{M}_{\text {tras }}$ cuts (68-15 events in table 5.10). This background enriched sample has a distribution in $\ln (\mathrm{rL})$ similar to that expected for $\mathrm{W}+\mathrm{jet} \mathrm{QCD}$ events, as predicted by VECBOS (see figure 5.5 (a)). The W signal in figures 5.8 (b) and (d) should be due to an original enrichment of the sample in good W's, coming from a source different from QCD, as for example top events. The same study has been done for the CS sample. The result is shown in table 5.11. Also the background due to WW events is distributed in a 

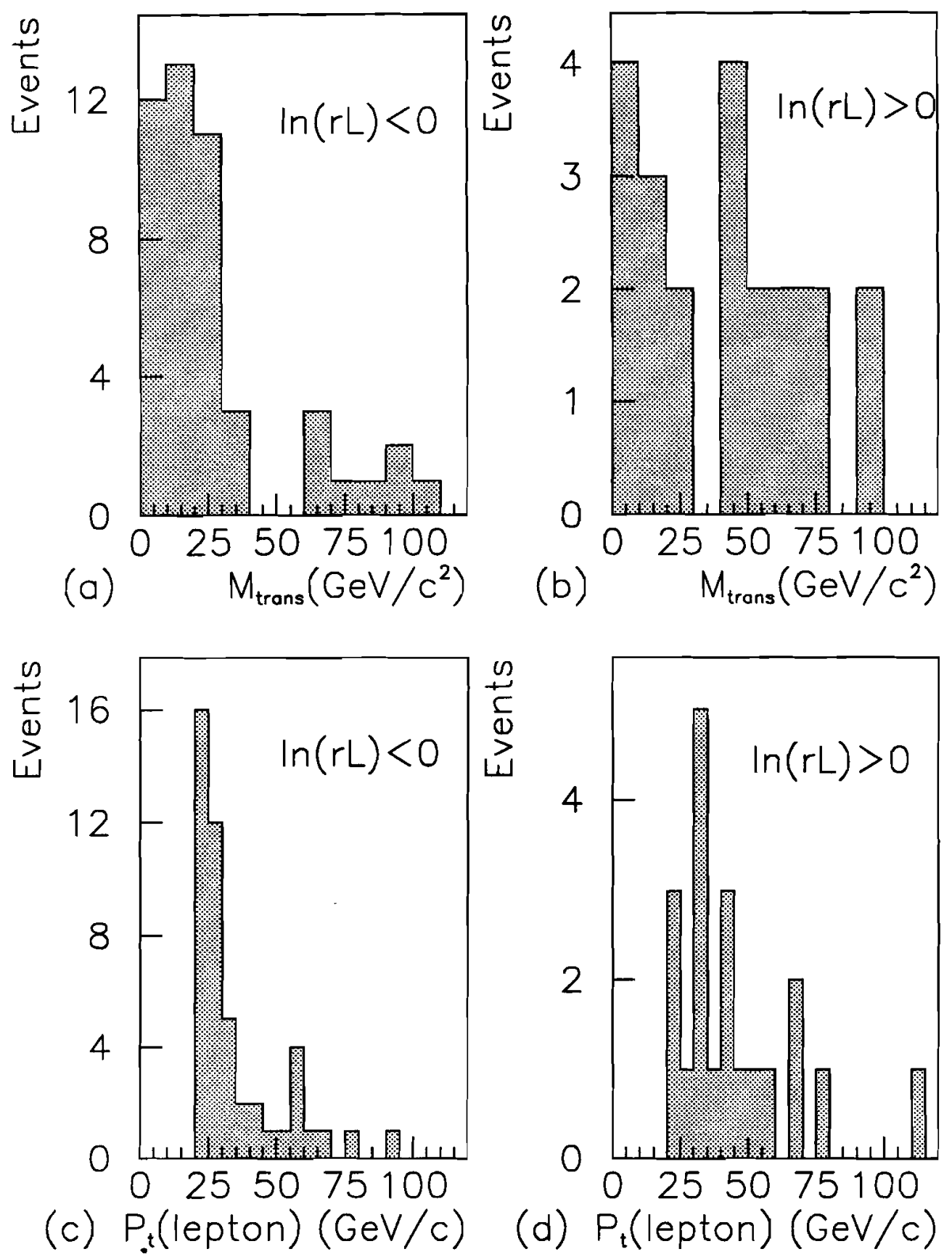

Figure 5.8: (a) Distribution in $M_{\text {trans }}$ for the 47 events at $\ln (r L)<0$ which, together with the 21 at $\ln (r L)<0$, constitute the SS sample when one releases the $\mathbb{P}_{t}$ and $M_{\text {tras }}$ cuts, and makes the isolation cut at Iso<0.5; (c) distribution in $P_{t}$ (lepton) for the same events. (b) and (d) are the corresponding distributions for the 21 events at $\ln (r L)>0$. 

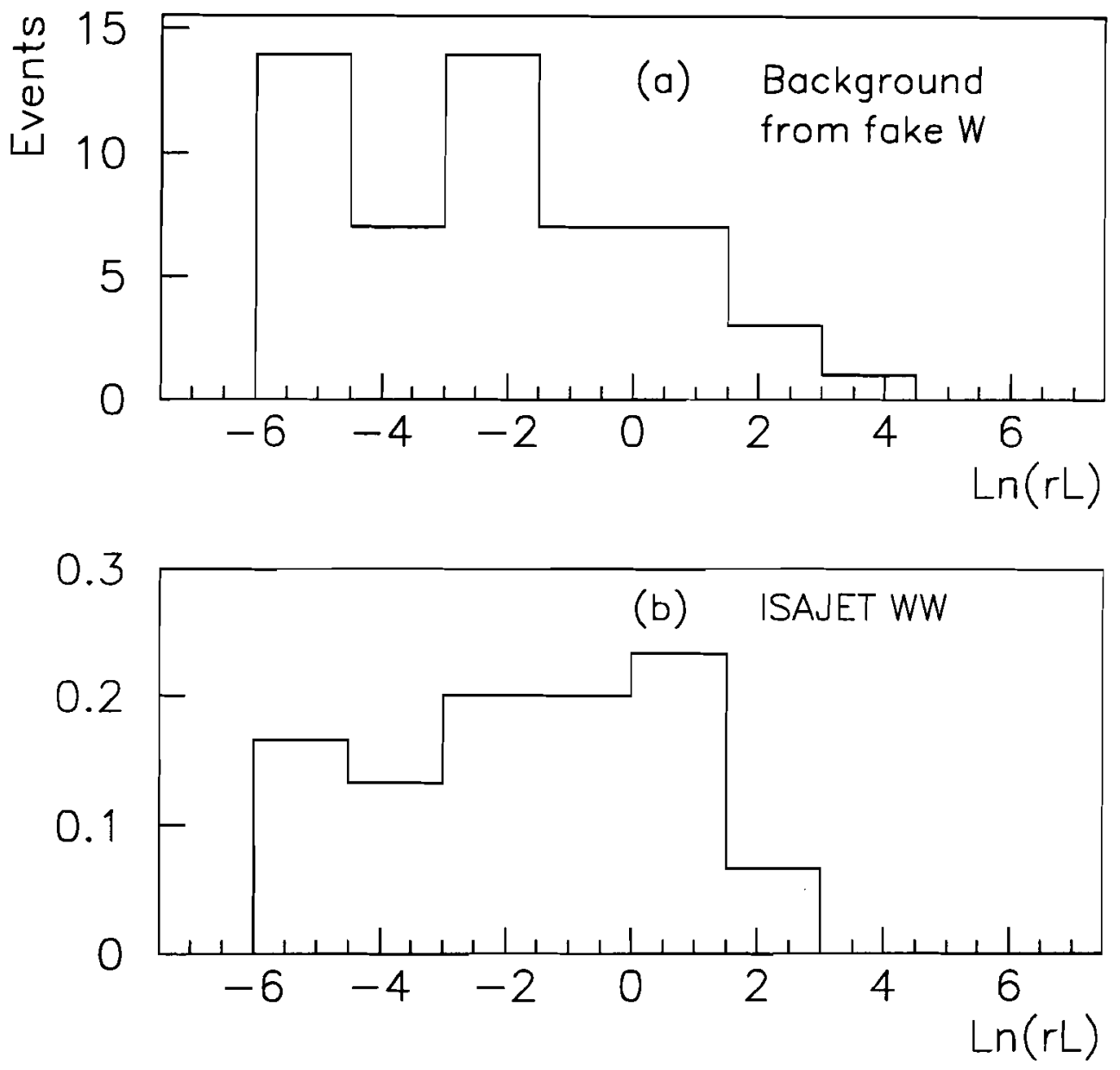

Figure 5.9: (a) Distribution in $\ln (r L)$ for the 53 events enriched in non- $W$ QCD background, which enter the SS sample when one releases the $\boldsymbol{E}_{t}$ and $M_{\text {trans }}$ cuts and makes the isolation cut at Iso $<0$; (b) distribution in $\ln (r L)$ for $W W$ events generated with ISAJET. The distributions have been normalized to 1 . 


\begin{tabular}{|c|cc|cc|}
\hline \multicolumn{4}{|c|}{ Control Sample (CS) } \\
\hline \hline & \multicolumn{2}{|c|}{$\ln (\mathrm{rL})<0$} & \multicolumn{2}{c|}{$\ln (\mathrm{rL})>0$} \\
\hline & non-W & W & non-W & W \\
\hline Iso $<0.1$ & 31 & 20 & 8 & 11 \\
\hline $0.1<$ Iso $<0.5$ & 73 & 5 & 13 & 0 \\
\hline
\end{tabular}

Table 5.11: Number of $W$ and non- $W$ events at $\ln (r L)>0$ and $\ln (r L)<0$ in the sample depleted of top candidates (CS), for different values of the isolation cut.

similar way. Only $(30 \pm 9) \%$ of the WW events, generated with the ISAJET Montecarlo (see figure $5.9(\mathrm{~b}))$ is at $\ln (\mathrm{rL})>0$.

One can therefore conclude that the non-W and di-boson background cannot explain the excess of events at $\ln (r L)>0$.

As a conclusion, from Chapter 5 we find: the observation of 10 events at $\ln (r \mathrm{~L})>0$ in the $\mathrm{SS}$ is in disagreement with VECBOS predictions for QCD $\mathrm{W}+$ jet production.

We do not find any explanation for the disagreement in terms of non-W background or WW production.

For the analysis we are doing here, namely comparing the number of events at positive and negative $\ln (\mathrm{rL})$, the jet energy uncertainties are of minor importance.

For a comparison data-VECBOS the $W+3$ jet Montecarlo should be used, because VECBOS results in an inclusive prediction[89]. But even if we compare the data to the $W+4$ jet prediction, the probability to observe 10 events is less than $1 \%$ (given a background prediction of $2.56_{-1.07}^{+1.62}$, Table 5.8).

The SS therefore must contain a new physics process, possibly top. We try to obtain more information on the nature of this new process in the following chapter. 


\section{Chapter 6}

\section{Compatibility with Top Hypothesis}

In this chapter we explore in more detail whether the excess of events at $\ln (r L)>0$ can be attributed to $t \bar{t}$ decays. This hypothesis, suggested by the jet activity, is confirmed by the presence of secondary vertices identified by the SVX, as well as by the study of the invariant masses of the candidate events. The study of the di-lepton candidates rejected from the sample is not in disagreement with this hypothesis.

\subsection{Identification of b Quarks in the Selected Events}

CDF is searching for top in the single lepton channel also with different analyses. In order to separate the top signal from the $\mathrm{W}+$ jets background these analyses try to identify $b$ quarks in the events, since there is a $b$ quark in the $t \rightarrow W b$ decay. To this end CDF developed two different techniques. The first one uses the information coming from the SVX to identify $b$ 's through the presence of tracks from displaced vertices. CDF developed three algorithms: JETVTX[90], JP[91], and DPHI[92]. Another technique (SLT[93]) identifies $b$ quarks through their semileptonic decay $b \rightarrow l$ or $b \rightarrow c \rightarrow l(l=e$ or $\mu)$, looking for a soft lepton. Table 6.1 shows for each event of the SS whether it was identified by one or more $b$-identification algorithms. The 15 events of the SS are divided into two sections in table 6.1: in the upper one are those events (10) which have at least one jet which is inside the SVX acceptance. In the lower one are the other five events. Out of 10 events which have at least one jet inside the SVX acceptance, $6(60 \%)$ have a secondary vertex. In the lower section, one of the events is 
identified by the SLT algorithm. The asterisk in the fifth column indicates that there are 4 or more jets in the event. The same table is also shown for the 31 events of the control sample

\begin{tabular}{|c|c|c|c|c|c|c|c|}
\hline Run-Event & $\ln (\mathbf{r} \mathbf{L})$ & JETVTX & JP & $\bar{D}-\bar{\phi}$ & SLT & 4th jet & lepton \\
\hline $47616-24577$ & -4.6 & & & & & & $\bar{e}$ \\
\hline $44931-59686$ & -3.9 & & & & & & $\bar{e}$ \\
\hline $42517-44047$ & -2.7 & $\bar{\phi}$ & A & $\Phi$ & $\bar{\phi}$ & & $\mu$ \\
\hline $43351-266423$ & 1. & & A & & $\bar{\varphi}$ & * & $\mu$ \\
\hline $457 \overline{79-6523}$ & 1.3 & & & & & * & $e$ \\
\hline $43336-248916$ & 1.8 & & & & & & $\mu$ \\
\hline $42539-200087$ & 2.7 & $\bar{\phi}$ & & $\boldsymbol{\phi}$ & A & * & $\mu$ \\
\hline $45753-79414$ & 2.9 & & A & & & * & $\mu$ \\
\hline $40758-44414$ & 3.3 & A & $\bar{A}$ & & & * & $e$ \\
\hline $43096-47223$ & 3.3 & A & A & A & & * & $\bar{e}$ \\
\hline Run-Event & $\ln (\mathrm{rL})$ & JETVTX & $\mathrm{JP}$ & $\mathrm{D}-\phi$ & SLT & 4th jet & lepton \\
\hline $45801-80320$ & -2.7 & & & & & & $e$ \\
\hline $45902-240098$ & -1.2 & & & & & & $e$ \\
\hline $43276-101844$ & 1.2 & & & & & & $\mu$ \\
\hline $42913-59303$ & 2.2 & & & & & & $\bar{e}$ \\
\hline $45705-54765$ & 2.4 & & & & $\bar{\phi}$ & * & $e$ \\
\hline
\end{tabular}

Table 6.1: Events of the SS sample identified by the various algorithms for the $b$ identification. The upper section of the table shows the events which have at least one jet inside the SVX acceptance. The lower section contains those events which do not have any jet inside the SVX acceptance. The asterisk in the fifth column indicates that there are \& or more jets in the event $\left(E_{t}>15 \mathrm{GeV}\right)$.

(table 6.2). In the upper section there are 19 events which have at least one jet inside the SVX acceptance; the lower section has the remaining 12 events. Among the 19, 4 (21\%) have a $b$ signal in the SVX.

These results should be compared with the number of secondary vertices expected in the SS sample, if this sample is pure QCD ${ }^{1}$. The number of secondary vertices expected can be estimated in the following way: starting from the hypothesis that all 15 events are from $Q C D$, the probability of identifying a secondary vertex in a jet is calculated for all the jets. This probability has been obtained studying an inclusive sample of QCD jet events[94], and it is a

\footnotetext{
${ }^{1}$ In this case the $b$ content (mostly from gluon splitting) is very small, but there are secondary vertices which are produced by long lived particles, different from b's, and by groups of tracks which look as if they come from a secondary vertex due to errors in their reconstruction or in the algorithms for $b$ identification.
} 


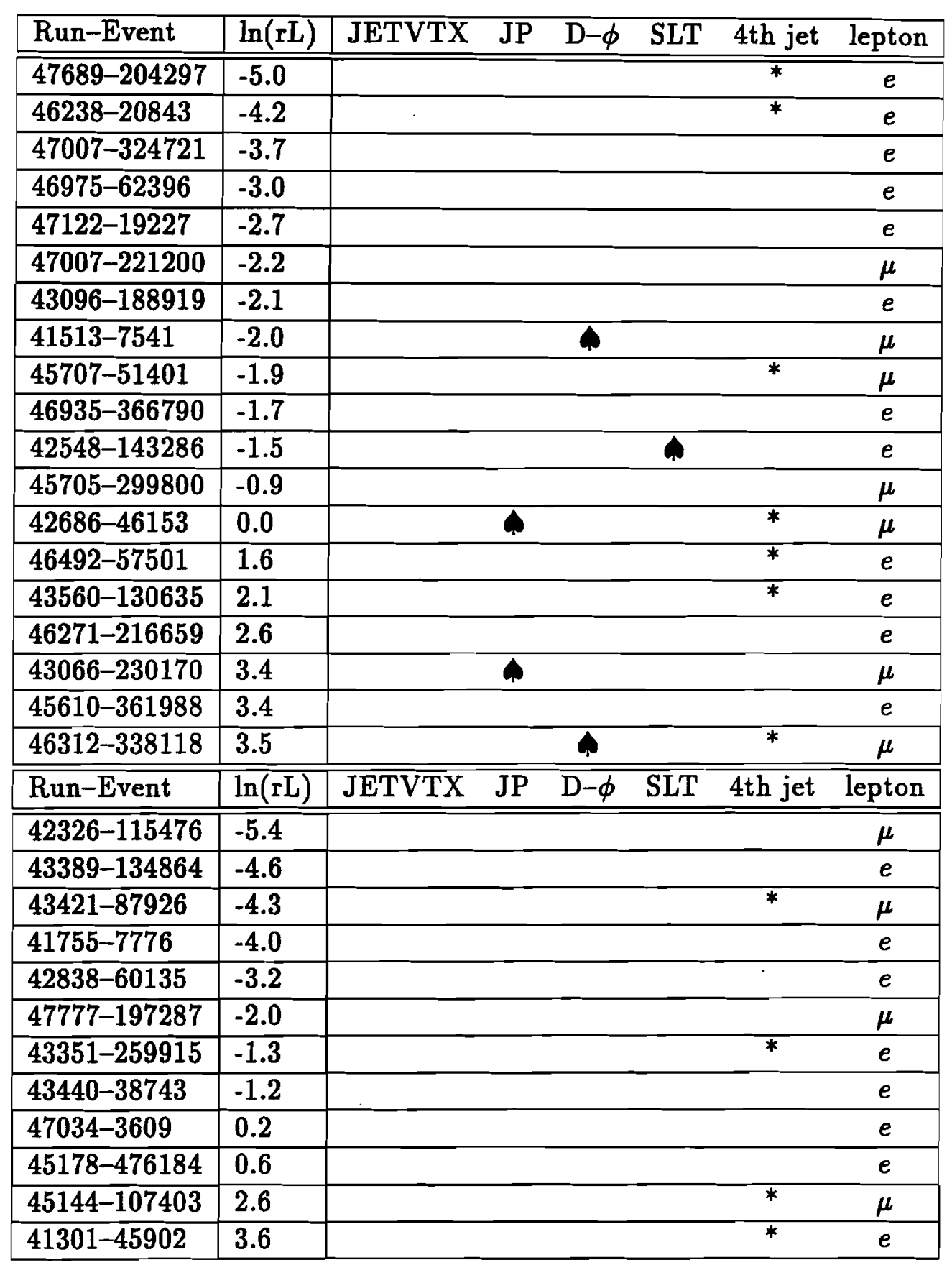

Table 6.2: Events of the CS sample identified by the various algorithms for $b$ identification. The upper section of the table shows the events which have at least one jet which is inside the $S V X$ acceptance. The lower section contains those events which do not have any jet inside the $S V X$ acceptance. The asterisk in the fifth column indicates that there are 4 or more jets in the event. 
function of the jet energy, position and charged track multiplicity. This procedure does not take into account differences in the heavy flavor production between QCD multi-jet and QCD $\mathrm{W}+$ jet events (e.g.: $\mathrm{W}+$ charm production). Presently it is assumed by the CDF SVX analysis that QCD multi-jets could rather be more enriched in $b$ 's because of the strongest direct $b$ production (while gluon splitting is assumed to be the same).

In beauty events, there are strong correlations for the various algorithms to identify the same jets. Although several algorithms are in principle more powerful than just one, these correlations must be taken into account if one wants to add the information provided by more than one algorithm. In order to ease the calculation of the significance of the observed effects, we discuss the single algorithms as follows. We consider the JETVTX algorithm first. In the SS it identifies 4 events, 1 at $\ln (r L)<0$ and 3 at $\ln (r L)>0$ (figure 6.1). Based on the jets observed in the events, one can calculate how many JETVTX identifications would be expected if they were generic QCD jets. One expects 0.64 identifications, with a statistical error of $15 \%$. Their distribution is shown in figure 6.1 (shaded area). The probability to observe 4 or more events when 0.64 are expected is of about $0.4 \%$. The CS was also studied with JETVTX, and no secondary vertices were identified. The expectation is about 1 event. The same picture emerges if one makes use of the D- $\phi$ algorithm, which identifies the smallest number of events in the SS (see table 6.1). In the SS 3 events are identified, while the expected number of identifications (for QCD jets) is 0.5 , with a statistical error of $15 \%$ (figure 6.2 (a)). The probability of observing 3 events when 0.5 are expected is $1.5 \%$. Among the 31 events of the CS, D- $\phi$ identifies 2 events, while the expectations is of 0.64 events. Their distribution in $\ln (\mathrm{rL})$ is shown in figure $6.2(\mathrm{~b})$.

The results shown in figure 6.1 and 6.2 indicate that the jet beauty content is inconsistent with QCD in the SS and marginally consistent with it in the CS. This is as expected if one supposes that $t \bar{t}$ events dominate the SS sample, and are present to a minor extent in the CS. Finally, the JP algorithm finds 5 events with a secondary vertex in the SS, the SLT algorithm identifies 3 events. As mentioned before, a full statistical analysis to work out the significance of the signal by adding all tagging informations has not been performed. However, it is not 


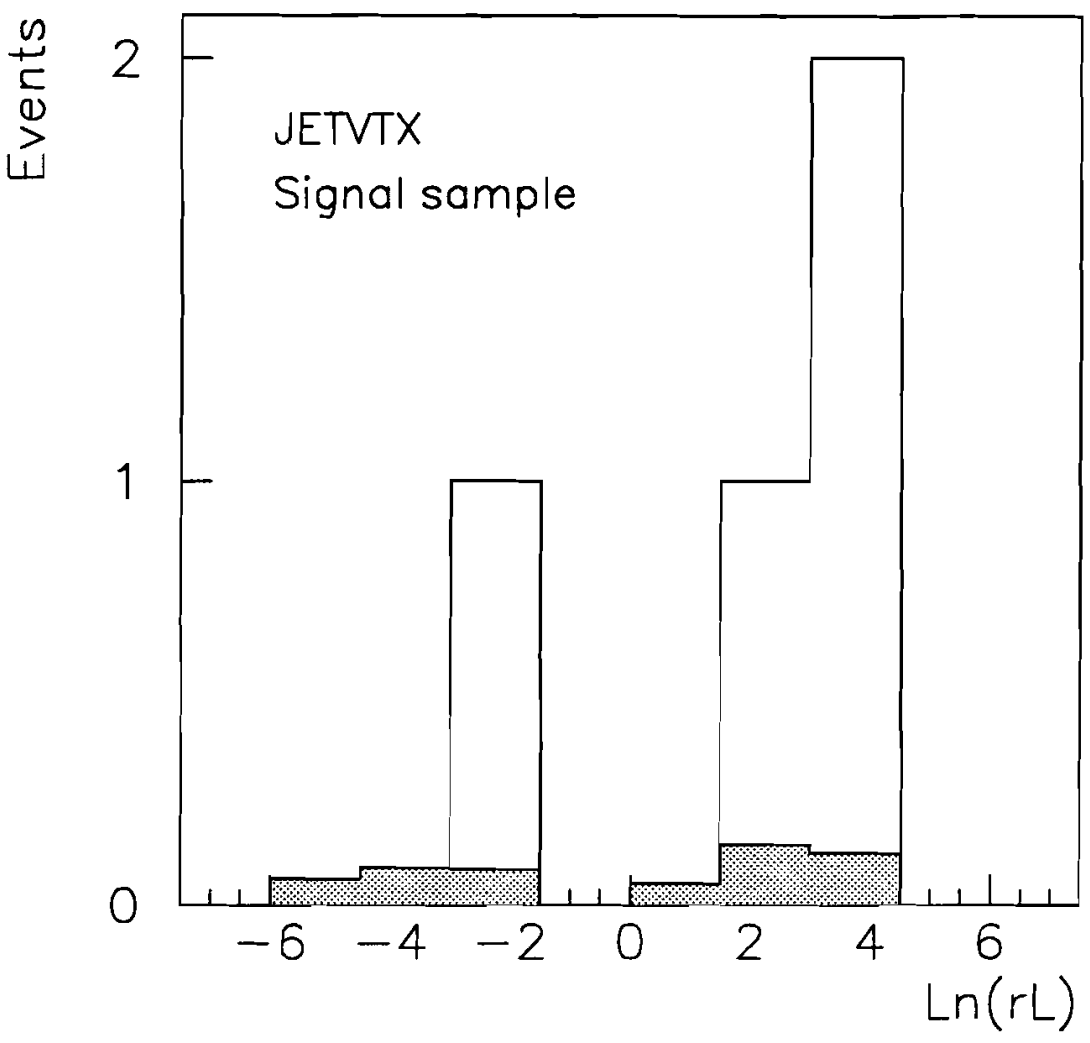

Figure 6.1: Events in the SS with a secondary vertex (JETVTX). The solid line represents the data. The shaded area shows the expected fakes of secondary vertices on the basis of the jets in the 15 events ( 0.64 events).

essential. Both the JETVTX and D- $\phi$ standalone algorithms indicate that the probability for the excess of secondary vertices to be due to the background is about $1 \%$. The probability for both JETVTX and D- $\phi$ to find a secondary vertex in $t \bar{t}$ events is about $22 \%$, after allowing for the inefficiencies for events with primary vertices outside the SVX fiducial volume. If the 15 events of the signal sample contain about 10 events from top, as indicated by the results of the kinematical analysis, the expected identification rate is 2.2 events. Clearly the $3-5$ events identified by each of the algorithms (see table 6.1) are consistent with this expectation. 

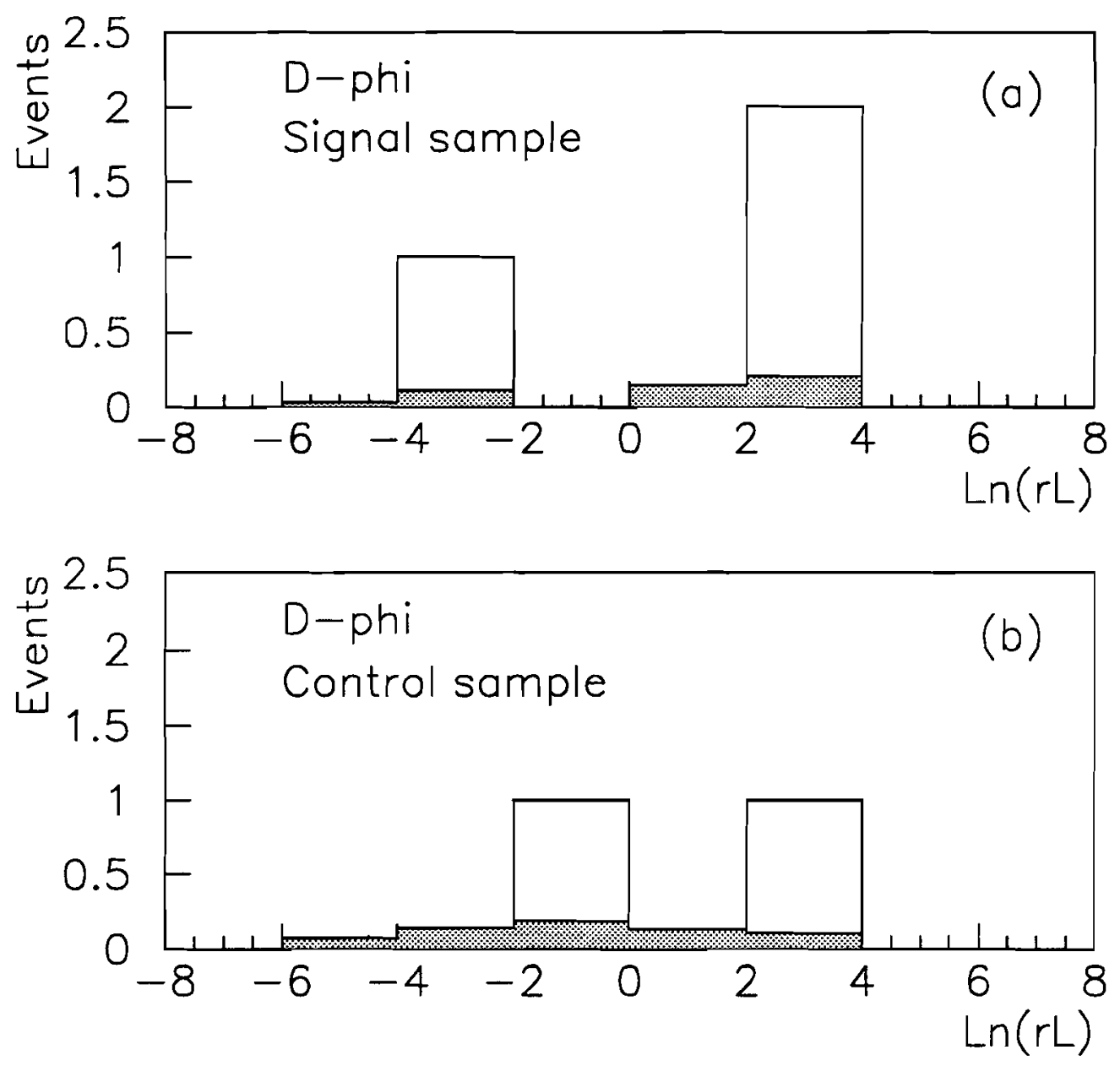

Figure 6.2: (a) Distribution in $\ln (r L)$ of the 3 events in the SS identified by the D- $\phi$ algorithm (solid line). The shaded area shows the expectation ( 0.5 events); (b) distribution in $\ln (r L)$ of the 2 events which $D-\phi$ identifies in the CS, together with the expectations (0.64 events). 


\subsection{Four Jet Events}

If one requires a fourth jet with $\mathrm{E}_{t}\left(\right.$ jet $\left._{4}\right)>15 \mathrm{GeV}$, the $\mathrm{SS}$ sample of 15 events is reduced to 7 events. Figure 6.3 (a) shows their distribution in $\ln (\mathrm{rL}$ ) (for simplicity the same functions $f_{i}$ of chapter 5 were used, see figure 5.1). The same figure also shows the expectation from VECBOS W+4 jets Montecarlo (points with error bars). VECBOS was normalized to the 5 $W+3$ jet events observed in the $S S$ at $\ln (r L)<0$. With this normalization VECBOS predicts 2.7 events with $\mathrm{E}_{t}\left(\right.$ jet $\left._{4}\right)>15 \mathrm{GeV}$, to be compared with the 7 observed. The expectation of 2.7 events should be an over-estimate, due to the normalization which has been used. The excess is consistent with the hypothesis that these 7 events are mostly top. According to ISAJET about $80 \%$ of top events $\left(M_{\text {top }}=170 \mathrm{GeV} / \mathrm{c}^{2}\right)$ will have a fourth jet with $\mathrm{E}_{t}\left(\mathrm{jet}_{4}\right)>15 \mathrm{GeV}$. In 3 out of the 7 events a secondary vertex was found by the JETVTX algorithm. The distribution of these 3 events in $\ln (\mathrm{rL})$ is shown in figure $6.3(\mathrm{~b})$. For JETVTX on the basis of the observed characteristics of the jets in the 7 events, one expects 0.15 fakes, distributed as shown in figure 6.3 (b) (shaded area). Figure 6.3 (c) shows the distribution in $\ln (\mathrm{rL})$ of the 5 events which are selected by at least one of the three SVX algorithms for the b identification. In the figure, the rate of fakes for the JETVTX algorithm was multiplied by three ( 0.45 events in total) to account approximately for the three algorithms. The chance of identifying 3 secondary vertices when 0.15 are expected, as well as 5 when 0.45 are expected, is very small. One observes that requiring a fourth jet improves the signal/background ratio. In other words, the process which was indicated by an excess of secondary vertices is also characterized by the presence of a fourth jet. This is as expected for $t \bar{t}$ events.

\subsection{Di-lepton Candidates}

If the top exists and it decays according to the Standard Model, the observations in the dilepton channel and in the single lepton+jets channel should be consistent and confirm each other. This has been checked in the selected events. Di-lepton events which have as a second lepton an $e$ or a $\tau$ would enter in the SS or CS samples because the second lepton shows up as 

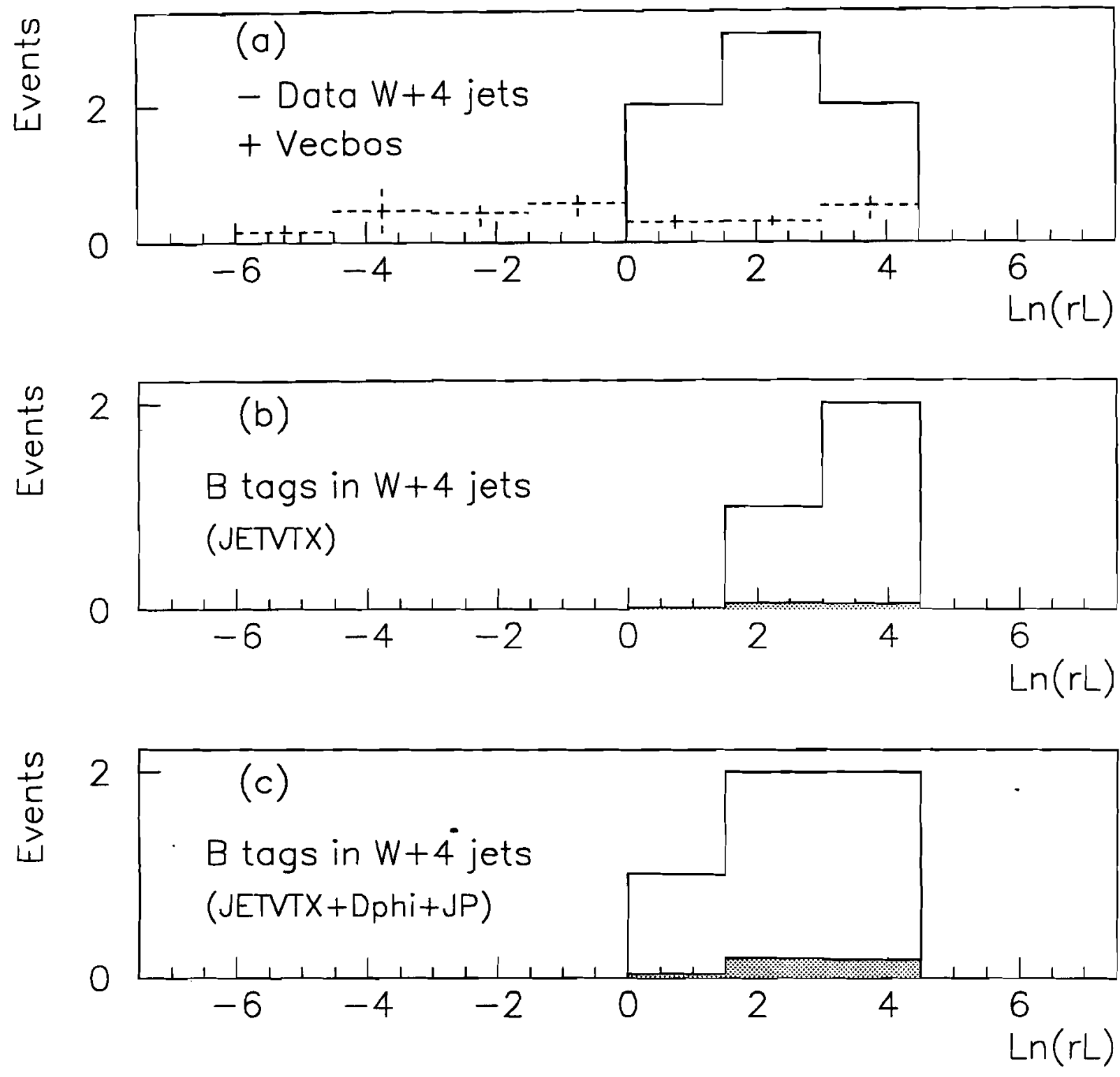

Figure 6.3: (a) Distribution in $\ln (r L)$ for the 7 events in the $S S$ with a fourth jet (solid line). Also shown is the VECBOS prediction (points with error bars); (b) Distribution in $\ln (r L)$ for the 3 events with a JETVTX secondary vertex. The shaded area superimposed shows the prediction based on the observed jets in the 7 events ( 0.15 events). (c) Distribution in $\ln (r L)$ for the 5 events tagged by at least one of the three SVX algorithms. Again the shaded area shows the prediction ( 0.45 events). 
a jet in the calorimeter. These events have been removed by an explicit veto against di-lepton candidates, described in chapter 3 (essentially any event which has in addition to the primary lepton an isolated and high $P_{t}$ track which points to a jet[73]). A study of QCD jets showed that only $1 \%$ of the jets contain such an isolated track[73].

One event was removed from the SS sample by the isolated track rejection. It is event 46935384578 , with $\ln (\mathrm{rL})=-1.3$. The track is pointing to a narrow jet and could be from a one prong $\tau$ decay. On the basis of the jets in the SS sample, one would expect 0.5 events with a jet containing a high $\mathrm{P}_{t}$ isolated track. However, one can instead start from the hypothesis that only about 6-7 out of the 15 events are from QCD production. This would leave us with an expectation of 0.2 events from QCD jet fluctuations. The relative rate of di-lepton/single lepton events from top in the SS sample should be about 15\% (according to ISAJET prediction][73]. One would therefore expect 1.5 di-lepton events from top, if there are about 10 top events in the SS sample. The fact that the event has $\ln (r \mathrm{~L})=-1.3$ is not a very strong argument against the top hypothesis, since di-lepton events should have less jet activity and therefore softer $\mathrm{E}_{t}(\mathrm{jet})$ spectra[73]. In the CS sample the expectation for high $P_{t}$ isolated tracks from QCD jet fluctuation becomes about 1 event. Also in the CS sample one di-lepton top event is expected. Several di-lepton candidates were found in the CS and rejected from it:

(1) $46818-218944, \ln (\mathrm{rL})=-2.7$

(2) $43139-284949, \ln (\mathrm{rL})=-1.9$

(3) $47311-71056, \ln (r L)=-3.9$

(4) $42727-81408, \ln (\mathrm{rL})=1.3$

(5) $45880-31838, \ln (r \mathrm{~L})=1.8$

(6) $45047-104393, \ln (r \mathrm{~L})=1.2$

From studying in detail the events it has been found that (1) and (3) are possible Drell-Yan or $\mathrm{Z}^{0}$ candidates. Events (2) and (4) cannot be classified on an individual base. Event (5) has 
been identified by the SLT algorithm. Event (6) is tagged by all of the SVX b-tag algorithms. This event is very interesting, and it has been extensively studied in[73]. One can therefore conclude that the study of di-lepton events rejected from the SS and CS samples is not in disagreement with the hypothesis that in the above samples there are some top events.

\subsection{Reconstruction of the $t \bar{t}$ Mass}

We tested[95] whether the kinematic of the events from the SS+CS samples is compatible with $t \bar{t}$ production and decay into two real W's as follows: events with an additional fourth jet with $\mathrm{E}_{t}(\mathrm{jet})>15 \mathrm{GeV}$ have been required. In order to avoid the uncertainties from modelling gluon radiation (see section 4.2 ) and also to decrease combinatorial background events with $E_{t}\left(j t_{5}\right)>10 \mathrm{GeV}$ have been rejected. Four events (three from the SS and one from the CS) out of 46 fulfill these requirements ${ }^{2}$. The mass of the lepton- $F_{t}$ system has been constrained to the $\mathrm{W}$ mass, giving two solutions with different longitudinal components of the missing $\mathrm{E}_{\boldsymbol{t}}$. In general eight combinations are obtained by associating each jet with both of these $\mathrm{W}$ mass solutions. For each combination a $\chi^{2}[95]$ is calculated based on two quantities:

1. How well the $\mathrm{W}+$ jet mass agrees with the mass of the remaining 3 -jets system;

2. How well the best 2-jets combination in the 3 -jets system agrees with the $\mathrm{W}$ mass.

Montecarlo studies[95] show that events passing the cuts of this analysis have an acceptable solution passing a loose $\chi^{2}$ cut more than $90 \%(80 \%)$ of the time for $t \bar{t}$ (QCD W+jet) events. For each combination a corresponding mass has been calculated that is the average of the $\mathrm{W}+\mathrm{jet}$ mass and the 3 -jets mass. The preferred top mass for each event is the weighted average of the masses of those combinations that pass the loose $\chi^{2}$ cut. Figure 6.4 (a) shows the distribution of the preferred mass for the 3 events in the SS and the VECBOS W+4 jets prediction. Figure 6.4 (b) shows the same 3 events compared with the ISAJET prediction, for a top mass of $170 \mathrm{GeV} / \mathrm{c}^{2}$. Figures 6.4 show the one event in the CS, compared with the VECBOS (c) and ISAJET (d) predictions. In each figure the predictions are normalized to

\footnotetext{
${ }^{2}$ These 4 events have a $\ln (\mathrm{rL})>0$. In the 3 events which belong to the SS there is a $b$ candidate.
} 

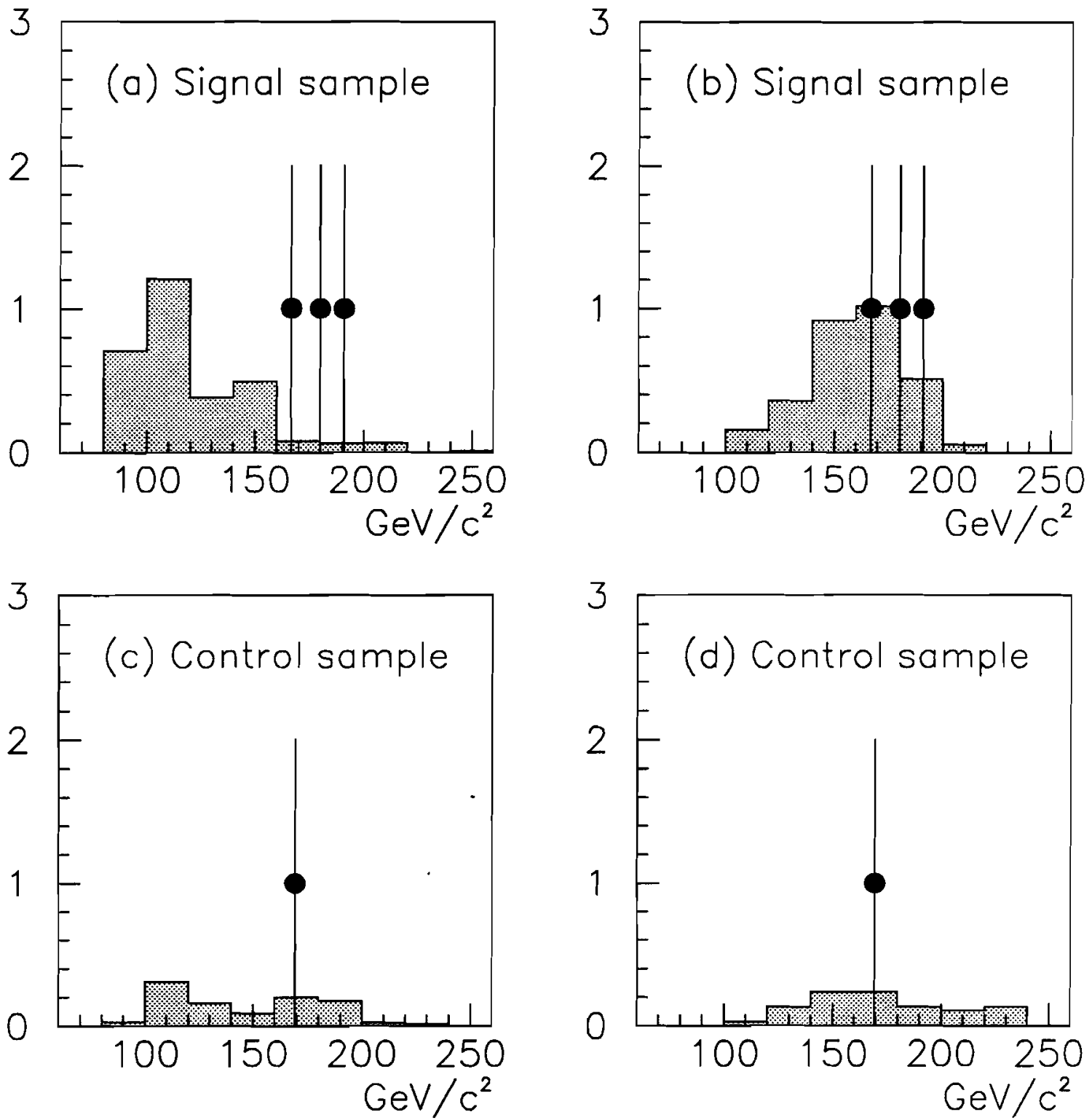

Figure 6.4: Figures on the left: distribution of the expected mass for $W+4$ jet Montecarlo events, analyzed as they were $t \bar{t}$ events (histogram), for the SS (a) and the CS (c). Figures on the right: distribution of the preferred mass for $t \bar{t}\left(M_{\text {top }}=170 \mathrm{GeV} / \mathrm{c}^{2}\right)$ Montecarlo events, analyzed as $t \bar{t}$ for the $S S(b)$ and the $C S(d)$. The \& events which allow the mass reconstruction are shown as points with errors of $100 \%$. 
the data.

The data agree with the ISAJET prediction, and not with the VECBOS prediction. In order to obtain an absolute measurement of the top mass, one would need to study carefully various experimental and theoretical uncertainties. This should be subject of future studies. Here one wants to point out that the 4 data events are compatible with the top hypothesis and that they are found at a common mass value.

\subsection{Conclusions}

- Following the analysis strategy explained in ref[45] a signal sample (SS) of 15 events with a W and 3 central jets has been selected. Based on QCD calculations for top production, it is found that the SS sample should be characterized by a signal/background ratio of about 1 , if top exists and if it has a mass around $150 \mathrm{GeV} / \mathrm{c}^{2}$.

- The $\mathrm{E}_{t}$ jet spectra of the 15 events in the SS sample are very hard, not consistent with QCD predictions. Using a relative likelihood method the 15 events were compared with top and QCD predictions. 10 events have been found to fit the top hypothesis and 5 events were found to fit the QCD hypothesis better. After taking the systematic and statistical errors in the Montecarlo simulations into account, we find that the probability that these 10 events are the result of a fluctuation of the QCD background is less than $1 \%$.

- A control sample (CS) of $31 \mathrm{~W}+3$ jet events is much better described by QCD. Also W+2 jet events are well described by QCD.

- Having gained at this point enough confidence in the presence in the SS of events coming from a new process, we tried to confirm the hypothesis that these events are from top. Clear evidence is found for an excess of $b$ quarks. This excess has a probability of $1 \%$ of being a statistical fluctuation. 7 out of the 15 events in the SS sample have at least one fourth jet with large $E_{t}$. In 6 of these events a $b$ quark has been identified by at least one of the four algorithms used (JETVTX, JP, D- $\phi$ and SLT).

It was possible to reconstruct the mass in a subsample of the total of 46 events, by requiring events which contain a fourth jet with $E_{t}>15 \mathrm{GeV}$ and do not have a fifth jet with $E_{t}>10$ 
$\mathrm{GeV}$. The 4 events selected in this way have been reconstructed following the hypothesis that they are top. A common mass is found ${ }^{3}$.

Within the Standard Model, a heavy top quark is the only source which can account for these observations.

\subsection{Future Prospects}

The sample of events is large enough, and the systematic errors are small enough to conclude that we have a new signal. However, some parts of the analysis can be further improved. For example it will be interesting to proceed in the quantitative study of the top mass. In this thesis the values obtained for $M_{\text {top }}$ from the 4 selected events are around $165-185 \mathrm{GeV} / \mathrm{c}^{2}$. However, the systematic errors have not yet been evaluated.

At the end of 1993, CDF started a new run (Ib) which should allow the integration of $100 \mathrm{pb}^{-1}$ in the next one or two years, that is, a statistics 4 times larger than that presently available. Since the event structure study is dominated by the statistical error, the increased luminosity will improve the analysis. New prospects will be open: it will be possible to compare the rate of events in the lepton+jets and in the di-leptons channels, as well as to explore new mechanisms of top production and decay, outside the SM predictions. As soon as a larger sample of certified events is available, one will be able to investigate the V-A coupling of the W.

It must then be pointed out that the nucleus of the analysis described in this thesis is the requirement of central jets. This requirement is very general and any new particle which is produced and decays centrally could be selected by our cuts. However, together with these exciting prospects, new problems will come up. The new run will have a luminosity higher than before. The number of events containing two superimposed interactions will increase. This may worsen the missing energy and the jet energy resolution. Finally, the increase of

\footnotetext{
${ }^{3} \mathrm{~A}$ precise measurement of the top mass is not attemped at this time. An estimate indicates $165<\mathrm{M}_{\text {top }}<185$ $\mathrm{GeV} / \mathrm{c}^{2}$. We expect the main uncertainties to come from the calorimeter energy scale and from initial and final state gluon radiation.
} 
the available statistics will make it necessary to perform new and more detailed Montecarlo studies. 


\section{Appendix A}

\section{Effects of the CMX Trigger on $\mathrm{W}+$ jet Events}

The central electron and muon (CMU) triggers, were highly efficient during the 1992-93 run (better than $90 \%$ and $80 \%$ respectively). However, the CMX trigger had problems, so that for about $83 \%$ of the run, the level 1 single tower calorimeter trigger was required in addition to the CMX muon candidate. CDF did detailed studies on this trigger[96], and some of these studies have been directly connected with the top search [97].

In the context of the analysis described in this thesis an empirical study has been done, based only on the data, in order to check if a fake "signal" can be produced due to trigger problems. For this purpose a muon sample and an electron sample have been selected requiring that the leptons be restricted to the rapidity region covered by the CMX system (see subsection 2.2.3). To point out possible problems due to inefficiency, no specific requirement on a particular trigger has been made. No cut on $\boldsymbol{F}_{t}$ has been applied.

The samples contain 1700 muons and 5655 electrons. $53 \%$ of the muons, and only $34 \%$ of the electrons have at least one jet with $\mathrm{E}_{t}>15 \mathrm{GeV}$. This imbalance shows that the CMX trigger inefficiency caused the loss of those events which had a reduced probability to activate any trigger besides the muon trigger (as an example, events without jets). In events with jet activity, the CMX trigger inefficiency becomes less important.

In order to understand when the effect of this inefficiency becomes negligible, we measured the electron/muon ratio as a function of jet activity in the events. The results are summarized 
in table A.1. One observes a considerable increase in the relative number of muons when at

\begin{tabular}{|l|c|c|c|}
\hline Sample & $e$ & $\mu$ & $e / \mu$ \\
\hline \hline $\mathrm{E}_{t}\left(\right.$ jet $\left._{1}\right)<15 \mathrm{GeV}$ & 3151 & 804 & 3.92 \\
\hline $\mathrm{E}_{t}\left(\right.$ jet $\left._{1}\right)>15 \mathrm{GeV}$ & 2488 & 895 & 2.78 \\
\hline $\mathrm{E}_{t}\left(\right.$ jet $\left._{2}\right)>15 \mathrm{GeV}$ & 492 & 203 & 2.43 \\
\hline $\mathrm{E}_{t}\left(\right.$ jet $\left._{3}\right)>15 \mathrm{GeV}$ & 123 & 46 & 2.67 \\
\hline
\end{tabular}

Table A.1: Ratio between electrons and muons for events characterized by a different jet activity.

least one jet is required, with respect to the case in which there are no jets at all.

If two jets are required, then the relative number of muons increases further, but only by $\mathrm{O}(10 \%)$. To require a third jet should have an even smaller effect. What one sees instead, is that the relative fraction of muons decreases by a little, but this effect is within a standard deviation. Figure A.1 (a) shows the $E_{t}\left(\right.$ jet $\left._{1}\right)$ distribution for those electrons (dashed line) and muons (points with error bars) which have at least one jet with $\mathrm{E}_{t}>15 \mathrm{GeV}$. Muons and electrons are normalized to the same number. The jet spectra in the muon sample look harder: the trigger efficiency is clearly correlated to the hadronic activity (namely to the jet energies and multiplicities) in the events. Such an effect would make it difficult to study $\mathrm{W}+1$ jet events with CMX muons. Figure A.1 (b) compares the $E_{t}\left(\right.$ jet $\left._{2}\right)$ spectra for electrons and muons (normalized to the same number) with at least two jets with $E_{t}>15 \mathrm{GeV}$; one cannot see any significant difference. One can therefore conclude that when 3 jets with $\mathrm{E}_{t}>15 \mathrm{GeV}$ are required, the spectra of the associated jets studied in this analysis are not sensitive to the CMX trigger inefficiency.

The same argument can also be applied to the CMU trigger, which is even less inefficient. In our sample of 15 events, selected by requiring 3 jets with $E_{t}>20 \mathrm{GeV}$, there are two events in which the muon trigger has been activated by a muon which is not the primary one. These events are 43336-248916 and 45753-79414. The first one is a CMU muon; this event activated the trigger which requires two muons, and in addition 4 other triggers. The other one is a CMX muon; the muon trigger was activated by a CMU muon candidate, and there are three 

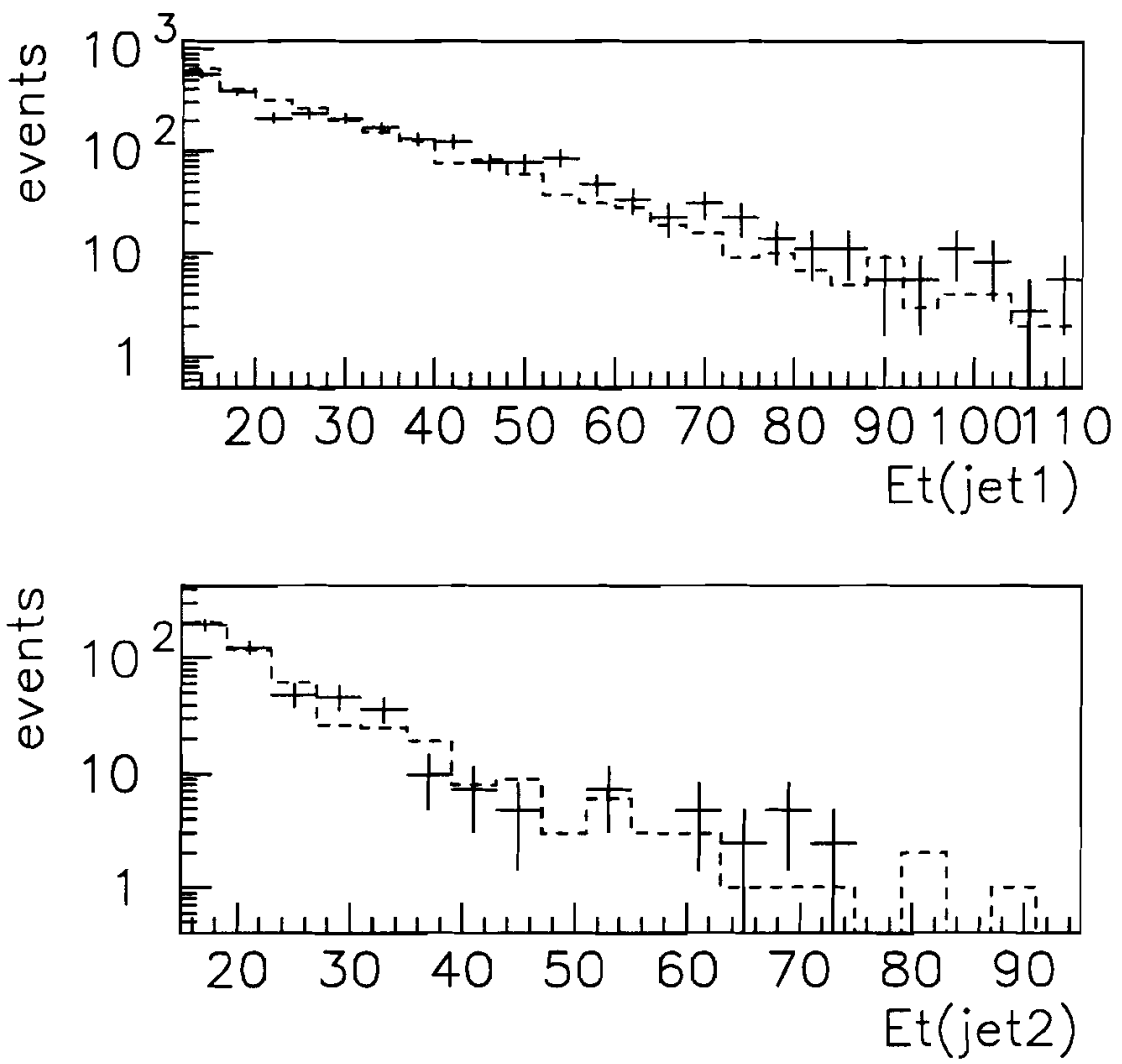

Figure A.1: (a) $E_{t}$ (jet $1_{1}$ ) distributions for electrons (dashed line) and muons (points with error bars) which have at least one jet with $E_{t}>15 \mathrm{GeV}$; (b) $E_{t}$ (jet $\left.t_{2}\right)$ distributions for electrons and muons which have at least two jets with $E_{t}>15 \mathrm{GeV}$. Muons and electrons are normalized to the same number. 
more triggers ${ }^{1}$. Following these remarks, the two events have not been removed.

${ }^{1}$ Studying a sample of $W+3$ jet events, it was noticed that on average there are 3 triggers activated for each event, in addition to the muon trigger. In (95 \pm 5$) \%$ of the events there is at least one additional trigger besides the muon trigger. 


\section{Appendix B}

\section{Choice of Kinematical Cuts}

The cuts which defined the SS and CS samples were not chosen in order to isolate a fake signal which disappears as soon as the cuts are slightly modified ("fine tuning"). In this appendix we summarize briefly how the event structure analysis was developed: that is how we arrived at the particular set of cuts used in this thesis.

The event structure analysis consists of two different parts:

1. First of all we require a $\mathrm{W}$ candidate, starting from our official CDF data samples and isolating the $\mathrm{W}$ with a set of cuts commonly used by our collaboration. This is a standard procedure, and will not be included in the following discussion.

2. We then require at least 3 jets with $\mathrm{E}_{t}>20 \mathrm{GeV}, \mid \eta($ jet $) \mid<2$ and $\mid \cos \theta^{*}($ jet $) \mid<0.7$. These are the cuts which isolate the signal. Different cuts were used in previous stages of the analysis.

The analysis and a preliminary definition of the cuts, suggested by a comparative study of top and QCD W+jet background Montecarlo events, were initially as described in[45] (see Chapter 1). The analysis was applied to the data collected in 88-89. In the beginning the $\mathrm{E}_{t}(\mathrm{jet})$ cuts were very loose, since our analysis started with a search for a low mass top, $\sim 100 \mathrm{GeV} / \mathrm{c}^{2}$. We required 3 jets with $E_{t}\left(\right.$ jet $\left._{1,2,3}\right)>15 \mathrm{GeV}$ and $\mid \eta\left(\right.$ jet $\left._{1,2,3}\right) \mid<1.5$ Since the Montecarlo predicts that there are few top events with $15 \mathrm{GeV}<\mathrm{E}_{t}\left(\right.$ jet $\left._{1}\right)<30 \mathrm{GeV}$, we in addition required $\mathrm{E}_{t}\left(\right.$ jet $\left._{1}\right)>30$ $\mathrm{GeV}$. The $\left|\cos \theta^{*}(\mathrm{jet})\right|$ cut was at 0.8 , since this value was suggested to be a reasonable choice by the Montecarlo studies in[45]. 


\begin{tabular}{|c|ccccc|}
\hline Run-Event & $\begin{array}{c}\mathrm{E}_{t}\left(\text { jet }_{1}\right) \\
\mid \cos \theta^{*}\left(\text { jet }_{1}\right) \mid\end{array}$ & $\begin{array}{c}\mathrm{E}_{t}\left(\text { jet }_{2}\right) \\
\mid \cos \theta^{*}\left(\text { jet }_{2}\right) \mid\end{array}$ & $\begin{array}{c}\mathrm{E}_{t}\left(\text { jet }_{3}\right) \\
\mid \cos \theta^{*}\left(\text { jet }_{3}\right) \mid\end{array}$ & $\begin{array}{c}\mathrm{E}_{t}\left(\mathrm{jet}_{4}\right) \\
\mid \cos \theta^{*}\left(\text { jet }_{4}\right) \mid\end{array}$ & $\overline{\mathrm{P}}_{t}(\mathrm{~W})$ \\
\hline \hline $20433-27043$ & 62. & 27. & 17. & 5. & 83. \\
& 0.05 & 0.69 & 0.42 & 0.99 & \\
\hline $18098-18655$ & 39. & 37. & 33. & 29. & 96. \\
& 0.3 & 0.43 & 0.07 & 0.46 & \\
\hline $19250-35054$ & 112. & 62. & 30. & 20. & 113. \\
& 0.68 & 0.44 & 0.27 & 0.63 & \\
\hline \hline $20015-20566$ & 57. & 55. & 47. & 22. & 48. \\
& 0.01 & 0.47 & 0.69 & 0.85 & \\
\hline
\end{tabular}

Table B.1: Events from the 88-89 run which passed the selection. The first 3 are electrons, the last one is a muon.

Four events from the 88-89 run pass this selection. Some of their parameters are shown in table B.1. These 4 events were carefully examined. They appear to be of good quality and 2 of them have a non isolated soft lepton candidate[98]. They also have high $\mathrm{E}_{t}$ jets. In CDF note \# 1912 (August 1992) we presented a quantitative evaluation of this observation, including a study of systematic errors and theoretical uncertainties. In order to define a relative and an absolute likelihood, 3 variables have been used: $E_{t}\left(\right.$ jet $\left._{3}\right), E_{t}\left(\right.$ jet $\left._{4}\right)$ and $P_{t}\left(\text { jet }_{1}+\text { jet }_{2}\right)^{1}$. Based on the absolute likelihood (computed with respect to the QCD predictions), we found a probability of less than $1 \%$ for having observed a statistical fluctuation of QCD events, taking into account systematic errors and uncertainties. The kinematical properties of the events strongly suggested a top mass much above $100 \mathrm{GeV} / \mathrm{c}^{2}$. Three out of the four selected events also survive the kinematical cuts described in this thesis. The observation of two or three top candidates in the $88-89$ data $\left(4 \mathrm{pb}^{-1}\right)$ is consistent with the observation of about 10 candidates in the 92-93 data $\left(21 \mathrm{pb}^{-1}\right)$.

Next, the $\mathrm{E}_{t}\left(\mathrm{jet}_{4}\right)$ variable, which has a high signal/background separation power, has been abandoned since it has been suggested that the analysis would be easier to understand if we refrain from using $E_{t}\left(\right.$ jet $\left._{4}\right)$. In a preliminary study of the 92-93 W data, and in particular of the associated jet spectra, we found that jets were systematically higher in Et by about 1-2

\footnotetext{
${ }^{1}$ The original idea was to use $P_{t}(W)$, but the neutrino reconstruction was controversial, so it was difficult to agree on a systematic error. It was therefore decided to use a similar variable, $P_{t}\left(\right.$ jet $_{1}+$ jet $\left._{2}\right)$.
} 
$\mathrm{GeV}$, and we found evidence that the increased luminosity and the increased rate of double interactions had caused this shift (see CDF note \# 2056). This conclusion has been confirmed by the observation that the energy assigned to the jets was more sensitive to the size of the jet reconstruction cone. Following these observations, we decided to use a cone of radius 0.4 instead of 0.7 , and to raise the $E_{t}$ threshold to $20 \mathrm{GeV}$ for jet ${ }_{2}$ and jet $t_{3}$ and to $50 \mathrm{GeV}$ for jet $_{1}$. Also in this case there was' a signal, with the same charateristics of the one found in the 88-89 data (CDF note \# 2142, June 1993). In this study different definitions of the likelihood have been used; the results did not change. During 1993 the results of the analyses which search for top by identifying $b$ 's in the event came out. At this point it was important to add all the pieces of information together. Both these analyses apply a cut on $\mathrm{E}_{t}$ (jet) at $15 \mathrm{GeV}$ (before having applied the corrections described in Chapter 3 ) and require that $\mid \eta($ jets $) \mid<2$. To make our cuts as similar as possible to the cuts applied by these analyses, we therefore cut on the (corrected) $\mathrm{E}_{t}(\mathrm{jet})$ at $20 \mathrm{GeV}^{2}$ and on $\eta(\mathrm{jet}) \mid$ at 2 . The $\left|\cos \theta^{*}\right|$ cut has been tightened from 0.8 to 0.7 , in order to reduce the higher background which was introduced by softening the cut on $\mathrm{E}_{t}\left(\right.$ jet $\left._{1}\right)$.

The changes made in the last two years are therefore justified. The first changes have been made in order to reduce the new systematic uncertainties from multiple interactions. The other changes have been made to make our analysis as similar as possible to the other analyses developed at CDF for the top search. In all the different stages of the analysis the results were consistent.

\footnotetext{
${ }^{2}$ This cut is on average not really different from a cut at $15 \mathrm{GeV}$ on the corrected energy.
} 
$-$

$-$

$-$

$-$ 


\section{Bibliography}

[1] P.W. Higgs, Phys. Lett. 12, 132 (1964); Phys. Rev. 145, 1156 (1966).

[2] Particle Data Group, Phys. Rev. D 45 (1992).

[3] S.L. Glashow, Nucl. Phys. 22, 579 (1961);

S. Weinberg, Phys. Rev. Lett. 19, 1264 (1967);

A. Salam, "Elementary Particle Theory" (Nobel Symposium n. 8), edito da Svartholm (Almquist and Wiksell, Stockholm, 1968) p. 367;

S.L. Glashow, J. Iliopoulos, and L. Maiani, Phys. Rev. D 2, 1285 (1970).

[4] C.N. Yang and R.L. Mills, Phys. Rev. 96, 191 (1954);

M. Gell-Mann, “Acta Physica Austriaca, Suppl.”, IX, 733 (1972);

H. Fritzsch and M. Gell-Mann, XVI International Conference High Energy Physics, Batavia, Vol. II p.135 (1972);

H. Fritzsch, M. Gell-Mann and H. Leutwyler, Phys. Lett. B 47, 365 (1973).

[5] M. Kobayashi and K. Maskawa, Progr. Theor. Phys. 49, 652 (1973).

[6] G. Altarelli, G. Parisi, Nucl. Phys. B 126, 298 (1977)

[7] UA2 Collaboration, Phys. Lett. 268 B, 145 (1992)

[8] S.D. Ellis, Z. Kunszt, and D.E. Soper, Phys. Rev. Lett. 64, 2121 (1991).

[9] CDF Collaboration, Phys. Rev. Lett. 68, 1104 (1992);

CDF Collaboration, Phys. Rev. Lett. 70, 1376 (1993). 
[10] CDF Collaboration, Phys. Rev. D 48, 998 (1993).

[11] CDF Collaboration, Phys. Rev. D 45, 1448 (1992);

CDF Collaboration, Phys. Rev. Lett. 69, 2896 (1992).

[12] CDF Collaboration, Phys. Rev. Lett. 71, 500 (1993).

[13] S.L. Glashow, J. Iliopoulos, L. Maiani, Phys. Rev. D 21285 (1970).

[14] G.L. Kane and M. Peskin, Nucl. Phys. and Met. B 195, 29 (1985).

[15] CLEO Collaboration, Phys. Rev. D 35, 3533 (1987).

[16] M. K. Gaillarde, B. W. Lee, Phys. Rev. D 10, 897 (1974); D.P. Roy, S.U. Sankar, Phys. Lett. B 243, 296 (1990).

[17] CDF Collaboration, Phys. Rev. Lett. D 67, 3351 (1991);

ALEPH Collaboration, internal note 93-132 PHYSIC 93-113.

[18] W. Bartel et al, Phys. Lett. B 146, 437 (1984).

[19] G. Kane, Winskonsin Top Symposium (1992).

[20] D. P. Roy, S.U. Sankar, Phys. Lett. B 243, 296 (1990).

[21] T. Kamae, Proceedings of the XXIV International Conference on High Energy Physics, Munich (1988), edito da R. Kotthaus e J. Kuhn, Springer-Verlag (1989), p.156.

[22] CDF Collaboration, Phys. Rev. D 44, 29 (1991).

[23] UA1 Collaboration, Phys. Lett. B 253, 503 (1991).

[24] UA2 Collaboration, Phys. Lett. B 276, 354 (1991).

[25] LEP and the LEP Electroweak Working Group Collaborations, CERN-PPE/93-157, prepared for the Europhysics Conference on High Energy Physics, Marseille", July 22-28 1993, and for the 1993 International Symposium on Lepton-Photon Interactions at High Energies, Cornell, August 10-15. 
[26] UA2 Collaboration, Phys. Lett. B 276, 354 (1992).

[27] CDF Collaboration, Phys. Rev. D 43, 2070 (1991).

[28] CDHS Collaboration, Phys. Rev. Lett. 57, 298 (1986);

CDHS Collaboration, Z. Phys. C 45, 361 (1990).

[29] CHARM Collaboration, Phys. Lett. B 177, 446 (1986);

CHARM Collaboration, Z. Phys. C 36, 611 (1987).

[30] CCFR Collaboration, M. Shaevitz, Proceedings of the La Thuile '93 Conference.

[31] UA1 Collaboration, Z. Phys. C 48, 1 (1990).

[32] UA2 Collaboration, Z. Phys. C 46, 179 (1990).

[33] CDF Collaboration, H. Grassmann, Presentation at the DESY Theory Workshop, Fermilab-Conf-92/105-E.

[34] F. Dydac, Proceedings of the 25th International Conference on High Energy Physics, Singapore 1990, edited by K.K. Phua e Y. Yamaguchi (World Scientific, Singapore 1991).

[35] CDF Collaboration, M. Cobal, Proceedings of the '99 EPS Conference, Fermilab-Conf93/320-E.

[36] R.K. Ellis, Phys. Lett. 259, 492 (1991).

[37] E. Laenen, J. Smith and W. van Neerven, Fermilab-PUB-93/270-T (1993).

[38] M. Gluck, J.F. Owens and E. Reya, Phys. Rev. D 172324 (1978);

[39] P. Nason, S. Dawson and R.K. Ellis, Nucl. Phys. B 303, 607 (1988);

B.L. Combridge, Nucl. Phys. B 151429 (1979);

K. Hagiwara e T. Yoshino, Phys. Lett. B 80282 (1979). 
[40] B. Denby, CDF internal note \# 2327

[41] CDF Collaboration, Phys. Rev. Lett. 70, 4042 (1993).

[42] CDF Collaboration, Phys. Rev. Lett. 64,147 (1990);

CDF Collaboration, Phys. Rev. D 43, 664 (1991).

[43] R. Kleiss, W.J. Stirling, Z. Phys. C 40 (1988), 419;

K. Hagivara, D. Zeppenfeld, Nucl. Phys. B 313, 560 (1989);

F.A. Berends and W.T. Giele, Nucl. Phys. B 306, 759 (1988);

W.T. Giele, Ph.D Thesis, Università di Leiden (1989).

[44] M. Valdata, XXIV International Conference on High Energy Physics, Munchen (1988).

[45] M. Cobal, H. Grassmann, S. Leone, Il Nuovo Cimento, 107 A, 75 (1994).

[46] I. Hinchliffe, 'Application of QCD to Hadron Collider Physics (Theory)', Lecture given at the SLAC Summer Institute, July 1989.

[47] F.A. Berends, W. Giele, H. Kuijf, B. Tausk, 'On the production of a W and jets at Hadron Colliders', Fermilab-Pub-90/213-T.

W. Giele, Fermilab-Pub-90/213T, Fermilab-Conf-90/229T.

[48] M. Shochet, 'The Physics of Proton Antiproton Collision', Fermilab-Conf-91/341-E.

[49] F. Abe et al. (CDF coll.), Phys. Rev. Lett. 62, 3020 (1989);

R. Harris, Proceedings of the 'Particle and Fields 91' Conference, Vancouver, Canada.

[50] H. Grassmann, Ph.D. Thesis, 'A limit on the Leptoquarks from Missing energy and from Muon Events at the $p \bar{p}$ Collider', RWTH Aachen, 1988.

[51] G. Bellettini, G. Busetto, M. Cobal, H. Grassmann, S. Leone, CDF internal note \# 1912 (1992).

[52] Nucl. Instr. Meth. A 271, 387 (1988). 
[53] "Proposal for an Upgraded CDF Detector", CDF internal note \# 1172.

[54] Nucl. Instr. Meth. A 289, 338 (1990).

[55] Nucl. Instr. Meth. A 268, 75 (1988).

[56] Nucl. Instr. Meth. A 268, 268 (1988).

[57] Nucl. Instr. Meth. A 267, 272 (1988).

[58] Nucl. Instr. Meth. A 267, 280 (1988).

[59] Nucl. Instr. Meth. A 267, 257 (1988).

[60] Nucl. Instr. Meth. A 267, 301 (1988).

[61] M.E.B Franklin et al, Proceedings of the th $^{\text {th }}$ Topical Workshop on Proton-Antiproton Collider Physics, p. 420, Fermilab, 1988, pubblicato da World Scientific.

[62] Nucl. Instr. Meth. A 267, 249 (1988).

[63] Nucl. Instr. Meth. A 268, 33 (1988).

[64] Note interne di CDF \# 1500 e 1964

[65] CDF internal note \# 1614.

[66] C.E. Pagliarone, Tesi di Laurea (1993), Università di Pisa.

[67] D. Amidei et al, Nucl. Instr. Meth. A 269, 51 (1988).

[68] S. Behrends, S. Kuhlmann, D. Amidei and F. Rimondi, CDF internal note \# 583 (1987).

[69] S. Behrends, M. Shapiro, E. Pare CDF internal note \# 1066 (1989).

[70] J. Tonnison, CDF internal note \# 2189 (1993).

[71] C. Blocker, M. Cobal, H. Grassmann, S. Leone, M. Turcotte, CDF internal note \# 2323 (1993). 
[72] D. Amidei et al., CDF internal note \# 2065 (1993).

[73] S. Leone, Ph. D. Thesis, University of Pisa (1993).

[74] CDF Collaboration, F. Abe et al., Phys. Rev. Lett. 63, 720 (1989).

[75] CDF internal note \# 1916 (1992).

[76] R. Field and R. Feynamnn, Nucl. Phys. B 136, 1 (1978).

[77] M. Cobal, H. Grassmann, S. Leone, CDF internal note \# 1533 (1991).

[78] M. Cobal, H. Grassmann, S. Leone, CDF internal note \# 1919 (1992).

[79] M. Cobal, H. Grassmann, S. Leone, CDF internal note \# 2139 (1993).

[80] J. Benlloch, A. Caner, M. Mangano, T. Rodrigo, CDF internal note \# 1823 (1992).

[81] E. Meschi, in Proceedings of the DPF Meeting of the Am. Phys. Soc., Batavia (1992), ed. C.H. Albright et al., World Sci., p. 1117.

[82] F. Paige, S.D. Protopopescu, BNL Report No. 38034 (1986).

[83] M. Shapiro, CDF internal note \# 1810 (1992).

[84] W. Giele, comunicazione privata.

[85] B. Winer, Ph.D Thesis, 'The W Boson Transverse Momentum Spectrum in ProtonAntiproton Collisions at sqrt $s=1.8 \mathrm{TeV}$, Lawrence Berkeley Laboratory, Berkeley, CA (1991).

[86] J.Ohnemus, Phys. Rev. D 44, 1403 (1991); J. Ohnemus and J.F.Owens, Phys. Rev. D 43, 3626 (1991); J.Ohnemus, FSU-HEP-910621 e Fermilab-Pub-91/163-T.

[87] M. Cobal, H. Grassmann, S. Leone, CDF internal note \# 1704 (1993).

[88] M. Cobal, H. Grassmann, S. Leone, CDF internal note \# 2162 (1993). 
Bibliography

107

[89] W. Giele, private communication.

[90] R. Hughes et al, CDF internal note \# 2068 (1993).

[91] ALEPH Collaboration, CERN-PPE/930-108 (in via di pubblicazione su Phys. Lett B); D. Amidei et al, CDF internal note \# 2091 (1993).

[92] S. Dell' Agnello, Ph.D. Thesis, Pisa (1993).

[93] C. Campagnari, P. Sphicas, A. Yagil and B. Farhat, CDF internal notes \# 1961, 2098 (1993).

[94] R. Hughes et al., CDF internal note \# 2248 (1993);

R. Hughes et al., CDF internal note \# 2343 (1993);

R. Hughes et al., CDF internal note \# 2353 (1993).

[95] M. Binkley, CDF internal note \# 2235.

[96] P. Schlabach, CDF internal note \# 2186.

[97] P. Schlabach, CDF internal note \# 2169.

[98] M. Cobal, H. Grassmann, S. Leone, CDF internal note \# 1705. 